archives-ouvertes

\title{
Imagerie expérimentale ex vivo de haute résolution à 7 tesla du cancer localisé de la prostate
}

\author{
Matthieu Durand
}

\section{To cite this version:}

Matthieu Durand. Imagerie expérimentale ex vivo de haute résolution à 7 tesla du cancer localisé de la prostate. Médecine humaine et pathologie. Université du Droit et de la Santé - Lille II, 2019.

Français. NNT : 2019LIL2S015 . tel-02464851

\section{HAL Id: tel-02464851 \\ https://tel.archives-ouvertes.fr/tel-02464851}

Submitted on 3 Feb 2020

HAL is a multi-disciplinary open access archive for the deposit and dissemination of scientific research documents, whether they are published or not. The documents may come from teaching and research institutions in France or abroad, or from public or private research centers.
L'archive ouverte pluridisciplinaire HAL, est destinée au dépôt et à la diffusion de documents scientifiques de niveau recherche, publiés ou non, émanant des établissements d'enseignement et de recherche français ou étrangers, des laboratoires publics ou privés. 
THESE DE L'UNIVERSITE DE LILLE

Délivrée par

L'UNIVERSITE LILLE 2

ECOLE DOCTORALE BIOLOGIE ET SANTE LILLE

DIPLOME DE DOCTORAT

(arrêté du 7 août 2006)

soutenue publiquement le

9 juillet 2019

par

Matthieu DURAND

TITRE :

IMAGERIE EXPÉRIMENTALE EX VIVO DE HAUTE RÉSOLUTION À 7 TESLA DU CANCER LOCALISÉ DE LA PROSTATE :

Directeur de thèse : Pr Arnauld Villers

$$
\begin{gathered}
\text { JURY : } \\
\text { Pr Jean Amiel } \\
\text { Dr Eric Barret } \\
\text { Pr Gaëlle Fromont-Hankard } \\
\text { Pr Philippe Puech } \\
\text { Dr Charles Raffaelli } \\
\text { Pr Antoine Valeri }
\end{gathered}
$$


RÉSUMÉ en français

L'IRM à 1,5T ou 3T de la prostate est l'imagerie la plus performante pour la détection du cancer de prostate. Nous avons travaillé à des modèles expérimentaux ex vivo d'IRM haute résolution à $7 \mathrm{~T}$ permettant d'améliorer la résolution spatiale et la détection tumorale.

La première partie de ce travail a porté sur la mise au point de protocoles d'analyse IRM de tissus prostatiques ex vivo à $7 \mathrm{~T}$. Des coupes de tissus de pièces opératoires et cadavériques avec et sans cancer ont été étudiées. Les données obtenues ont permis de définir des paramètres de séquence en turbo spin-echo pour obtenir des images avec résolution spatiale de $60 \times 60 \times 90 \mu \mathrm{m}^{3}$. Cette résolution permettait d'identifier à l'imagerie IRM $7 \mathrm{~T}$ des caractéristiques tissulaires comparables à celles obtenues par analyse histo-pathologique (X2).

La seconde partie de ce travail a porté sur l'étude par IRM 7T de glandes prostatiques entières chez 18 patients opérés pour cancer de prostate. En utilisant les protocoles d'acquisition mis au point dans la première étude, l'imagerie a comporté des séquences T2, à la plus haute résolution de $130 \times 130 \times 195 \mu \mathrm{m}^{3}$ et de diffusion avec cartographie $A D C$. Les résultats étaient interprétés par deux relecteurs en aveugle pour évaluer la qualité et la détection tumorale. La haute résolution spatiale des images, comparée à celle obtenue par analyse histo-pathologique, était jugée comme bonne avec une concordance inter-relecteur élevée. Une étude de corrélation anatomo-imagerie pour la détection du cancer avec analyse par secteur a montré une sensibilité de $70 \%$, une spécificité de $80 \%$, une valeur prédictive positive de $79 \%$ et une valeur prédictive négative de $72 \%$.

Ces résultats ont permis de mesurer la résolution de l'imagerie IRM à 7T ex vivo et son intérêt pour la détection du cancer. Une sémiologie IRM 7T du tissu prostatique doit être décrite. Ces résultats serviront de base à la translation future de l'imagerie actuelle vers l'IRM 7T in vivo chez l'homme. 
RÉSUMÉ en anglais

7T High resolution magnetic resonance imaging of prostate specimen: probing the radio-histological frontier

Prostate MRI at $1.5 \mathrm{~T}$ or $3 \mathrm{~T}$ is the best imaging modality for tumor detection. We investigated high resolution MRI at $7 \mathrm{~T}$ on prostate specimen tissue to improve spatial resolution and prostate cancer detection.

First part of experiments consisted of setting up new imaging protocol with 7T MRI on ex vivo prostatic tissue. Imaging was carried out on all or part of specimen from radical prostatectomy of patients or prostate harvested from deceased organ donors. Collected data resulted in new protocol parameters for fast spin echo needed to yield a spatial resolution of $60 \times 60 \times 90 \mu \mathrm{m}^{3}$. High spatial resolution imaging was used to identify relevant morphological structures for characterization of the prostate gland and tumor as compared to histology.

Second part of work was done with whole gland imaging at $7 \mathrm{~T}$ of radical prostatectomy specimens of patients. Imaging protocol was based on the outcomes from the first part of experiments and consisted in T2W with high resolution of 130 X130 X195 $\mu \mathrm{m}^{3}$, diffusion and ADC map. Two independent and blinded reviewers were in charge of imaging quality assessment and tumor detection. Overall quality was great with good agreement between the two reviewers. Correlation study for prostate cancer detection with the corresponding $\mathrm{H} \& \mathrm{E}$ was of $70 \%, 80 \%, 79 \%$ and $72 \%$ for sensitivity, specificity, positive predictive value and negative predictive value, respectively.

We measured the resolution of $7 \mathrm{~T}$ MRI of ex vivo prostatic tissue, and it's benefits in tumor detection. New semiology should be designed at $7 \mathrm{~T}$ to improve the understanding of prostatic tissue in further experiments. In future, these findings can be extrapolated to carry out in $7 \mathrm{~T} \mathrm{MRI} \mathrm{of} \mathrm{in} \mathrm{vivo} \mathrm{prostate} \mathrm{gland.}$

DISCIPLINE

Urologie

MOTS-CLES

Cancer de prostate, Diagnostic, Imagerie, Résonance magnétique nucléaire

INTITULÉ ET ADRESSE DE L'U.F.R. OU DU LABORATOIRE :

INSERM, U1189, ONCO-THAI, Dr S MORDON, Lille, France 


\section{Table des matières}

I. Introduction

II. Contexte.

- Épidémiologie du cancer de prostate

- Définition d'une lésion significative

- Stratégie de dépistage du cancer de prostate recommandée

- Mise à jour \& limites des indications actuelles d'IRM prostatique

- Principes physiques de la résonance magnétique nucléaire

- Application en imagerie médicale : séquences \& contraste

- Optimisation des séquences

III. Enjeux \& Objectifs

- Enjeux de recherche

- Premier objectif

- Deuxième objectif

IV. Modèle \& Travaux expérimentaux 1

- Objectif principal

- Cohorte d'étude

- Cadre réglementaire de déclaration d'étude

- Consentements patients \& risques

- Préparation, manipulations \& suivi des échantillons prostatiques pour imagerie

- Imagerie des glandes entières ex vivo

- Imagerie des coupes de tissus ex vivo

- Critères de jugement et mesure des objectifs

- Protocoles d'imagerie

- IRM 3T haute résolution

- IRM 7T haute résolution

- Analyse anatomo-pathologique et étude de corrélation

- Résultats

- Discussion

V. Modèle \& Travaux expérimentaux 2.

- Objectifs principal \& secondaires

- Cohorte d'étude

- Consentement $\&$ cadre réglementaire

- Préparation, manipulations \& suivi des échantillons prostatiques pour imagerie

- Protocoles d'imagerie IRM 7T haute résolution

- Analyses de l'imagerie

- Analyse morphologique qualitative de l'imagerie

- Analyse de susceptibilité lésionnelle tumorale

- Analyse descriptive de l'imagerie des lésions confirmées à l'histologie

- Analyses histologiques

- Analyses morphométriques de comparaison anatomo-clinique : corrélation

- Analyses statistiques

- Résultats

- Discussion

VI. Perspectives de développement et d'applications cliniques

VII. Conclusions

VIII. Références.

IX. Articles publiés dans le cadre de la thèse. 


\section{Introduction}

L'imagerie par résonnance magnétique nucléaire (IRM) dans le cancer de la prostate connaît un essor considérable. Elle est considérée comme la technique d'imagerie non-invasive la plus prometteuse pour la détection et la caractérisation des lésions cancéreuses prostatiques [1,2] et pour l'identification des profils de patients à haut risque [3-6]. On dispose désormais d'un haut niveau de preuve pour démontrer de son bénéfice dans la prise en charge des patients suspects de cancer de prostate présentant une élévation de PSA. Pour autant, même si sa réalisation est récemment recommandée par l'AFU avant de pratiquer des biopsies prostatiques [7], l'ensemble de ses indications fait toujours l'objet de débats continus et varie de par le monde [1] par défaut de performance.

En pratique, I'IRM de prostate obtient le meilleur taux de détection de cancer significatif (volume $>0,5 \mathrm{~cm}^{3}$ ) grâce à la combinaison multiparamétrique de séquences d'imagerie anatomique T2 (T2 Weighted), d'imagerie de diffusion pondérée (DWI Diffusion Weighted Imaging) et d'imagerie T1 avec injection de contraste (DCE Dynamic Contrast-Enhanced) [8,9]. Mais bien que I'IRM soit considérée comme l'imagerie la plus adaptée, ses performances sont telles qu'aujourd'hui, elle ne peut être recommandée comme un test diagnostique autonome, à la place des biopsies de prostate, du fait du risque rapporté dans les meilleures équipes de manquer $13 \%$ des patients qui présentent un cancer significatif avec un score de Gleason >= 7 [10].

En effet, l'IRM n'offre pas, dans sa conformation clinique actuelle, assez de résolution spatiale pour établir une corrélation radio-histologique fiable et suffisante pour traduire 
en anomalie de signal chaque lésion anatomopathologique existante. Ce manque de puissance limite ses capacités diagnostiques. Son déficit de résolution restreint son pouvoir discriminant d'identification lésionnelle. II réduit donc son recours dans la prise de décisions cliniques éclairées pour apporter suffisamment d'assurance dans des propositions de programmes personnalisés de soins de type surveillance active, thérapie focale ou certaines techniques de préservation neuro-vasculaire. Concrètement, si l'IRM est capable de détecter une anomalie associée à la présence d'un cancer de prostate invasif et volumineux, sa résolution spatiale la limite pour caractériser des lésions au niveau microscopique, qui est pourtant essentiel pour permettre des détections lésionnelles précoces, la mesure précise des extensions carcinologiques et l'appréciation de leur agressivité.

Plusieurs leviers de performance ont déjà été étudiés. Notamment, l'optimisation de séquences IRM grâce à l'usage de champs magnétiques plus élevés pour apporter en théorie plus de signal et de contraste. Des recherches ont été conduites dans ce sens sur le gain potentiel mesuré par l'usage de champs magnétiques plus élevés de 1,5 à 3 Tesla (T) [8] dans le but d'améliorer la résolution et le contraste en IRM clinique. Les résultats n'ont cependant pas toujours su démontrer de supériorité significative [11]. De ce constat, nous avons souhaité vérifier l'hypothèse que l'augmentation de la résolution spatiale, au-delà de celle obtenue sur des IRM cliniques actuelles, pourrait bien permettre d'apporter un gain de niveau de signal et de contraste suffisant pour détecter des anomalies tissulaires à l'échelle microscopique.

Considérant l'ensemble des indications cliniques actuellement recommandées par les sociétés savantes pour l'IRM de prostate, et basant notre réflexion sur les principes 
physiques de la résonnance magnétique nucléaire pour en comprendre les voies d'amélioration, nous avons exécuté différentes séries de tests. Plusieurs protocoles sur IRM expérimentale ont été utilisés afin de rechercher les paramètres de séquences nécessaires à l'établissement d'une résolution spatiale satisfaisante pour permettre une corrélation fiable entre anomalie de signal et lésion histologique architecturale micro-tissulaire. Notre exposé présente consécutivement les résultats de deux séries de travaux réalisés sur plusieurs années dans les laboratoires d'imagerie, d'anatomopathologie, et de chirurgie et de Weill Cornell Medical College (WCMC) et Mount Sinai Hospital à New-York, sous la direction opérationnelle de Pr AK Tewari \& la supervision de Pr Arnauld Villers sous l'égide de l'Unité Inserm U1189, ONCO-THAI, de Dr S. Mordon. ${ }^{1}$

\footnotetext{
${ }^{1}$ A noter que le Score de Gleason a été redéfini depuis par l'ISUP en 5 groupes pronostiques. Le travail réalisé ayant été antérieur, la classification par Score de Gleason a été conservée.
} 


\section{Contexte}

\section{a. Épidémiologie du cancer de prostate}

Le cancer de la prostate est le $2^{\text {ème }}$ cancer le plus fréquent de l'homme en France après les tumeurs cutanées. On rapporte une sensible diminution de son taux d'incidence, actuellement mesuré autour de 50000 nouveaux cas par an (97,7/100 000 ; - 6\% par an) [12]. L’âge médian au diagnostic se situe juste avant 70 ans. Deux facteurs de risques sont identifiés, l'origine afro-américaines et les antécédents familiaux. L'origine familiale fait l'objet de recherches soutenues en raison de son importance puisque $22 \%$ des patients rapportent avoir un père ou un frère atteint de cancer de prostate. L'identification de gènes impliqués dans la tumorigenèse pour contribuer au dépistage de cette maladie est une voie de recherche importante. Une étude canadienne récente sur 1858 patients atteints d'un cancer agressif, rapporte notamment l'existence d'une mutation du gène Kallikrein sur le chromosome 19 dans 6 à $14 \%$ des cas. D'autres études soulignent la potentielle implication de la mutation BRAC2 associée à des cancers de pronostics plus sévères.

On décrit des formes cliniques très variées du cancer de la prostate dont toutes n'engagent pas le pronostic vital. Il est aujourd'hui la $3^{\text {ème }}$ cause de décès par cancer chez l'homme en France (8 207 cas estimés en 2017) avec un âge médian au moment du décès de 83 ans. Globalement, on enregistre une tendance à la baisse de la mortalité (de 18/100 000 en 1990 à 10,5/100 000 en 2011, -4\% par an). Son taux de survie nette à 5 ans est de plus de $93 \%$ et $80 \%$ à 10 ans, tout stade confondu. Ces résultats encourageants s'expliquent en partie par l'amélioration des traitements, notamment à la phase métastatique, la plus sévère, où la survie globale a été 
multipliée par 3 en quelques années, avec, actuellement, une médiane à 32,5 mois [13], depuis notamment l'instauration des hormonothérapies de nouvelle génération. Le recours au test de dépistage par dosage de PSA et l'instauration du schéma de biopsies systématisées étendues comme test diagnostique de référence concourent aussi à la baisse de l'incidence de la mortalité par l'augmentation diagnostique d'une majorité de tumeurs de stades précoces intra-prostatiques [14].

\section{b. Définition d'une lésion significative}

La détection précoce de cancers de prostate asymptomatiques a conduit à l'augmentation de l'incidence de lésions de faible volume bien différenciées (environ $30 \%$ ) à faible risque de développement symptomatique agressif au cours de la vie, dites lésions indolentes [15]. Cette notion comporte, en réalité, plusieurs définitions. Stamey et al a été le premier à la préciser, considérant comme non significatif un cancer $<0,5$ cc de volume sans présence de grade 4 ou 5 , sur la base d'une étude de clinique de 139 spécimens de cystoprostatectomies radicales [16]. La définition a, par la suite, évolué avec Epstein qui a proposé un volume seuil de 0,2 cc, du fait de l'existence de pT3a rapporté sur des tumeurs entre 0,2 et 0,5 cc [17]. Un modèle prédictif de cancer indolent a alors été proposé sur cette base de définition, en corrélant les résultats de biopsies de patients et les données anatomo-pathologiques finales après prostatectomie radicales : <=T1c, densité de $\mathrm{PSA}<0,15,<=2$ biopsies positives avec <= 50\% d'envahissement des carottes sans grade 4 ou 5 de Gleason. Pour l'heure cette norme fait référence dans la plupart des cas bien que plusieurs études ont rapporté un risque d'erreur diagnostique de manquer des lésions significatives en se limitant au modèle prédictif d'Epstein [18]. De nombreuses 
définitions cohabitent donc aujourd'hui dont certaines intègrent les résultats d'imagerie IRM pour mieux apprécier le volume et le grade tumoral en plus des autres indicateurs.

\section{c. Stratégies dépistage de cancer de prostate recommandées}

À l'heure actuelle, il n'est recommandé par aucune autorité compétente de procéder à un dépistage systématique du cancer de la prostate en France ou dans le reste du monde car aucune étude épidémiologique n'a apporté la preuve d'un bénéfice en termes de survie [19-21].

Des faisceaux de preuves abondent même en faveur d'un risque de sur-diagnostic et de sur-traitement, renforçant l'importance de bien distinguer les formes agressives des plus indolentes pour adapter les projets thérapeutiques en conséquence.

Selon l'AFU ou l'EAU, le dépistage doit demeurer individuel et volontaire et, en l'absence de facteur de risque, proposé pour les patients de 50 à 75 ans. Il est réalisé au moyen d'un examen clinique par toucher rectal et d'un dosage du taux de PSA sérique. Et d'après le récent Congrès européen d'urologie (EAU) de 2018, les recommandations pourraient connaître une prochaine évolution avec l'avènement de l'IRM et de tests génétiques en complément du dosage de PSA.

En faveur de l'apport de l'IRM, une étude britannique récente (PROMIS) [22] a enrôlé 740 hommes. Ils étaient tous soumis à un test de PSA, une biopsie et une IRM multiparamétrique. L'apport de l'imagerie s'est révélé supérieur à celui de la biopsie pour détecter les cancers de prostate, avec moins d'effets secondaires. D'autres études devront cependant être réalisées avant d'envisager un dépistage par IRM, sachant qu'il faudrait aussi pour changer les règles considérer les problèmes de coût 
et de disparité d'expertises qui restent des freins à la généralisation de l'IRM prostatique dans le screening de la maladie [23].

A noter que la Haute Autorité de Santé (HAS) a adopté une position plus radicale inverse depuis mars 2015, qui n'est partagée d'aucune société savante d'urologie, ni française ni internationale ou européenne. A savoir, elle précise qu'il n'a pas été retrouvé d'éléments scientifiques suffisants permettant de justifier un dépistage, même individuel, par dosage de PSA, y compris pour les sous-populations considérées comme plus à risque. La controverse demeure sur ce point.

\section{d. Mise à jour \& limites des indications actuelles d'IRM prostatique}

L'analyse de l'ensemble des indications IRM a été réalisée suite à une revue de la littérature portant sur les données récentes de performances diagnostiques IRM et leur impact sur la prise en charge du cancer de la prostate [11].

Ce travail de mise à jour a été commandé par the International Consultation on Urological Diseases ICUD de la Société Internationale d'Urologie (SIU) qui a nommé un comité chargé d'examiner la littérature sur l'IRM de prostate. Dans ce cadre, nous avons mené une recherche dans la base de données Pub Med qui a permis d'identifier les articles sur les protocoles de détection et de stadification en IRM multiparamétrique, les systèmes de notification et de notation, le rôle de l'IRM dans le diagnostic de cancer de prostate avant biopsie, pour la surveillance active, la thérapie focale, la détection locale et la récidive après traitement.

Des divergences d'opinion ont été rapportées dans l'utilisation de la force des aimants [1,5 Tesla $(T)$ vs. $3 T$ ] et des bobines sans consensus. Plus d'accord a été trouvé concernant le choix des séquences d'impulsions. Aucune séquence ne suffisant à elle- 
même, il est recommandé d'associer: IRM pondérée en diffusion (DWI) avec cartographie ADC, IRM dynamique avec perfusion de Gadolinium (DCE-IRM) et / ou IRM spectroscopique (ISRM) - rarement réalisée dans les faits - en plus des séquences anatomiques pondérées en T2.

En 2015, le système de reporting et de données d'imagerie de la prostate (PI-RADS version 2) a été développé pour standardiser l'acquisition, l'interprétation lésionnelle des images [24] \& l'agressivité tumorale (PIRADS 2) [25]. La communication des résultats devrait reposer désormais sur une représentation cartographique précise de la glande, partagée en 27 secteurs, pour aider à uniformiser les informations sur la localisation des détections comme décrit déjà antérieurement [26] (Figure 1). 


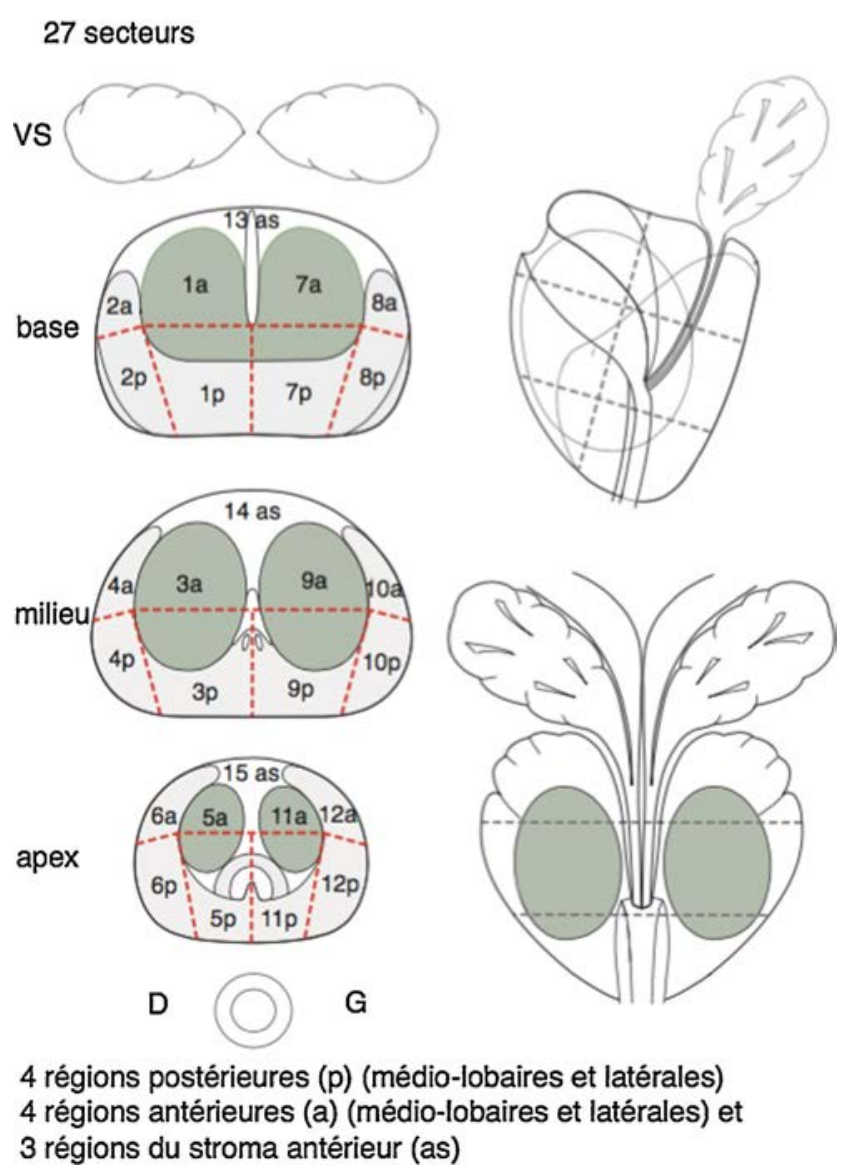

Figure 1 - Schéma de prostate en 27 secteurs pour l'interprétation standardisée de l'IRM et la réalisation des biopsies ciblées [26]

En termes de résultat, I'IRM multiparamétrique améliore la détection du cancer de prostate cliniquement significatif en cas de biopsies répétées ou avant la biopsie de confirmation chez les patients envisageant une surveillance active. Elle est utile pour guider le traitement focal et pour détecter les récidives locales après le traitement. Son rôle au cours de la surveillance active reste discuté. L'ensemble des indications est présenté dans le Tableau 1.

Les performances actuelles de l'IRM en pratique clinique sont variables et permettent de détecter des lésions cancéreuses à partir de $0,2 \mathrm{cc}$ et $7 \mathrm{~mm}$ de diamètre [27] avec une sensibilité (Se) qui oscille entre $55 \%$ et $88 \%$ pour une spécificité (Sp) qui varie de $67 \%$ à $82 \%$ [28]. Les performances sont accrues en cas de tumeur localement 
avancées avec extension extra-prostatique du cancer, Se 86\%, Sp 81\% (extension envahissante par exemple, les bandelettes neuro-vasculaires, le sphincter urétral, les vésicules séminales...) [29].

Tableau 1 - Synthèse de l'ensemble des indications d'utilisation de l'IRM en pratique Clinique courante

\begin{tabular}{|c|c|c|}
\hline Indications / intérêt de I'IRM & Commentaires & Références \\
\hline Identification des cancers significatifs & $\begin{array}{l}\text { - pour des lésions }>0,5 \mathrm{cc} \\
\text { - en particulier les tumeurs antérieures }\end{array}$ & \\
\hline Staging lésionnel & $\begin{array}{l}\text { - localisation et extension précise, rapport } \\
\text { anatomique avec les autres structures } \\
\text { (urètre, capsule, col vésical, vésicules } \\
\text { séminales, rectum), extension extra- } \\
\text { prostatique }\end{array}$ & \\
\hline Évaluation de l'agressivité & $\begin{array}{l}\text { - corrélation inversement proportionnelle } \\
\text { entre le score ADC et le SG, plus l'ADC } \\
\text { est bas plus le SG est élevé }\end{array}$ & $\begin{array}{l}\text { T. Kobus et al, } 2011[30] \\
\text { Hambrock T, et al } 2011 \text { [31] }\end{array}$ \\
\hline Bilan loco-régional & $\begin{array}{l}\text { - analyse des aires ganglionnaires } \\
\text { iliaques et combo-aortique, suspicions si } \\
\text { taille iliaque }(>8 \mathrm{~mm}) \text { lombo-aortique } \\
(>10 \mathrm{~mm}) \text { et si forme arrondie plutôt } \\
\text { qu'ovalaire }\end{array}$ & JC Weinreb et al, 2016 [25] \\
\hline $\begin{array}{l}\text { Assistance aux biopsies ciblées cognitive } \\
\text { ou par fusion IRM/écho pour détection } \\
\text { précoce de cancer }\end{array}$ & $\begin{array}{l}\text { - augmentation de la rentabilité des } \\
\text { biopsies par augmentation du taux de } \\
\text { détection de cancer significatif } \\
\text { - taux de détection de } 50 \% \text { après } 1 \text { ou } 2 \\
\text { séries négatives Vs } 20 \% \text { en cas de re- } \\
\text { biopsie écho-guidée classique }\end{array}$ & CM Moore et al, 2013 [32] \\
\hline Éviter les biopsies inutiles & $\begin{array}{l}\text { - disparition des recommandations des } \\
\text { biopsies en saturation en cas d'IRM } \\
\text { négatif }\end{array}$ & $\begin{array}{l}\text { S. Guichard et al, } 2007 \text { [33] } \\
\text { MR Pokomy et al, } 2014 \text { [34] }\end{array}$ \\
\hline $\begin{array}{l}\text { Pré-planning de traitement de thérapie } \\
\text { focale }\end{array}$ & $\begin{array}{l}\text { - identification de la lésion index et des } \\
\text { lésions secondaires }\end{array}$ & $\begin{array}{l}\text { HU Ahmed et al, } 2011[35] \\
\text { IA Donaldson et al } 2015[36]\end{array}$ \\
\hline Monitoring des thérapies focales & $\begin{array}{l}\text { - suivi post-traitement de la cible traité et } \\
\text { surveillance secteur non traité }\end{array}$ & S. Marshall et al, 2015 [37] \\
\hline $\begin{array}{l}\text { Surveillance active \& et surveillance } \\
\text { après traitement primitif localisé }\end{array}$ & $\begin{array}{l}\text { - Identifier les évolutions entre les IRM de } \\
\text { contrôle \& détecter la récidive locale ou } \\
\text { loco-régionale précoce }\end{array}$ & $\begin{array}{c}\text { F. Marlière et al, } 2014 \text { [38] } \\
\text { R.C.N. van den Bergh et al, 2014 [39] } \\
\text { Ouzzane et al, } 2015 \text { [41] }\end{array}$ \\
\hline
\end{tabular}

Malgré les améliorations spectaculaires de ses résultats, l'IRM de la prostate demeure limitée dans sa capacité diagnostique, réservant encore son recours systématique en situation clinique. Notamment, son manque de puissance diagnostique mise en évidence par un échec de détection de cancer significatif dans $13 \%$ des cas [10] n'a pu être dépassé. Même avec l'effort de standardisation, l'hétérogénéité d'usage en pratique, le caractère relecteur-dépendant, les artefacts (prothèse, hémorragie prostatique...) et la courbe d'apprentissage longue ralentissent son expansion et la 
systématisation de cet examen. Ce constat fait naître des voies d'améliorations importantes qui sont à la base de la réflexion qui a conduit notre démarche vers une recherche d'optimisation de séquences pour plus de performance.

\section{e. Principes physiques de la résonance magnétique nucléaire}

La base de nos travaux de recherche repose sur la compréhension des principes physiques de la résonance magnétique nucléaire. Ces notions constituent un prérequis indispensable à assimiler pour comprendre les paramètres de séquences IRM modifiables permettant d'obtenir les améliorations de résolution envisagées.

\section{i. Le moment magnétique}

Le noyau de l'atome est constitué de neutrons, qui ne comportent aucune charge électrique, et de protons, chargés positivement. Ce noyau est animé d'un mouvement de rotation sur lui-même. Il est assimilé à une sphère tournant autour de son axe. Le mouvement circulaire de ses charges induit un champ magnétique qui s'appelle le moment magnétique nucléaire $(\mu)$. II s'agit d'une grandeur vectorielle qui permet de caractériser l'intensité d'une source magnétique. Cette source peut être un objet aimanté ou un courant électrique. L'aimantation qui en résulte correspond à la distribution spatiale de ce moment magnétique. En synthèse, le noyau peut être considéré comme une barre magnétique aimantée.

En l'absence de champ magnétique extérieur, tous les atomes qui ont un vecteur moment magnétique sont orientés dans des directions et des sens totalement aléatoires (Figure 2). 
En cas de champs magnétique extérieur non nul (B0), les atomes vont alors tous avoir un vecteur moment magnétique orienté dans la même direction que ce champ magnétique extérieur sans nécessairement être dans le même sens.

Les moments magnétiques en état de spin-up (noyaux parallèles) vont être orientés dans le même sens du champs magnétique B0 alors que les noyaux en état de spindown (noyaux anti-parallèles) vont aller dans l'autre sens.

A l'état de base, il y a plus de noyaux en spin-up étant donné que cet état est d'énergie inférieure - état d'énergie fondamentale dans lequel les noyaux sont beaucoup plus stables -. Les noyaux en spin-down ont besoin d'énergie pour être dans cet état excité et sont donc, par conséquence, moins nombreux.
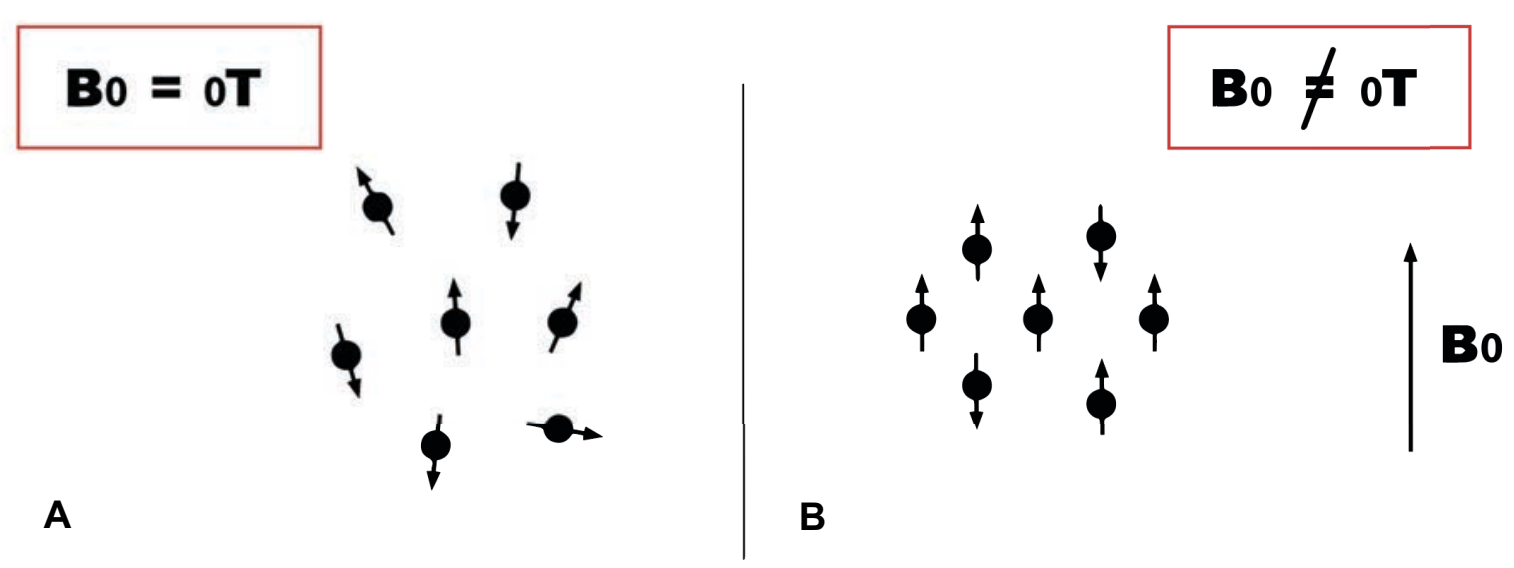

B

\section{Figure 2 - Effet d'un champ magnétique imposé sur les noyaux}

En l'absence de champ magnétique extérieur $(A)$, tous les noyaux des atomes ont un vecteur moment magnétique dans des directions et des sens totalement aléatoires. En cas d'application d'un champ magnétique non nul imposé B0 (B), les vecteurs moment magnétique des noyaux vont adopter la même direction que le champ B0, mais pas nécessairement dans le même sens. Les moments magnétiques spin-up vont être orientés dans le sens de B0 ; les spin-down dans l'autre. 


\section{ii. La résonance $\&$ aimantation}

En réalité, en présence d'un champs magnétique extérieur B0, les noyaux vont s'orienter dans la direction de ce champ avec une légère déviation d'un angle $\alpha$ autour de l'axe $z$ du champ magnétique B0. Cela est dû au moment cinétique du noyau qui va lui permettre de tourner sur lui-même. Le noyau tourne donc sur lui-même selon un mouvement de précession tout en tournant autour de l'axe z selon une certaine fréquence appelée, fréquence de Larmor. La vitesse de rotation autour de l'axe z est très variable et fonction de la nature des noyaux. Cette fréquence de Larmor dépend du rapport géomagnétique, différent pour chacun des noyaux.

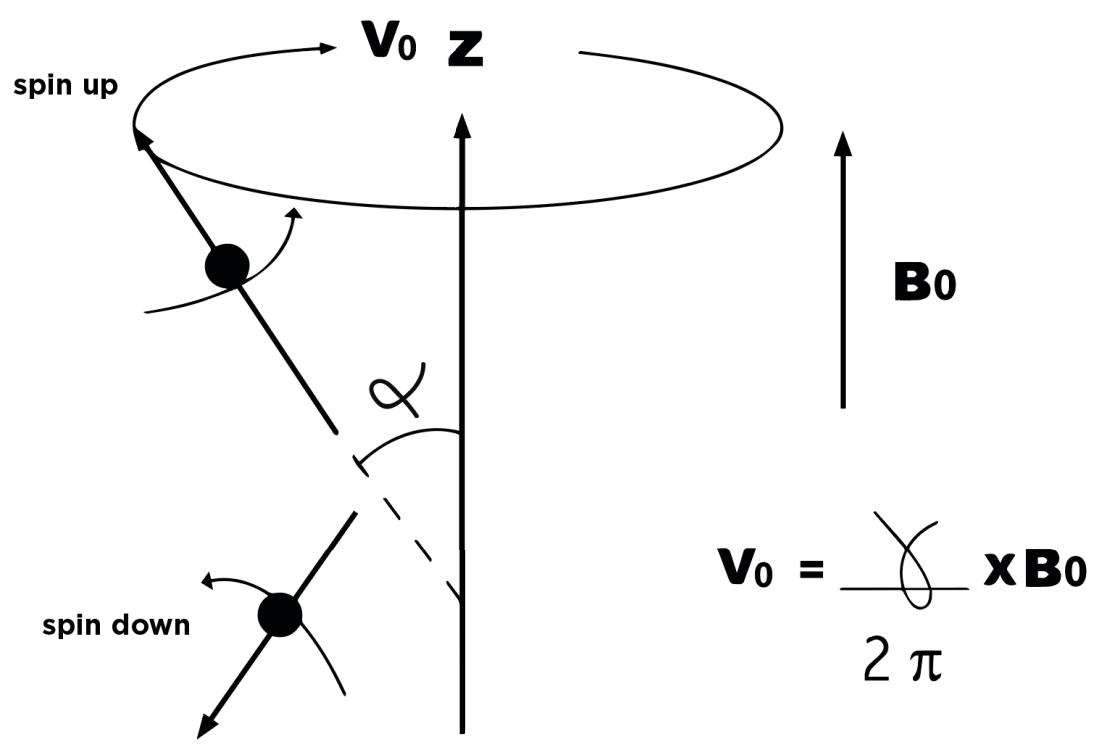

Figure 3 - Précession de Larmor

En présence d'un champ magnétique extérieur, les noyaux vont prendre la même direction avec une certain déviation selon un angle $\alpha$, dû au moment cinétique du noyau. Le noyau tourne donc sur lui-même et aussi autour de l'axe du champ magnétique selon une certaine fréquence dite de Larmor $\left(V_{0}\right)$ différente pour chaque noyau. Cette fréquence, représentée par la pulsation $\left(V_{0}\right)$ dépend du rapport gyromagnétique (f) qui correspond au rapport entre le moment magnétique $(\gamma)$ et le moment cinétique d'une particule, soit $f=\gamma / 2 \pi$, multiplié par l'intensité du champ magnétique extérieur B0 auquel est soumis l'atome. 
L'aimantation est proportionnelle au nombre de noyaux et c'est elle qui est mesurée en résonance magnétique nucléaire $(\mathrm{RMN})$. Toutefois, l'aimantation n'est pas observable lorsqu'elle est parallèle à B0. II faut donc la basculer de $90^{\circ}$.

Pour la faire basculer, il faut appliquer transitoirement un autre champ magnétique, $\mathrm{B} 1$, orienté à $90^{\circ}$ de $\mathrm{B} 0$. Sous l'effet du champ B1, la majorité noyaux en spin-up, initialement à l'état fondamental, vont gagner en énergie et passer en spin-down.

Soumis initialement au champ statique B0, l'aimantation totale suit uniquement l'axe $\mathrm{z}$, avec une aimantation longitudinale (Mz) maximale. En présence du champ B1, l'aimantation totale va donc basculer en continuant de tourner. Elle perd alors en aimantation longitudinale et gagne en aimantation transversale (Mxy) (Figure 4).

A noter qu'il faut que la fréquence du champ B1 envoyé soit égale à la fréquence de Larmor afin d'être efficace. Cela permet ainsi de faire appliquer le champ B1 au moment où il peut taper l'aimantation pour la faire basculer, sans quoi, l'effet serait nul.

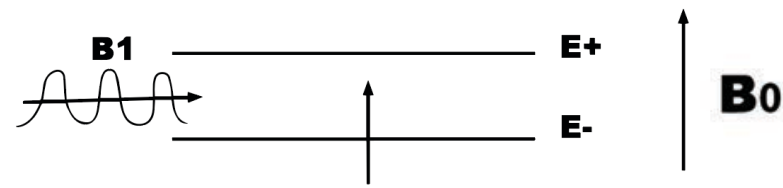

A

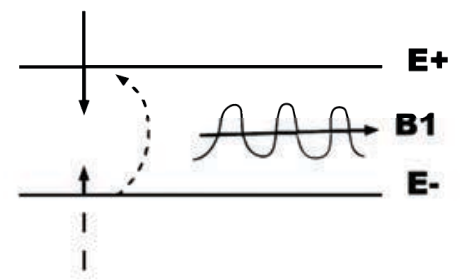

Bo

B

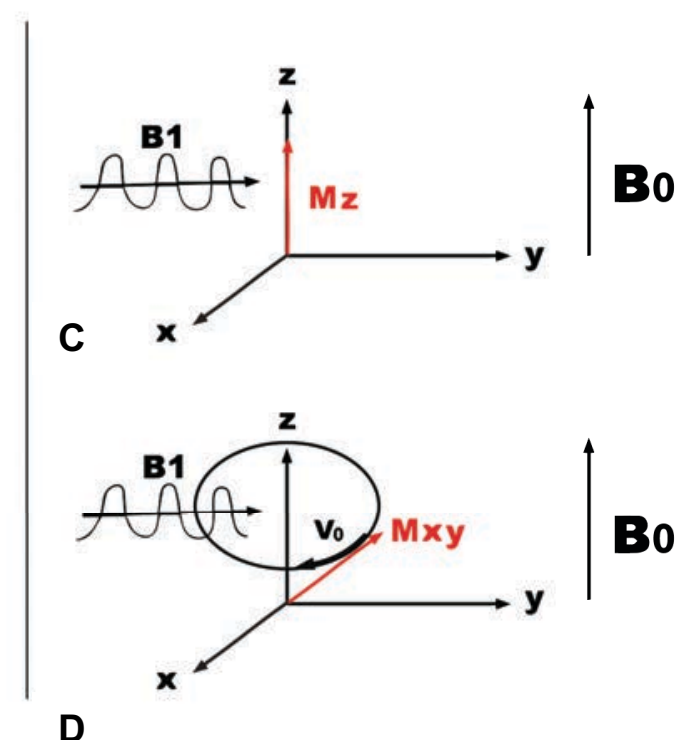

Figure 4 - Effet sur l'aimantation

En cas d'application d'un champ de radiofréquence $\mathrm{B} 1$ à $90^{\circ}$ de $\mathrm{B} 0(\mathrm{~A})$, les noyaux des atomes initialement majoritairement en spin-up vont être excités et passer en d'énergie supérieure, en spin-down (B). En termes d'aimantation, sous l'effet de B0 uniquement, on a une aimantation totale longitudinale maximale suivant l'axe $\mathrm{z}$ (C). Avec l'apport du champ de radiofréquence B1, l'aimantation totale va basculer en continuant de tourner autour de l'axe $z(D)$ et perdre ainsi en aimantation longitudinale pour gagner en aimantation transversale. 


\section{iii. La relaxation \& la RMN}

Pour générer de l'énergie, dès qu'une rotation de $90^{\circ}$ est obtenue, le champ B1 est arrêté. Tous les spins qui étaient passés à l'état down vont alors revenir à l'état up non excité. Sur le plan de l'aimantation, cela va se traduire par le phénomène inverse à celui précédemment décrit. L'aimantation qui avait basculé en transversale va donc progressivement retrouver une aimantation longitudinale maximale, sur sa position d'équilibre parallèle à B0.

Cette aimantation va prendre un certain temps pour revenir suivant l'axe $z$. Ce temps va pouvoir alors être mesuré grâce à une antenne détectrice ; on l'appelle le temps de relaxation.

À un instant quelconque d'une expérience de RMN, l'aimantation a toujours à la fois une composante perpendiculaire à $\mathrm{B} 0$, soit l'aimantation transversale, et une composante longitudinale. Ces 2 composantes sont affectées différemment par la relaxation. Le retour de l'aimantation longitudinale vers la valeur d'équilibre M0 se passe en règle générale selon un processus exponentiel.

Le temps caractéristique de la croissance de l'aimantation longitudinale est appelé temps de relaxation longitudinale et noté $\mathrm{T} 1$. De la même manière, l'aimantation transversale décroît le plus souvent de façon mono-exponentielle. Le temps caractéristique de la croissance de l'aimantation transversale est appelé temps de relaxation transversale et noté T2. T2 est toujours inférieur à T1.

Ce temps de relaxation va être caractéristique pour les tissus traversés et différent pour l'os, les liquides, la graisse... etc. Précisément, les tissus denses mettent plus de temps à se réaligner que les tissus mous. Dans les liquides, les $\mathrm{T} 1$ des protons sont typiquement très rapides et de l'ordre de quelques centaines de millisecondes à 
quelques secondes. La valeur du T2, elle, est extrêmement variable. Dans les solides, le T2 peut être plus court que la milliseconde alors que dans les liquides, il peut être plus proche du T1. Ainsi, grâce à la mesure du temps de relaxation, on peut créer des contrastes en IRM et arriver à identifier la nature des tissus traversés.

\section{f. Application en imagerie médicale : séquences $\&$ contraste}

En imagerie médicale, l'application de l'ensemble des principes physiques de RMN permet la réalisation d'un examen IRM. L'appareil est constitué d'un aimant de grande puissance dans lequel est placé le patient. La technique consiste à appliquer un premier champ B0 puis un second B1 pour modifier l'aimantation longitudinale maximale en transversale. Comme expliqué précédemment, l'arrêt du champ B1 va progressivement faire revenir l'aimantation dans un axe $z$ suivant un certain temps de relaxation mesuré par les antennes détectrice à l'origine de la création de contraste, observé sur écran, qui diffère en fonction de la nature des éléments organiques traversés.

En imagerie prostatique, l'antenne est disposée sur le pelvis du patient ou, plus rarement aujourd'hui dans le rectum au moyen d'une sonde. Le patient est allongé en décubitus dorsal sur un plan horizontal qui a été translaté dans le tunnel de l'aimant pour permettre l'acquisition. L'examen dure généralement de 15 à 20 minutes en pratique clinique courante et se composent de différentes séquences d'acquisition qui constituent l'examen IRM de référence.

Une séquence IRM correspond à l'ensemble des paramètres définissant les impulsions de champ magnétique et les caractéristiques des mesures effectuées. La 
séquence se caractérise par deux principaux paramètres modulables : le temps d'écho (TE) qui correspond à la durée entre le sommet de l'impulsion d'excitation et la moitié du temps de lecture ; et le temps de répétition (TR) qui correspond à la durée au bout de laquelle on reproduit la séquence.

En pratique, c'est dans le choix du temps de répétition et du temps d'écho que l'on influence le contraste de l'image, c'est à dire la différence d'intensité d'un signal entre 2 tissus qui sera maximum dans un intervalle de temps judicieusement choisi. On dit pondérer l'image en T1, en T2 ou en densité de proton (DP). Ce sont sur ces paramètres que nos expérimentations ont concentré leurs tests et analyses.

Le temps de répétition va permettre d'agir sur le niveau de repousse du signal. Au temps d'écho, on mesure un signal lors de sa décroissance en T2. Pour un temps de répétition court par rapport au $\mathrm{T} 1$ du tissu, la valeur de repousse du vecteur d'aimantation longitudinale en T1 est interrompue avant qu'il n'ait le temps de retrouver sa valeur d'équilibre. A l'inverse, pour un temps de répétition long, le vecteur d'aimantation longitudinale repousse à sa valeur d'équilibre. Le temps de répétition conditionne en fait la repousse du vecteur d'aimantation longitudinale.

La mesure de l'aimantation ne peut se réaliser que dans le plan transversal. Pour se faire, il faut basculer le vecteur d'aimantation par une impulsion de $90^{\circ}$ dans le plan transversal. La mesure est réalisée sur la courbe de décroissance en T2, au temps d'écho. Un temps d'écho court ne laissera pas au spin le temps de se déphaser en T2. Le signal enregistré ne sera pas représentatif de la décroissance en T2. Après un temps d'écho long, le déphasage dû à l'environnement moléculaire sera maximum. Le 
signal enregistré sera représentatif du temps de déphasage des protons, c'est-à-dire de la composante transversale de l'aimantation.

Lorsque deux tissus ont des T1 différents, c'est la vitesse de repousse de l'aimantation qui va permettre de les différencier et donc le temps de répétition influencera le contraste en T1. Si l'on choisit un temps de répétition long, la repousse sera complète entre les deux tissus et le signal ne permettra pas de les différencier par rapport à leur T1. Par contre, si l'on fait le choix d'un temps de répétition court, le tissu possédant un T1 court aura un niveau de repousse supérieur donc un signal plus élevé par rapport à celui qui possède un T1 long de signal plus faible.

Pour rendre compte du niveau de repousse de l'aimantation longitudinale, il faut appliquer d'abord une impulsion de $90^{\circ}$, puis une impulsion de $180^{\circ}$, pour mesurer le signal au temps d'écho. Lorsque le temps d'écho est court, le déphasage intervient le moins possible. Dans une séquence pondérée en $\mathrm{T} 1$, le temps de répétition utilisé est court pour que la différence de repousse des aimantations longitudinale soit maximale ; le temps d'écho est court pour que le déphasage des spins, c'est-à-dire l'influence du T2, soit minimale.

Lorsque deux tissus ont des T2 différents. C'est le déphasage lié à l'environnement moléculaire qui est à l'origine du contraste en T2. II faut donc utiliser un temps d'écho suffisamment long pour que la différence des signaux puisse s'exprimer. Ainsi, pour différencier 2 tissus, sur la base de leur T2 - situation caractéristique de notre modèle d'étude en imagerie prostatique -, on attend un temps de répétition long qui permet de s'affranchir des différences en T1, puis un temps d'écho long qui permette aux 
différences de signal en T2 de s'exprimer. L'image produite par une séquence pondérée en T2 montre un signal maximum pour les zones dont les protons se déphasent le plus lentement et un signal minimum pour les zones où les protons se déphasent rapidement.

A cela se rajoute le choix du temps de répétition en fonction de la densité protonique. II s'agit là du troisième paramètre accessible pour mettre en évidence un tissu. La taille ou module du vecteur d'aimantation longitudinale à l'équilibre est directement proportionnel à la quantité de proton présent dans le tissu considéré. L'estimation de la hauteur du vecteur d'aimantation longitudinale va donc permettre de différencier les tissus sur la base de leur différence en densité protonique.

Hors modèle expérimentale, en pratique clinique courante, la séquence IRM la plus fréquente est sans doute la séquence écho de spin qui se compose ainsi :

- $\quad$ une impulsion à $90^{\circ}$ d'excitation ;

- $\quad$ une période de déphasage dans le plan transverse des protons pendant le temps d'écho/2 ;

- $\quad$ une impulsion à $180^{\circ}$, dite d'inversion ;

- $\quad$ un rephasage pendant le temps d'écho / 2 ;

- $\quad$ une lecture finale du signal (= lecture de l'écho de spin).

\section{g. Optimisation des séquences}

L'optimisation des séquences résulte d'un compromis entre le contraste, le bruit et la résolution. II est indispensable de maîtriser ces notions pour permettre de comprendre les modèles expérimentaux que nous avons suivis. 


\section{i. Le contraste}

Le contraste de l'image est lié à des facteurs propres aux tissus (T1, T2, DP...) et aux paramètres de la séquence IRM utilisée (TR, TE...). Notre travail a été guidé par des mesures d'optimisation de contraste qui découlent de notre analyse présentée dans le paragraphe précédent sur les applications en imagerie médicale de la RMN.

\section{ii. Le bruit}

Le bruit correspond à la présence de signaux parasites qui dégradent la qualité de l'image obtenue. II provient de différents facteurs internes ou externes au dispositif : les imperfections du système de résonnance magnétique (inhomogénéités de B0 ou B1; bruit thermique de l'antenne ; non linéarité des amplificateurs) ; le traitement de l'image ; le bruit physiologique (mouvements respiratoires, cardiaques...) ; le bruit de radiofréquence provenant de l'environnement. On définit le rapport signal sur bruit (RSB), exprimé en décibels $(\mathrm{dB})$, comme l'indicateur de la qualité de la transmission de l'information. II correspond au rapport des puissances entre le signal d'amplitude maximale pour la laquelle la distorsion reste inférieure à une valeur limite et le bruit de fond qui correspond au signal présent à la sortie d'un dispositif en l'absence de signal d'entrée. L'amélioration du RSB repose sur l'optimisation des processus internes pour réduire les sources de bruit (nombre de composants, simplicité des circuits...), la limitation de la bande passante uniquement à la bande utile par filtrage, la modification du nombre d'excitation (Nex), la diminution de la température ou encore la réduction des perturbations extérieures par blindage électromagnétique. II s'agit pour certains d'éléments constructeurs ou propres à l'environnement de la machine IRM sortant du champ d'application de nos modèles expérimentaux. D'autres paramètres, comme en particulier la confection d'antennes d'acquisition parallèle (présenté dans résultats), 
ont fait l'objets d'un investissement particulier des équipes de recherche pour permettre d'améliorer le RSB et d'accélérer l'acquisition.

\section{iii. La résolution}

La résolution spatiale permet de déterminer la dimension du plus petit détail observable par l'œil. Elle dépend directement de la taille d'un voxel. Le voxel dépend lui-même de 3 critères : l'épaisseur de la coupe IRM (il grandit avec lui), la taille du champ de vision (CV) (à matrice égale, il augmente au carré), la taille de la matrice (à CV équivalent, il diminue au carré). Ainsi, plus petit est le voxel, meilleure est la résolution spatiale. Toutefois, cela s'accompagne nécessairement d'une diminution du RSB. II s'agit donc de trouver le compromis idéal pour permettre de visualiser les détails nécessaires au diagnostic tissulaire.

Le signal étant proportionnel au volume du voxel, le RSB diminue donc rapidement. La compensation de cette perte par une accumulation de signaux augmente considérablement le temps d'acquisition de l'image. Pour répondre à cette contrainte, il est donc préférable d'améliorer la sensibilité d'acquisition. Ceci peut être réalisé soit en augmentant l'intensité du champ magnétique, soit en utilisant des antennes plus sensibles en raison de leur petite taille (ex. : réseaux d'antennes) ou du matériau dont elles sont faites (supraconducteur). L'ensemble des paramètres influençant la résolution spatiale est présentée dans le Tableau 2.

Ainsi, l'augmentation de l'intensité du champ magnétique entraîne un gain de RSB qui diminue la taille du voxel et augmente ainsi la résolution spatiale. En pratique clinique pour l'imagerie de la prostate, cela conduit simultanément à réduire les effets de 
moyennage dus à la graisse péri-prostatique, aux hémorragies post-biopsiques, aux vésicules séminales, aux nodules d'hyperplasie bénigne et au tissu péri-urétral. Les résultats initiaux de l'imagerie spectroscopique endorectale $3 \mathrm{~T}$ 1H-MR chez les patients atteints de cancer de la prostate ont montré à ce sujet des avantages potentiels : augmentation de la résolution spatiale, temporelle et spectrale à un champ plus élevé pouvant améliorer la précision du cancer de la prostate. Mais l'augmentation de l'intensité des champs magnétiques s'accompagnait dans cette étude de modifications de paramètres physiques potentiellement sources d'artéfacts [41] qu'il est nécessaire d'appréhender pour définir des réglages compensateurs.

Tableau 2 - Paramètres influençant la résolution spatiale

\begin{tabular}{|c|c|c|}
\hline Paramètre & Augmenté & Diminué \\
\hline TR & $\begin{array}{c}\text { RSB }+ \\
\text { Plus de coupes }\end{array}$ & $\begin{array}{c}\text { Durée d'acquisition - } \\
\text { Contraste T1 + }\end{array}$ \\
\hline TE & Contraste T2+ & $\begin{array}{c}\text { RSB + } \\
\text { Plus de coupes }\end{array}$ \\
\hline B0 & RSB + & Artefacts - \\
\hline Nex & $\mathrm{RSB}+$ & Durée d'acquisition - \\
\hline Épaisseur de coupe & $\begin{array}{c}\mathrm{RSB}+ \\
\text { Large couverture }\end{array}$ & $\begin{array}{l}\text { Résolution spatiale + } \\
\text { Volume partielle - }\end{array}$ \\
\hline Espace inter-coupe & $\begin{array}{l}\text { Large couverture } \\
\text { Bruit - }\end{array}$ & Détection + \\
\hline Matrice & Résolution spatiale + & $\begin{array}{c}\text { RSB+ } \\
\text { Durée d'acquisition - }\end{array}$ \\
\hline Champs visuel & $\begin{array}{c}\text { Large couverture } \\
\text { RSB + } \\
\text { Artefact - }\end{array}$ & Résolution spatiale + \\
\hline
\end{tabular}

Abréviations : TR : temps de répétition ; TE : temps d'écho ; Nex : nombre d'excitations 


\section{Enjeux \& Objectifs des travaux}

Enjeu général : dépasser les limites actuelles de l'IRM clinique en termes de résolution spatiale pour améliorer son rendement diagnostique grâce à l'apport d'une imagerie haute résolution par IRM.

- Premier objectif : travaux expérimentaux 1

Décrire un modèle expérimental sur des échantillons de prostate humaines pour définir les paramètres d'optimisation de séquence IRM nécessaires à l'obtention d'une résolution spatiale proche de celle obtenue par un grossissement histologique satisfaisant pour faire un diagnostic anatomo-pathologique du cancer de la prostate.

- Deuxième objectif : travaux expérimentaux 2

Reproduire par une nouvelle série d'expériences, une imagerie IRM haute résolution selon les paramètres de séquences IRM optimisées, précédemment définis, sur une série d'échantillons de prostatectomie radicale entière pour valider les résultats préliminaires et réaliser un atlas sémiologique d'imagerie IRM haute résolution 7T humaine de prostate en comparant les images aux coupes histologiques correspondantes finales. 


\section{Modèle et Travaux expérimentaux 1}

II s'agissait d'une étude clinique expérimentale prospective, non randomisée monocentrique d'imagerie par IRM haute résolution d'échantillons de prostates humaines ex vivo après prostatectomie radicale de patients consécutifs consentants opérés pour cancer ou de donneurs d'organes décédés. Ce travail dans sa plus large partie a déjà été publié.

\section{a. Objectif Principal}

L'objectif principal était de déterminer les paramètres du protocole d'imagerie IRM haute résolutions pour permettre la caractérisation à des échelles spatiales de longueur histologique à des fins diagnostiques dans des conditions de laboratoires en utilisant les coupes anatomo-pathologiques correspondantes comme standard de référence.

\section{b. Cohorte d'étude}

Les échantillons étudiés correspondaient à 15 prostates différentes qui ont pu être collectées au cours de l'étude. Elles provenaient de $n=3$ donneurs d'organes décédés en état de mort encéphalique ayant bénéficiés de prélèvement multi-organe incluant la prostate, et $n=12$ patients opérés de prostatectomie radicale laparoscopique robotassistée programmée pour la prise en charge de leur cancer localisé de prostate. Les patients atteints de cancer de prostate cliniquement localisé ou localement avancé (T3a maximum) avaient au préalable suivi des investigations préopératoires complètes incluant : dosage de PSA, IRM multiparamétrique de prostate, série de biopsies avec au moins une carotte positive avec analyse du score Gleason, bilan d'extension 
comprenant, en fonction des recommandations en vigueur, un scanner thoracoabdomino-pelvien injecté et une scintigraphie osseuse $\mathrm{Tc}^{99 \mathrm{~m}}$ en l'absence de contreindication.

\section{c. Cadre réglementaire de déclaration d'étude}

Cette étude avait été validée par le comité d'éthique institutionnel (institutional review board - IRB) de The New York Presbyterian Hospital-Weill Medical College of Cornell University (WCMC), enregistrée sous le numéro de protocole \#0701008945, sous le titre de dépôt de projet « High Resolution MRI Studies of Prostate Tissue Specimens ». Elle a été ouverte pour une durée de 1 an de 2015 à 2016. Les financements provenaient du département d'Urologie. Les travaux étaient menés sous la responsabilité de l'investigateur principal, Ashutosh Tewari, MD, Associate Professor of Urology. L'ensemble des patients de prostatectomie radicale avait signé un consentement après avoir reçu une information complète et adaptée sur le protocole. Les risques encourus présentés dans le cadre de cet essai étaient principalement la perte d'information sur le cancer de prostate, sachant les analyses sur pièces fraîches pouvant détériorer le matériel prélevé et sachant le risque de perte de l'échantillon dans les transferts entre laboratoires. Les ayant-droits des patients donneurs en état de mort encéphalique avaient signés également une permission propre à ce circuit ; aucun risque n'avait été identifié pour cette source d'échantillon.

L'ensemble des dossiers de ce protocole était conservé dans le bureau d'étude fermé du coordonnateur de recherche et / ou de l'investigateur principal pendant toute période de ce protocole. L'accès aux dossiers d'étude était réglementé et limité à ceux qui travaillaient directement sur le projet ou à ceux qui en avait besoin (exemple : 
Vérificateurs de la conformité, etc.). Le comité d'examen institutionnel et les comités de surveillance fédéraux pouvaient disposer d'un accès à ces données. Le transfert des données entre les départements (radiologie à l'urologie et vice \& versa) s'est fait par livraison manuelle par le personnel d'étude. L'identité des sujets n'a été divulguée ni publiée de quelque manière que ce soit.

\section{d. Consentements patients \& risques}

Les consentements éclairés écrits et signés étaient obtenus par le chirurgien urologue au moment de la programmation du geste chirurgical de prostatectomie radicale indiqué pour les patients atteints de cancer de prostate. Dans le cas des donneurs, c'était l'équipe de coordination des prélèvements d'organes qui présentaient le dispositif à la famille ou aux ayant-droits lors d'anticipation du diagnostic de la mort cérébral. Les sujets ou leurs ayants-droits étaient alors informés qu'il s'agissait d'une étude de recherche volontaire, s'ils choisissaient de participer. Chaque sujet inscrit à l'étude ou ses ayant-droit était avisé qu'il était possible que l'échantillon prélevé pendant la chirurgie puisse être perdu et / ou endommagé pendant le transport entre la chirurgie, la radiologie et le service d'anatomo-pathologie. Dans le cas peu probable où cela aurait pu se produire, cela aurait empêcher tout ou partie de l'analyse diagnostique par le département d'anatomopathologie pour les patients opérés de cancer de prostate avec un risque de pertes d'informations médicales pouvant altérer leur projet personnalisé de soins.

e. Préparation, manipulations \& suivi des échantillons prostatiques pour imagerie 
Imagerie des glandes entières ex vivo. Des glandes de prostatectomie entières excisées fraîches provenaient de donneurs d'organes $(N=3)$ ou de sujets ayant subi une prostatectomie radicale robotique $(N=6)$. Après résection par le chirurgienurologue investigateur, l'échantillon une fois sélectionné était immédiatement conditionné au bloc opératoire (Greenberg 3) dans une compresse imbibée de sérum physiologique, scellées dans un sac en plastique, pour être adressée à l'état frais, par le coordonnateur de recherche, sous $30 \mathrm{~min}$ au Centre d'imagerie biomédicale Citigroup (Citigroup Biomedical Imaging Center - CBIC), situé au 516 East 72nd Street, pour l'IRM. Dans ce cas d'imagerie de glande complète, la prostate entière était placée dans une bobine d'imagerie radiofréquence (qui agissait comme une antenne) et numérisée, telle quelle. Si une solution saline inadéquate était présente autour de l'échantillon, une solution saline stérile supplémentaire pouvait être ajoutée. À aucun moment, il n'y avait de coupe ou de résection de tissu prostatique.

Imagerie des coupes de tissus ex vivo. Les échantillons de prostatectomie radicale robotique $(\mathrm{N}=6)$ étaient d'abord soumise à une analyse anatomo-pathologique chirurgicale extemporanée pour un examen macroscopique. Après encrage de l'échantillon selon un protocole standard, la glande prostatique fraîche, c'est-à-dire avant la fixation au formol, était coupée de l'apex à la base en tranches de $5 \mathrm{~mm}$ d'épaisseur à la lame froide, selon un plan perpendiculaire à la face postérieure de la prostate sur toute sa hauteur (sans respect du protocole de Stanford). Ces tranches étaient inspectées visuellement à la recherche de la présence de foyers tumoral(aux). L'évaluation macroscopique de la localisation tumorale de ces coupes était réalisée avec l'assistance cartographique biopsique préopératoires couplée à celle de la cartographie PI-RADS des IRM préopératoires. La section de tissu frais présentant la 
plus forte probabilité de tumeur était sélectionnée pour imagerie. Selon la taille de la section, la section était divisée en deux ou en quatre pour s'adapter à l'intérieur d'une cassette d'anatomo-pathologie standard mesurant environ $30 \times 27 \times 5 \mathrm{~mm}^{3}$. Cette cassette était ensuite immergée dans une solution contenant un mélange de solution sérum salé physiologique à $0,9 \%$ et de Gadolinium d'acide diéthylènetriamine pentaacétique trisodique (Gd-DTPA) à $1 \%$, et délivrée par transporteur au centre d'imagerie de recherche.

Pour les glandes prostatiques entières et les sections de la procédure prostatectomie radicale robotique, toute l'imagerie était réalisée dans les 3 heures suivant l'excision en accord avec le temps d'imagerie approuvé par l'IRB. Pour les échantillons prélevés sur des donneurs d'organes, aucune limite temporelle n'avait été fixée pour l'imagerie.

A noter pour les pièces issues de prostatectomies radicales robotiques, afin de minimiser le temps de déplacement entre le département de chirurgie et celui d'anatomo-pathologie, le chirurgien-urologue devait prendre toutes les dispositions nécessaires pour que l'échantillon soit adressé le plus rapidement possible dès résection et conditionnement de la pièce pour être soumis rapidement au département de radiologie. Pour ce faire, lors des prostatectomies radicales de patients atteints de cancer de prostate, on avait recours à l'utilisation systématique d'un anneau rétracteur de plaies chirurgicales de type Alexis de taille S (Applied Medical, CA, USA). Introduit au niveau de l'incision péri-ombilical, il était recouvert d'un obturateur hermétique permettant la mise en place du trocart de $8 \mathrm{~mm}$ robot pour fixer la caméra. Dès ablation de la prostate, à la section de l'urètre prostatique, la pièce était placée dans un sac stérile, de type endobag de $600 \mathrm{~mL}$, et extraite au travers de l'Alexis pour être confiée 
immédiatement au coordonnateur de recherche. Ce faisant, on permettait de réduire au minimum les délais pour permettre l'analyse d'imagerie, sans attendre la fin de l'intervention, tout en diminuant au maximum les risques de léser les tissus frais.

Les échantillons de prostate recevaient tous un numéro d'identification anonymisé spécifique à l'étude une fois qu'ils étaient retirés du patient. Des feuilles de notation étaient préparés par échantillon pour suivre tous les spécimens au cours des transferts entre départements (chirurgie, radiologie et anatomo-pathologie). En quittant le département de chirurgie, la feuille de journal était horodatée par machine dans la salle opératoire du bloc par mesure d'identito-vigilance et de traçabilité. Tous les efforts étaient faits pour assurer un transfert efficace entre départements et pour éviter la perte ou l'erreur d'identification d'échantillon.

\section{f. Critères de jugement \& mesure des objectifs}

La résolution spatiale se définit par la capacité de discerner spatialement deux structures proches. Elle correspondait à la mesure de la finesse des détails d'une image pour une dimension donnée. Elle était appréciée par le champ de vue (FOV), exprimé en mm, et la matrice d'acquisition, exprimée en pixels. Pour augmenter la résolution spatiale, on diminuait la bande passante d'acquisition, augmentant de fait le RSB mais également le décalage chimique. On pouvait aussi augmenter l'intensité du gradient du champs magnétique. L'intensité du champ magnétique étant proportionnel à la fréquence de résonnance, les voxels situés à différentes distances le long du gradient avaient des fréquences de résonance différentes. Les voxels se définissent comme un cube formé par un pixel et l'épaisseur (= profondeur) de la coupe. 
Le temps d'acquisition était colligé et variait selon les analyses. Il dépendait du nombre d'acquisitions pour moyenner (NSA), du temps de répétition (TR), du nombre de pas d'encodage de phase $\left(\mathrm{N}_{\mathrm{PE}}\right)$, du nombre de coupes (la dépendance est fonction des paramètres de la séquence) ; au total, le temps d'acquisition $\propto$ NSA.TR.NPE.

La bande passante correspondait à la largeur de la gamme de fréquences autour de la fréquence le Larmor. Elle était exprimée en $\mathrm{KHz}$, en $\mathrm{Hz}$ par pixel, ou en nombre de pixel entre l'eau et la graisse. Le RSB dépendait de la taille du pixel, de la séquence et du tissu. Signal $\propto \Delta \mathrm{x} \Delta \mathrm{y} \Delta \mathrm{zF}_{\text {sep-tissu. }}$

Le contraste s'exprimait par la capacité de discerner un tissu distinct d'un autre. II dépendait des paramètres de la séquence (TE, TR, TI, FL). L'affichage finale correspondait au réglage du contraste à l'écran par le choix du centre et de la largeur de la largeur de la fenêtre.

Pour permettre l'étude d'optimisation d'imagerie IRM, l'ensemble des paramètres suivant étaient modifiés et conservés: champs magnétique statique, antenne, contraste, FOV et résolution spatiale, temps d'acquisition, NSA, largeur de bande.

\section{g. Protocoles d'imagerie}

IRM 3T haute résolution. Les images de la glande entière ont été acquises en utilisant un système d'imagerie par résonance magnétique SIGNA EXCITE de General Electric (Fairfield CT, USA). Ce système a été choisi car il était également utilisé pour l'imagerie in vivo et offrait ainsi le même choix de paramètres d'imagerie pour les échantillons excisés. Un résonateur d'émission / réception solénoïde à enroulement parallèle a été utilisé pour la transmission et la réception. Les détails de la conception du résonateur ont été décrits précédemment [42]. Une séquence d'écho de spin a été appliquée en utilisant des paramètres d'impulsion conçus pour produire un contraste 
d'image presque identique à celui obtenu pour l'imagerie clinique de la prostate (protocoles A et B). Des projections d'intensité maximale ont été appliquées aux tranches acquises à partir de la glande entière.

IRM 7T haute résolution. Les images ont été acquises sur un système d'imagerie par résonance magnétique BioSpec® (Bruker Biospin, Billerica, MA, USA) conçue pour l'expérimentation préclinique composé d'un tunnel de $30 \mathrm{~cm}$ de diamètre, équipé d'une antenne de gradient B-GA $12 \mathrm{~S}$ évalué à $450 \mathrm{mT} / \mathrm{min}$. Pour l'imagerie de la glande entière, le résonateur était similaire à celui de l'IRM 3T. Pour l'imagerie des sections, un prototype spécifique avait été construit pour permettre l'acquisition d'images selon le plan exact des coupes histologiques finales. II consistait en une plate-forme de bois en pin, isolé du trou de l'aimant par des cylindres de mousse en polyéthylène placés à chaque extrémité. Sur sa surface supérieure reposait verticalement un récipient rectangulaire transparent en acrylique, type boîte de Tic-tac $₫$, ouvert vers le haut de façon à contenir une cassette d'histologie aux dimensions extérieures de $41 \times 29 \times 7$ $\mathrm{mm}^{3}$ et le liquide d'immersion. Un résonateur solénoïdal parallèle s'enroulait sur 3 tours autour de la boîte, fixé au récipient par l'intermédiaire d'un adhésif appliqué sur la feuille de cuivre comprenant les boucles. Un diagramme de l'appareil est représenté sur la Figure 5. Au total, 5 protocoles d'IRM 7T (protocole C à G) ont été appliqués pour l'imagerie de sections prostate ou de glande entière ex vivo. De multiples techniques d'acquisition d'écho de spin et d'écho de gradient ont été testés.

Trois acquisitions séparées d'écho de gradient à faible angle de tir (FLASH) ont été utilisées. Le premier (protocole E) était une acquisition bidimensionnelle et a été utilisé uniquement pour évaluer rapidement la perméabilité de la section de la glande à l'agent de contraste avec un temps de balayage de 7 minutes, 47 secondes et une 
résolution spatiale de $107 \times 107 \times 500 \mu \mathrm{m}^{3}$. La seconde séquence d'écho de gradient FLASH tridimensionnelle (protocole F) a été utilisée pour l'imagerie de spécimens pendant une nuit complète avec la plus haute résolution sur une durée de 8 heures, 36 minutes à $60 \times 60 \times 60 \mu \mathrm{m} 3$. La troisième acquisition FLASH tridimensionnelle (protocoles G) a été sélectionnée pour l'imagerie des échantillons de prostatectomie radicale robot avant évaluation histologique pour les soins courant avec des paramètres identiques au protocole $\mathrm{F}$ à l'exception de la taille de la matrice de $1024 \mathrm{X}$ $1024 \times 128$, à une résolution spatiale de $60 \times 60 \times 90 \mu \mathrm{m} 3$. Le protocole $\mathrm{G}$ avait NEX $=3$ et était une acquisition de 2 heures et 51 minutes. Les deuxième et troisième séquences FLASH ont permis une couverture complète de l'échantillon de $5 \mathrm{~mm}$ d'épaisseur, ce qui a facilité la comparaison ultérieure avec l'histologie. L'ensemble des protocoles expérimentaux est synthétisé dans le Tableau 3. 

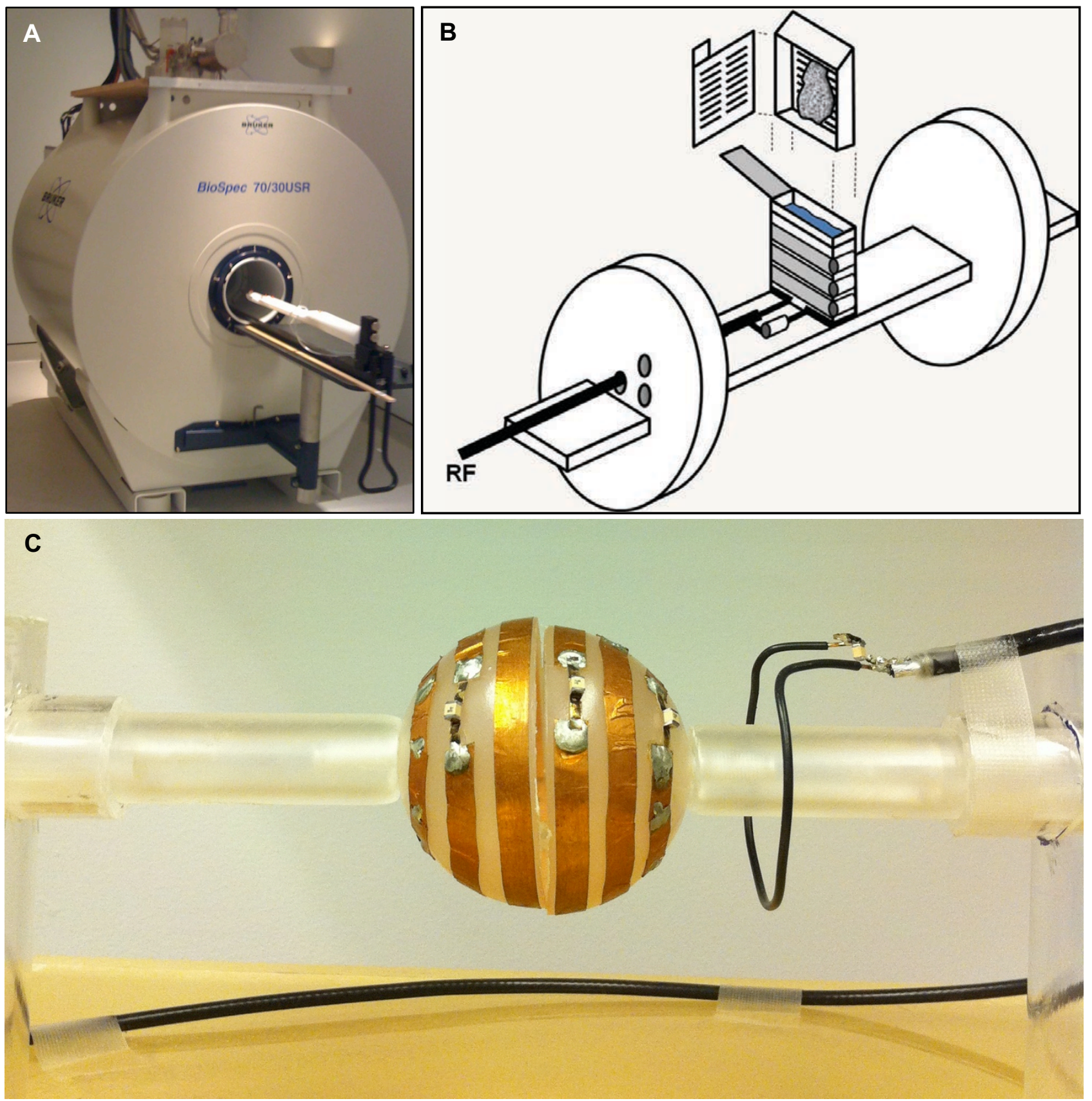

Figure 5 - IRM expérimentale \& antennes prototypes

(A) IRM 7 Tesla Bruker Biospec $®$ (Bruker Biospin, Billerica, MA, États-Unis) Système d'imagerie par résonance magnétique de $30 \mathrm{~cm}$ de diamètre équipé de bobines de gradient B-GA $12 \mathrm{~S}$ évaluées à $450 \mathrm{mT} / \mathrm{m}$. (B) L'appareil utilisé pour l'imagerie des sections de la prostate à 7 Tesla. Le résonateur solénoïdal à enroulement parallèle à trois boucles utilisait du cuivre avec une largeur de $12,7 \mathrm{~mm}$ et une épaisseur de $66 \mu \mathrm{m}$ (Société $3 \mathrm{M}$ ). Les condensateurs variables avaient des valeurs de 3-40 pF. D'autres caractéristiques de l'appareil comprennent des supports en mousse de polyéthylène pour un positionnement précis de l'échantillon à l'isocentre de l'aimant, une plate-forme en bois de pin pour le résonateur et la cassette (avec couvercle enlevé), un condensateur variable sur chacune des trois boucles de résonateur, une boucle à couplage inductif avec condensateur variable pour l'adaptation d'impédance à la ligne de transmission 50 ohms, un réservoir pour le mélange salin et Gd-DTPA dans lequel la cassette et l'échantillon étaient immergés, et enfin une cassette identique à celle utilisée pour l'échantillon préparation en pathologie. (C) IRM 7 Tesla MRI antenne sphérique construit sur mesure pour les spécimens de prostatectomie radicale robot ex vivo en utilisant un résonateur d'émission / réception solénoïde à enroulement parallèle. Un résonateur similaire a été utilisé pour l'imagerie à 3 Tesla. 
Tableau 3 - Protocoles de paramètres d'acquisition d'imagerie de haute résolution 3 Tesla et 7 Tesla IRM

\begin{tabular}{|c|c|c|c|c|c|c|c|c|c|c|c|c|c|c|}
\hline $\begin{array}{l}\text { \# Protocole } \\
\text { Imagerie }\end{array}$ & Spécimen & $\begin{array}{l}\text { CM } \\
\text { (Tesla) }\end{array}$ & Pulse Sequence & $A C Q$ & $\begin{array}{l}\text { TR } \\
\text { (ms) }\end{array}$ & $\begin{array}{l}\text { TE } \\
\text { (ms) }\end{array}$ & $\begin{array}{l}\text { CV } \\
\text { (cm) }\end{array}$ & $\begin{array}{l}\text { Matrice } \\
\text { Acquisition } \\
\text { (cm) }\end{array}$ & $\begin{array}{l}\text { Flip } \\
\text { Angle }\end{array}$ & $\begin{array}{l}\text { Épaisseur } \\
\text { Coupe } \\
\text { (mm) }\end{array}$ & $\begin{array}{l}\text { Echo } \\
\text { Train }\end{array}$ & NEX & $\begin{array}{l}\text { Temps } \\
\text { (h:min:sec) }\end{array}$ & $\begin{array}{l}\text { Résolution } \\
\left(\mu \mathrm{m}^{3}\right)\end{array}$ \\
\hline A & Glande Entière & 3 & SE & $2 \mathrm{D}$ & 5066 & 105 & 6,0 & 320,192 & 90 & 1.0 & 20 & 4 & $0: 13: 52$ & $188 \times 312 \times 1000$ \\
\hline B & Glande Entière & 3 & SE & $2 \mathrm{D}$ & 6067 & 171 & 6,0 & 320,192 & 90 & 1.0 & 22 & 3 & 0:08:30 & $188 \times 312 \times 1000$ \\
\hline C & Glande Entière & 7 & SE (RARE) & $2 \mathrm{D}$ & 2000 & 84,9 & 5,5 & 512,512 & 90 & 0.75 & 24 & 16 & 0:11:00 & $107 \times 107 \times 750$ \\
\hline D & Section Prostate & 7 & SE (RARE) & $2 \mathrm{D}$ & 2000 & 75,8 & 5,5 & 1024,1024 & 90 & 0.50 & 12 & 14 & $0: 39: 40$ & $54 \times 54 \times 500$ \\
\hline E & Section Prostate & 7 & GRE (FLASH) & $2 \mathrm{D}$ & 101,4 & 5,5 & 5,5 & 512,512 & 40 & 0.50 & NA & 12 & $0: 07: 47$ & $107 \times 107 \times 500$ \\
\hline $\mathbf{F}$ & Section Prostate & 7 & GRE (FLASH) & $3 \mathrm{D}$ & 26,3 & 13,1 & 6,0 & $\begin{array}{l}1024, \quad 1024, \\
192\end{array}$ & 15 & 0.06 & NA & 6 & 8:36:00 & $60 \times 60 \times 60$ \\
\hline G & Section Prostate & 7 & GRE (FLASH) & $3 \mathrm{D}$ & 26,3 & 13,1 & 6,0 & $\begin{array}{l}1024, \quad 1024 \\
128\end{array}$ & 15 & 0.06 & NA & 3 & 2:51:00 & $60 \times 60 \times 90$ \\
\hline
\end{tabular}

Abréviations : AB : angle de bascule ; ACQ : acquisition ; CM : champ magnétique ; CV : champ de vision ; EGR : écho de gradient ; FLASH : fast low angle shot ; LTE : longueur du train d'écho ; ES : écho de spin ; MA : matrice d'acquisition ; NEX : nombre d'excitations ; NA : non applicable ; SI : séquence d'impulsion ; T : Tesla ; TE : temps d'écho ; Tps : temps ; TR : temps de répétition 


\section{h. Analyse anatomo-pathologique et étude de corrélation}

Après réalisation de l'imagerie, l'ensemble des échantillons utilisés étaient adressés sous scellé dans un bain de formol tamponné à $10 \%$ au département d'anatomopathologie pour permettre l'analyse histologique finale des différentes pièces. L'étude tissulaire suivait les recommandations techniques du protocole standard de Stanford pour les prostates entières et un protocole modifié pour les sections de prostates qui étaient orientées selon l'ancrage initial réalisé avant coupe.

En cas de tumeur identifiée, la lésion était entourée sur la lame manuellement au feutre et le score de Gleason était réalisé après coloration H\&E par l'uroanatomopathologiste. Secondairement, les lames comportant les coupes de cancer de prostate diagnostiqué étaient numérisées avec un scanner à plat pour permettre le travail de comparaison avec les images IRM correspondantes par inspection visuelle. Aucun système de recalage validé n'était utilisé. Le matching était visuel entre les coupes IRM et les coupes anatomopathologiques. Pour les glandes complètes, leurs analyses histologiques étaient réalisées comme indiquées ci-dessous, mais aucune étude de comparaison avec les images IRM n'était attendue compte-tenu de l'absence de repère tridimensionnel nécessaire pour permettre de comparer selon le même plan. 


\section{i. Résultats}

Le protocole d'imagerie A à 3 Tesla nécessitait un temps de balayage de 14 minutes pour recouvrir toute la glande en 85 tranches, et donnait une résolution spatiale de $188 \times 312 \times 1000 \mu \mathrm{m}^{3}$ comme présentée sur la Figure 6B avec une section de la glande obtenue après prostatectomie radicale. Ceci est comparé à l'imagerie préopératoire à 1,5 T (Figure 6A, à une résolution clinique de $547 \times 625 \times 3000 \mu \mathrm{m}^{3}$ ). Alors que les détails sont améliorés sur la figure $6 \mathrm{~B}$, des zones supplémentaires de signal hypo-intense apparaissent dans la zone périphérique, ce qui peut potentiellement fausser le diagnostic. Dans la préparation histologique de la Figure 6C, la zone hypo-intense en bas à gauche contient du cancer de prostate avec un score de Gleason $7(4+3)$. La zone contenant le cancer est superposable à celle mise en évidence sur les résultats de la Figure 6A.

A une résolution spatiale plus élevée, le critère classique de diagnostic de la malignité sur les images pondérées en T2 devenait mois évident. Dans la Figure 6D, une section de la glande entière imagée à 7 Tesla est présentée en utilisant les paramètres d'acquisition RARE (protocole C). Cette image d'un échantillon différent est montrée à une résolution spatiale de $100 \times 107 \times 750 \mu \mathrm{m}^{3}$, obtenue avec un temps de balayage de 11 min. Beaucoup plus de détails sont évidents, car les zones de stroma entourant l'architecture ductale commencent à être élucidées. Les conduits individuels sont bien définis dans certains cas, et dans l'ensemble l'échelle de longueur fondamentale pertinente de la glande de la prostate commence à être approchée comme défini par la visualisation des structures morphologiques microscopiques importantes qui composent la glande. Cependant, comme pour le carcinome, le stroma est également défini par plusieurs zones d'hypointensité du signal. 

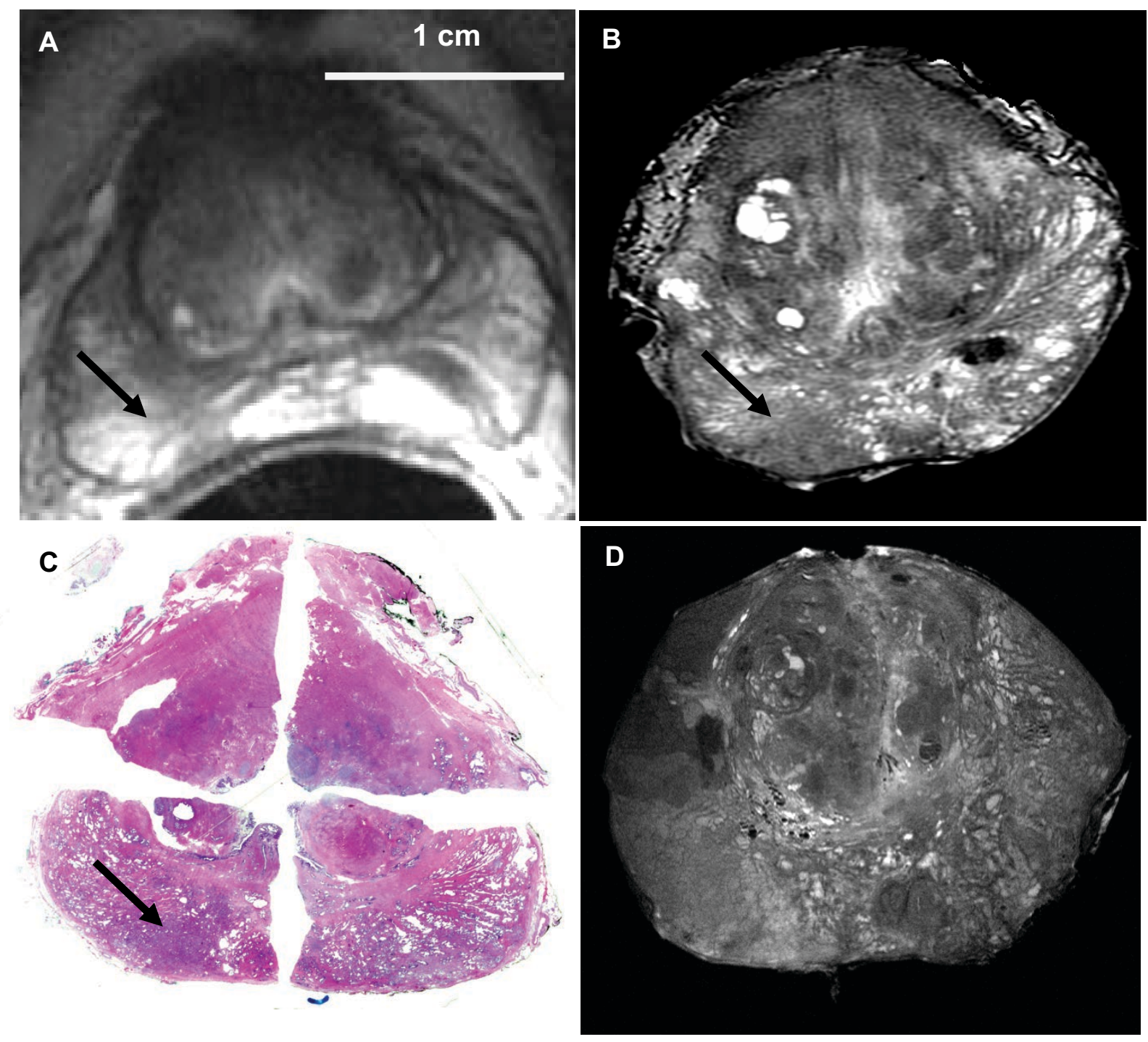

Figure 6 - Coupes transversales d'une prostate à différents niveaux de résolution spatiale IRM.

(A) IRM préopératoire séquence T2W à 1,5 Tesla à une résolution spatiale de $547 \times 625 \times 3000 \mu \mathrm{m} 3$ et un temps de balayage d'environ sept minutes. A cette résolution, seules l'image apparaît floues, aucune structure architecturale tissulaire n'est identifiable. On note un hypo-signal franc postéro-latéral médian droit (flèche), traditionnellement considéré comme suspect de malignité. $(\mathrm{B})$ Image IRM ex vivo à 3T de la même glande après prostatectomie radicale robot à une résolution spatiale de $188 \times 312 \times 1000 \mu \mathrm{m}^{3}$, obtenue en un temps de balayage d'environ 14 minutes (protocole A). L'image est plus nette. L'hyposignal de la zone postéro-latérale droite médiane est toujours présent. Alors que le niveau de détail architectural est amélioré, des zones supplémentaires en hyposignal sont présentes dans la partie jonctionnelle et périphérique contro-latérale. (C) Coupe histologique correspondante révélant la présence d'un cancer de prostate de score de Gleason $7(4+3)$ (flèche). (D) Imagerie 7 Tesla d'un échantillon différent à une résolution de $100 \times 107 \times 750 \mu \mathrm{m}^{3}$ dans un temps de balayage de 11 minutes (protocole $\mathrm{C}$ ) ; il révèle un haut niveau de détails de l'architecture tissulaire, et commence à élucider les zones de stroma entourant l'architecture ductale. 
La représentation globale de la glande a été complétée par une présentation de projection d'intensité maximale comme pour l'exemple présenté ci-après, obtenue en 8 minutes et 30 secondes à 3 Tesla (protocole $B$, Figure 7). Dans ce cas, la projection permet une bonne visualisation de multiples nodules arrondis lisses et variés dans la zone centrale indiquant une hypertrophie bénigne de la prostate (HBP). Dans la zone périphérique, le grossissement met en évidence la superposition de différentes couches concentriques correspondant à l'histologie des couches stromales prostatiques qui constitue la surface glandulaire.

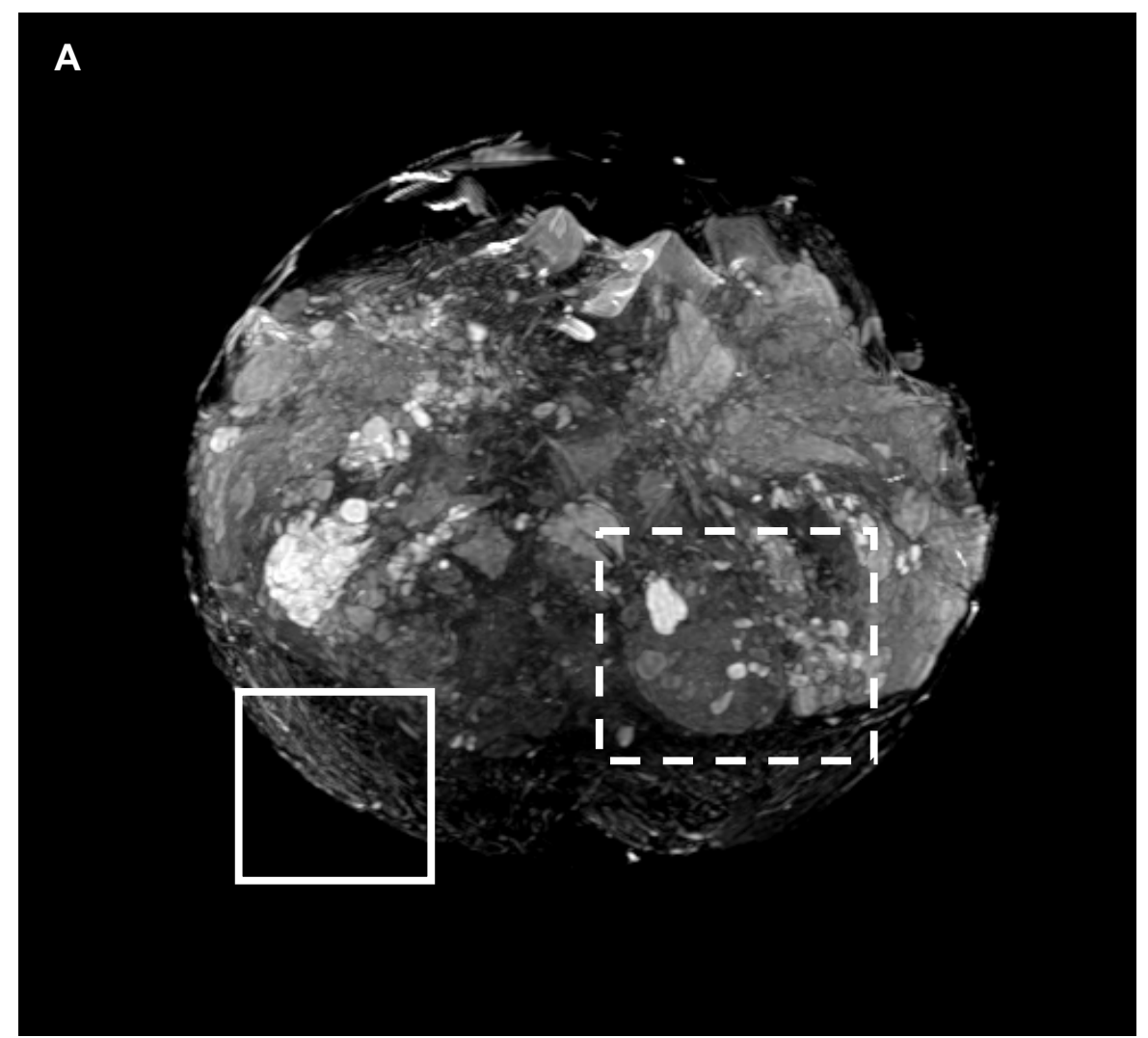

Figure 7 - Imagerie de projection d'intensité maximale d'une glande prostatique ex vivo entière

Elle a été obtenue en 8,5 minutes (protocole $\mathrm{B}$ ) à une résolution spatiale de $188 \times 312 \times 1000 \mu \mathrm{m}^{3}$ à 3 Tesla $(A)$. Les sous-structures glandulaires sont visibles à la fois dans les zones périphériques et centrales. L'agrandissement de la zone postéro-latérale moyenne périphérique droite $(\mathrm{B})$ permet de visualiser les couches concentriques superposables de la capsule. A cette définition, on ne perçoit pas plus d'information sur les structures qui la composent. L'agrandissement centré sur la zone centrale gauche $(C)$ révèle la présence d'une opacité nodulaire isointense composée d'éléments hyper-intenses et de corps en hypo-signal faisant évoquer le remaniement structurel au d'un nodule d'adénome de prostate.
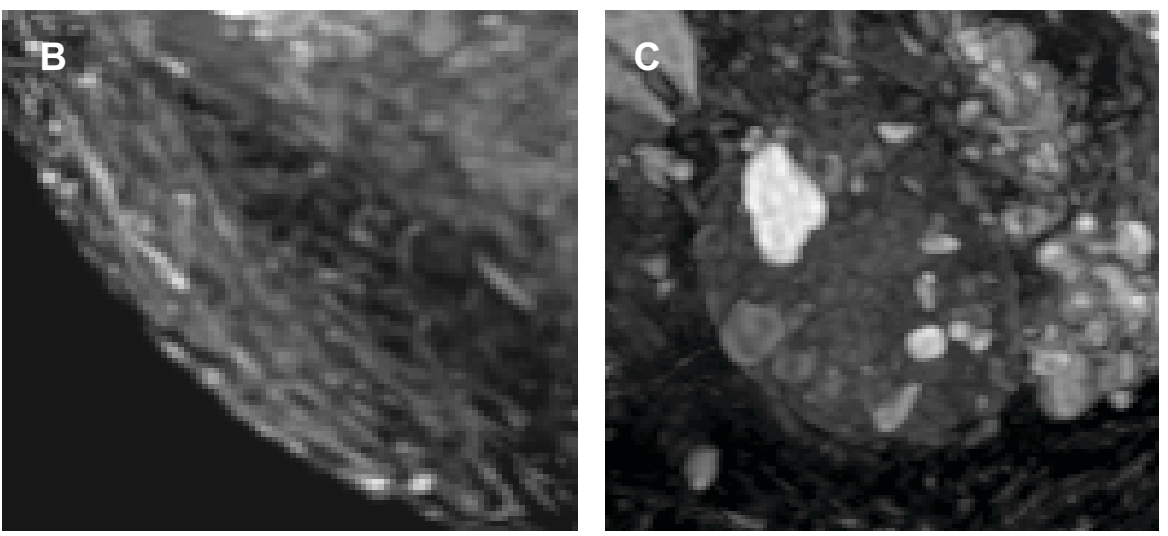
Une autre image d'écho de spin de 7 Tesla provenant d'un échantillon de glande entière est présentée sur la Figure 8. Elle met en évidence la vascularisation et la résolution de la paroi urétrale en utilisant une antenne de surface pour imager une petite zone. En utilisant le protocole $\mathrm{C}$ d'imagerie, les structures visibles s'approchent clairement de l'échelle microscopique comme le montre le grossissement à l'intérieur de la ligne pointillée blanche. La microanatomie du tissu intraprostatique est bien définie en IRM.
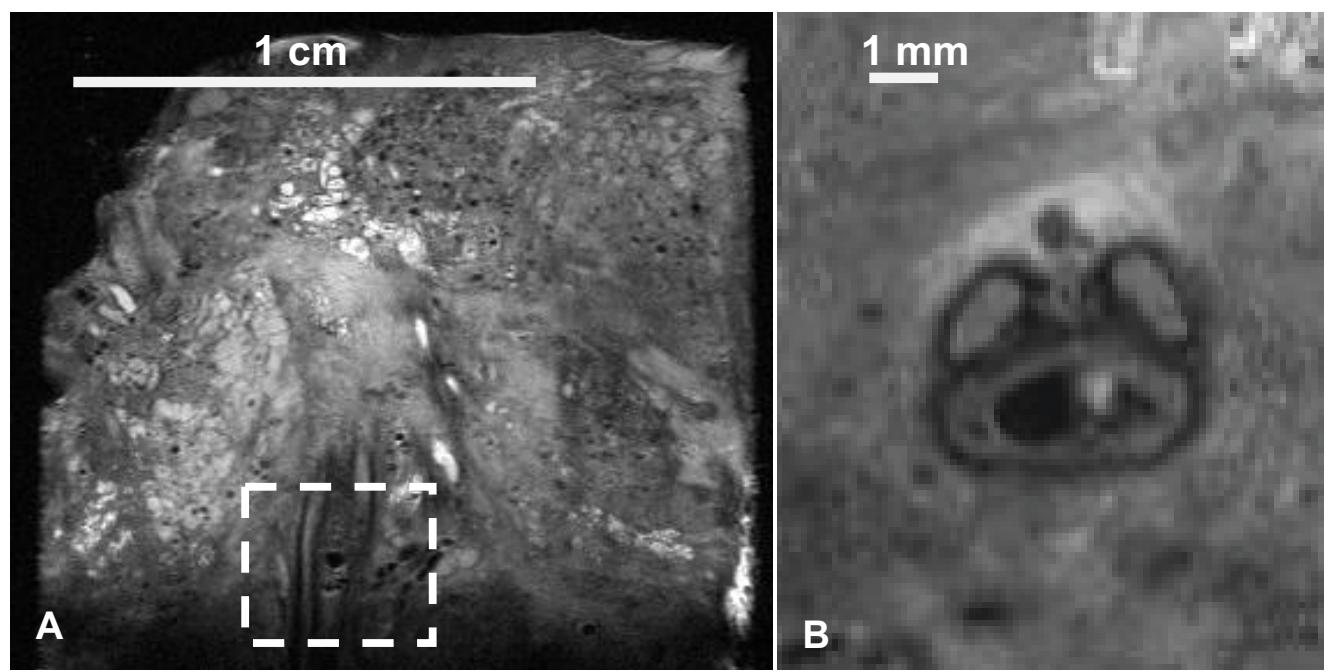

Figure 8 - Image d'écho de spin de 7 Tesla

II s'agit d'une glande entière à une résolution spatiale de $100 \times 107 \times 750 \mu \mathrm{m} 3$, obtenue avec un temps de balayage de $11 \mathrm{~min}$ (protocole $\mathrm{C}$ ). Cela met en évidence la vascularisation et la résolution de la paroi urétrale en utilisant une bobine de surface pour imager une petite zone (A). Les structures visibles s'approchent clairement de l'échelle microscopique comme le montre le grossissement à l'intérieur de la ligne pointillée blanche mettant en évidence la microanatomie du tissu intraprostatique $(B)$. 
Une image d'écho de spin d'un spécimen de prostate sectionné est présentée sur la Figure 9. II existe à la fois un enregistrement brut et spécifique des images IRM et H\&E, comme indiqué sur les Figures 9 B-D. Cette séquence a été obtenue après de balayage de 40 min avec une résolution spatiale de $54 \times 54 \times 500 \mu \mathrm{m}^{3}$, selon le protocole D. La technique d'écho de spin permettait de gommer une imperfection de type instrumental pour mieux mesurer la différence entre deux tissus ce qui en faisait un excellent critère pour la construction d'image. En particulier, ces images permettent de distinguer très précisément la microanatomie du tissu intraprostatique de façon bien définie. Les canaux individuels sont clairement visibles et délimitables sur l'imagerie IRM de façon tout à fait superposable à la coupe H\&E correspondante. Par grossissement, on perçoit l'architecture du tissu glandulaire, parfaitement représenté en IRM, avec des coupes d'élément de forme arrondie constitutif des glandes à sécrétion externe dites « en grappes » caractéristiques des tubulo-acini prostatiques décrites en H\&E, de taille approximative de 0,5 $\mu \mathrm{m}$. Dans leur lumière hyper-intense, on rapporte pour certains (flèches) la présence de petits corps irréguliers hypo-intense de $0,25 \mu \mathrm{m}$ superposables aux concrétions acineuses obstructives diagnostiquées sur l'histologie correspondante. Ces structures micro-anatomiques, dénommées sympexions prostatiques sont le résultat des sécrétions glandulaires naturelles, produites en couches concentriques, composées de glycoprotéines azotées pouvant se calcifier expliquant leur détection en hypo-signal IRM. 

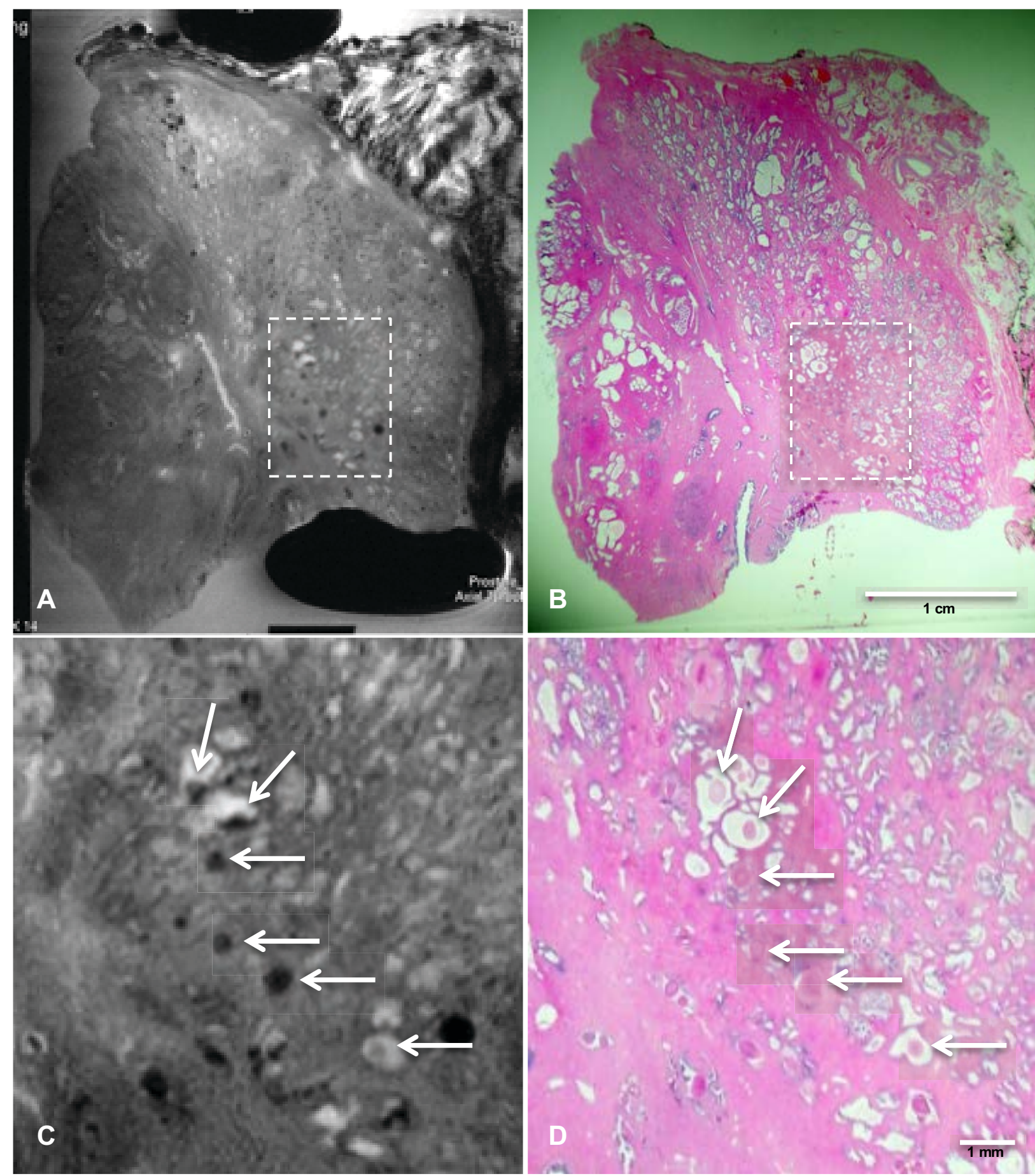

Figure 9 - Image d'écho de spin de 7 Tesla d'un échantillon de prostatectomie radicale.

(A) Image IRM d'écho de spin à une résolution spatiale de $54 \times 54 \times 500 \mu \mathrm{m}^{3}$, obtenue avec un temps de balayage d'approximativement 40 min (protocole D).

(B) Préparation histologique H\&E correspondante de l'échantillon de la figure 5A. La comparaison des deux images IRM et H\&E rend compte d'une exacte superposition de l'ensemble des micro-structures anatomiques qui composent et caractérise le tissu glandulaire sain prostatique légèrement cribriforme.

$(C, D)$ Vues agrandies des zones à l'intérieur des encadrés en pointillés des Figures $5 \mathrm{~A}, \mathrm{~B}$ qui rapportent la parfaite superposition structurelle sans perte d'information. L'IRM permet d'identifier des images corpusculaires arrondies de $500 \mu \mathrm{m}$ aux bords bien délimitées centrées par une lumière hyper-intense, correspondant aux acini décrits sur la coupe H\&E. Au sein de leur lumière (flèches), des corps arrondies d'hypo-intensité variable de $250 \mu \mathrm{m}$ parfois au contact des berges des acini sont identifiables et correspondent aux concrétions luminales en H\&E. 
Un spécimen provenant d'un donneur d'organes est présenté Figure 10. II est rapporté une imagerie de séquence en écho de gradient. Grâce à la réduction de l'angle de bascule propre à cette séquence, en général inférieur à $90^{\circ}$, on obtient une diminution de la quantité d'aimantation. Le retour à l'équilibre s'effectue donc plus rapidement du fait d'une aimantation résiduelle. En conséquence, les temps de répétition (TR) et temps d'écho (TE) employés ont pu être réduits de façon importante à 26,3 et 13,1 ms respectivement. Outre le gain de temps théorique, non rapporté dans notre étude, avec 8h environ de temps d'acquisition, cette séquence autorisait une imagerie de résolution de $60 \times 60 \times 60 \mu \mathrm{m}^{3}$ en $3 \mathrm{D}$ (protocole F). II était alors possible, comme précédemment en écho de spin, de discerner l'architecture tissulaire " en grappe » caractéristique du tissu glandulaire prostatique étroitement superposable à la coupes H\&E correspondante (Figure 10B). De plus, une succession stries hypointense en Figure $6 \mathrm{~A}$ évoquait la visualisation de la microvascularisation prostatique parenchymateuse péri-glandulaire confirmée par analyse histologique. La variation de projection d'intensité permise par cette séquence écho de gradient permettait d'apporter des variations de signal IRM complémentaires. Lorsqu'elle est traitée comme une image de projection d'intensité minimale à travers une pile 40 tranches obtenues à partir de l'écho de gradient tridimensionnel, le résultat révélait une image d'angiogramme IRM représentée sur la figure 10C, par une hyper-intensité périacineuse. Inversement, une projection d'intensité maximale à travers la même pile fournissait un ductogramme IRM de prostate en Figure 10D par un signal hyperintense suivant le cheminement acini et canaux spécifique spécifiques du tissu glandulaire prostatique sain. 

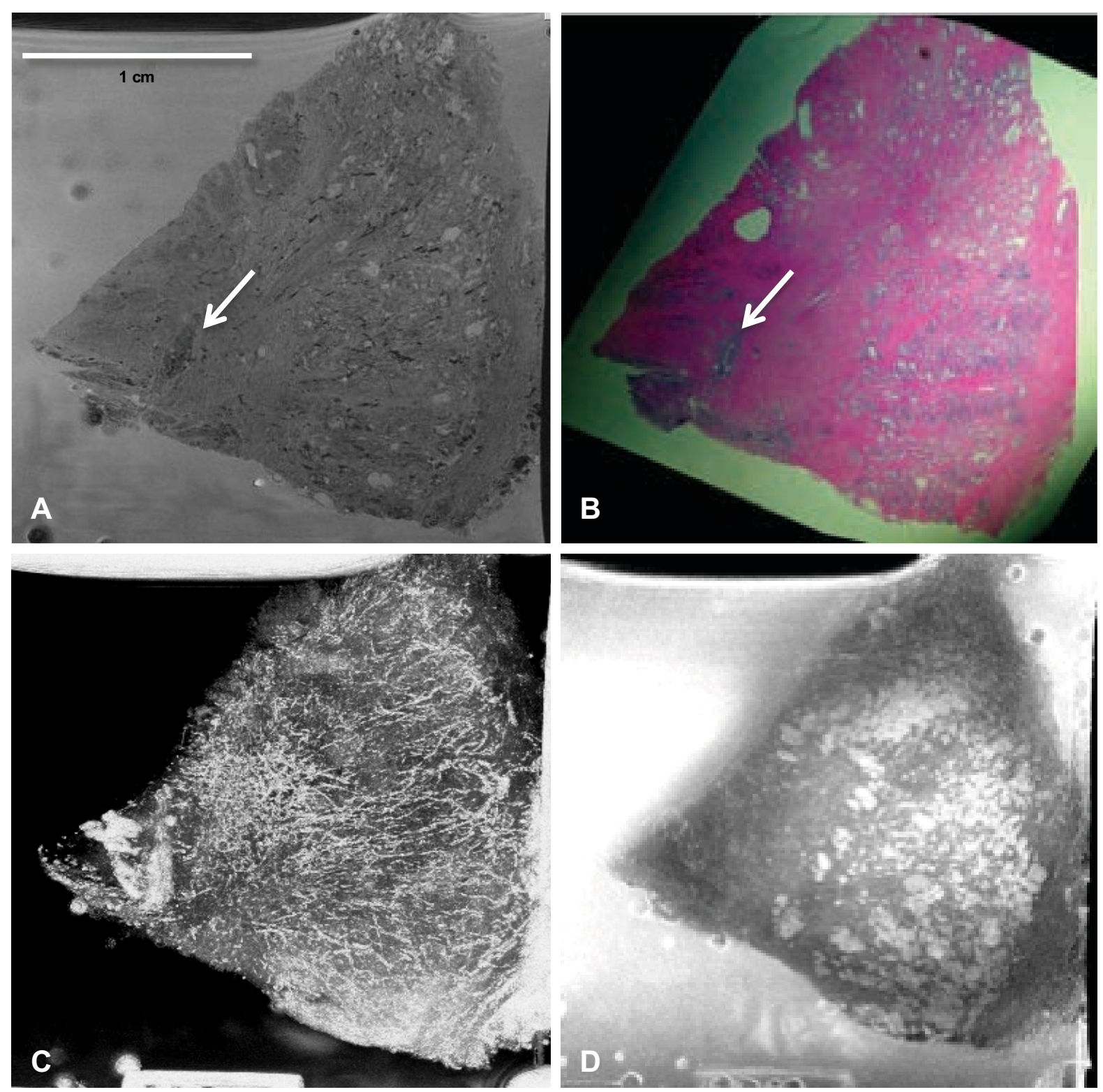

Figure 10 - Image en écho de gradient en IRM 7 Tesla d'un échantillon de prostate issu d'un donneur d'organe décédé.

L'image $(\mathrm{A})$ a été obtenue à partir d'une acquisition tridimensionnelle, à une résolution isotrope de $60 \times 60 \mathrm{X}$ $60 \mu \mathrm{m}^{3}$ en 8,5h approximativement (protocole F).

A cette résolution il est possible de discerner des agrégations spécifiques de cellules (flèches) comme confirmé en (B) la lame colorée H\&E correspondante.

De plus, les stries hypo-intense suggèrent une microvascularisation péri-acineuse prostatique qui, lorsqu'elle est traitée comme une image de projection d'intensité minimale sur l'ensemble de l'empilement d'environ 40 tranches obtenues à partir de l'écho de gradient tridimensionnel, produit l'angiographie présentée en (C). Inversement, une projection d'intensité maximale à travers la même pile fournit un ductogramme (D) hyperintense, représentation par imagerie de l'enchevêtrement des circonvolutions acineuses et canalaires du tissue glandulaire prostatique. 
Sur la Figure 11, une tentative a été faite avec le protocole $G$ de minimiser le temps d'acquisition à 7 Tesla tout en conservant les caractéristiques essentielles de la glande obtenus par les paramétrages précédents sur une image d'écho de gradient tridimensionnel. La figure $11 \mathrm{~A}$ a ainsi été obtenue avec une résolution légèrement plus importante par rapport au protocole $\mathrm{F}$, de $60 \times 60 \times 90 \mu \mathrm{m}^{3}$ en $1 \mathrm{~h}$ ou $3 \mathrm{~h}$ ( 2 tests identiques) après immersion de l'échantillon dans une solution de $\mathrm{NaCl}$ à $0,9 \%$ et de Gd-DTPA à 1,0\% pendant environ $1,5 \mathrm{~h}$ avant imagerie. La différence de résolution minime n'a pas modifié le niveau d'information obtenu sur IRM. Le contraste de l'image délimitait clairement l'architecture stromale et glandulaire avec des canaux intercalés (Figure 11A) superposables comme sur les résultats précédents en analyse histologique comparative (Figure 11B). La zone dans l'encart en pointillé présentait une variation de signal hypointense mal-délimitée avec perte des contours glandulaires habituellement bien souligné à cette résolution. Cette zone, en agrandissement sur la Figure 11D (flèche) correspondait à celle d'un cancer de prostate de score de Gleason $6(3+3)$ de $3 \times 2 \mathrm{~mm}$, considéré comme non significatif et habituellement non perçu en IRM clinique en pratique courante. 

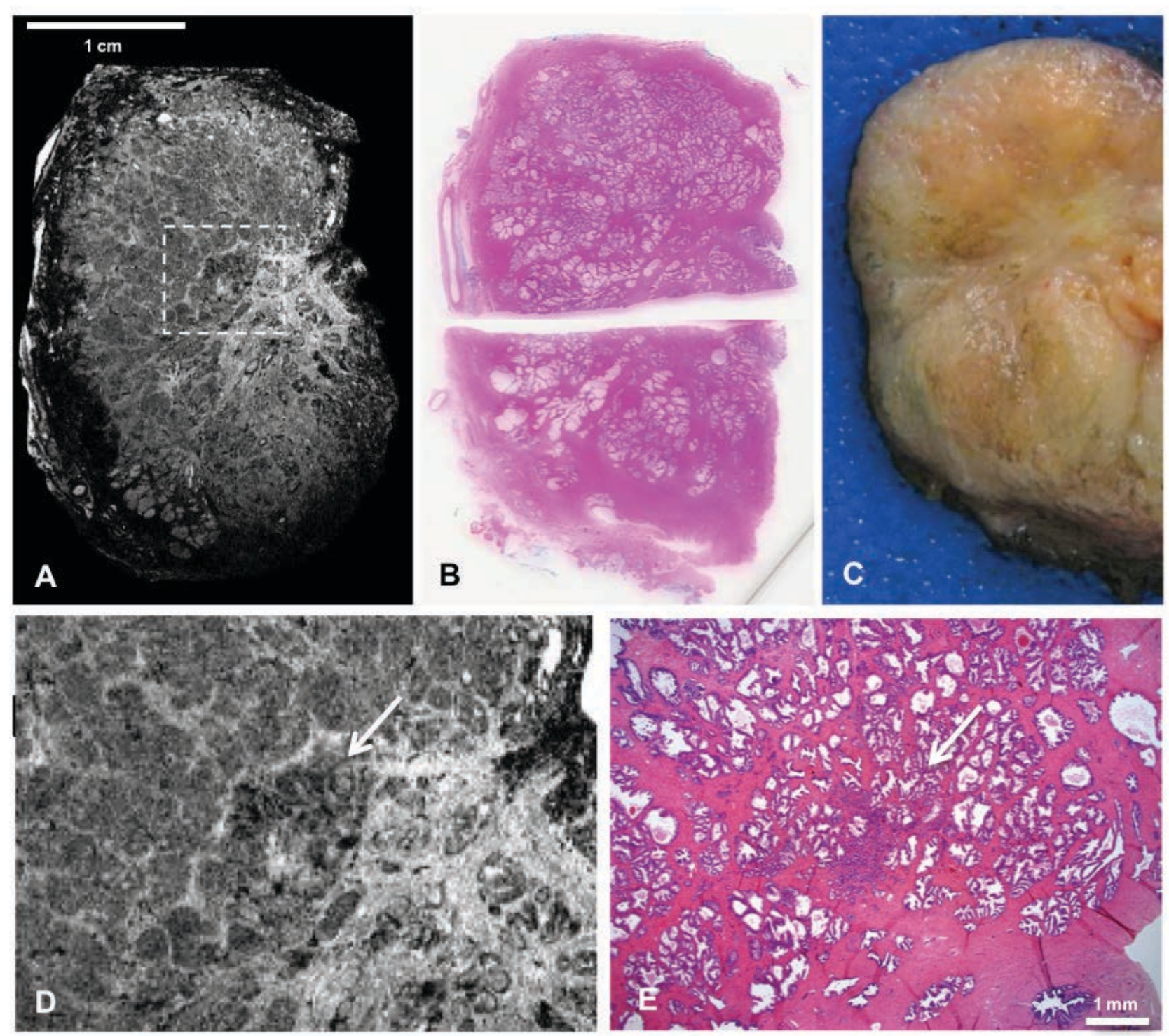

Figure 11 - Image en écho de gradient en IRM 7 Tesla d'un échantillon de prostate issu d'une prostatectomie radicale robot

L'image $(\mathrm{A})$ a été obtenue à une résolution spatiale de $60 \times 60 \times 90 \mu \mathrm{m}^{3}$ en 3h environ après immersion complète dans une solution de $\mathrm{NaCL}$ à $0,9 \%$ et de Gd-DTPA à 1,0\% pendant environ 1,5 avant imagerie (protocole $\mathrm{G}$ ).

Le contraste de l'image délimite l'architecture stromale et glandulaire avec un entrelacement de canaux superposable à l'analyse H\&E correspondante $(B)$. L'échantillonnage de la pièce fraîche $(C)$ avait été réalisé sur une zone maligne suspecte sur la base des biopsies et de l'IRM préopératoire.

L'agrandissement de la zone d'intérêt entre les pointillés (D) met en évidence une hypo-intensité plus élevée dans la zone centrale de la prostate d'environ $3 \times 2 \mathrm{~mm}$ (flèche) qui correspondait à un amas de glandes fusionnées, composés d'un groupe de glandes qui n'étaient plus complètement distinctes du tissu stromale, aux pourtours irréguliers avec raréfaction des lumières, diagnostiqué comme un cancer de prostate de score de Gleason $6(3+3)$ sur la coupe H\&E (E) 
Un échantillon provenant d'un patient avec un cancer de prostate est présenté sur la Figure 12 à la résolution spatiale parmi les plus élevées acquise à 7 Tesla en suivant le protocole G. On observe des tubulo-acini enchâssés dans le stroma (flèche), des coupes de vaisseaux larges et aplaties en périphérie (pointe de flèche) dans le tissu périprostatique correspondant très précisément à couche la plus externe de la capsule prostatique décrite par AK Tewari. L'image permet de suivre les contours crénelés hyperintense d'une partie de l'urothélium délimitant l'urètre (étoile rouge).

L'ensemble des éléments anatomiques identifiables sur l'image IRM 7T (Figure 12A) sont totalement superposables sur la coupe histologique correspondante (Figure 12C).

Dans la zone périphérique postéro-latérale, on note une hypo-intensité plus marquée avec altération de l'organisation tissulaire (ligne en pointillés). Cette zone en IRM présente une diminution de la présence d'acini, avec massification glandulaire traduite par un réhaussement de l'intensité d'hyposignal, un aspect plus flou des microstructure anatomiques avec une perte des délimitations précises et des bordures glandulaires mal-définies. La projection d'intensité maximale à travers une série consécutives d'images IRM en gradient d'écho tridimensionnelle (Figure 12B) confirmait par une majoration de l'hypo-intensité, la disparition de l'architecture tubulo-acineuse suspecte de malignité. L'analyse comparative de la coupe histologique correspondante (Figure 12C) confirmait la présence d'un cancer de prostate de score de Gleason $8(4+4)$. 

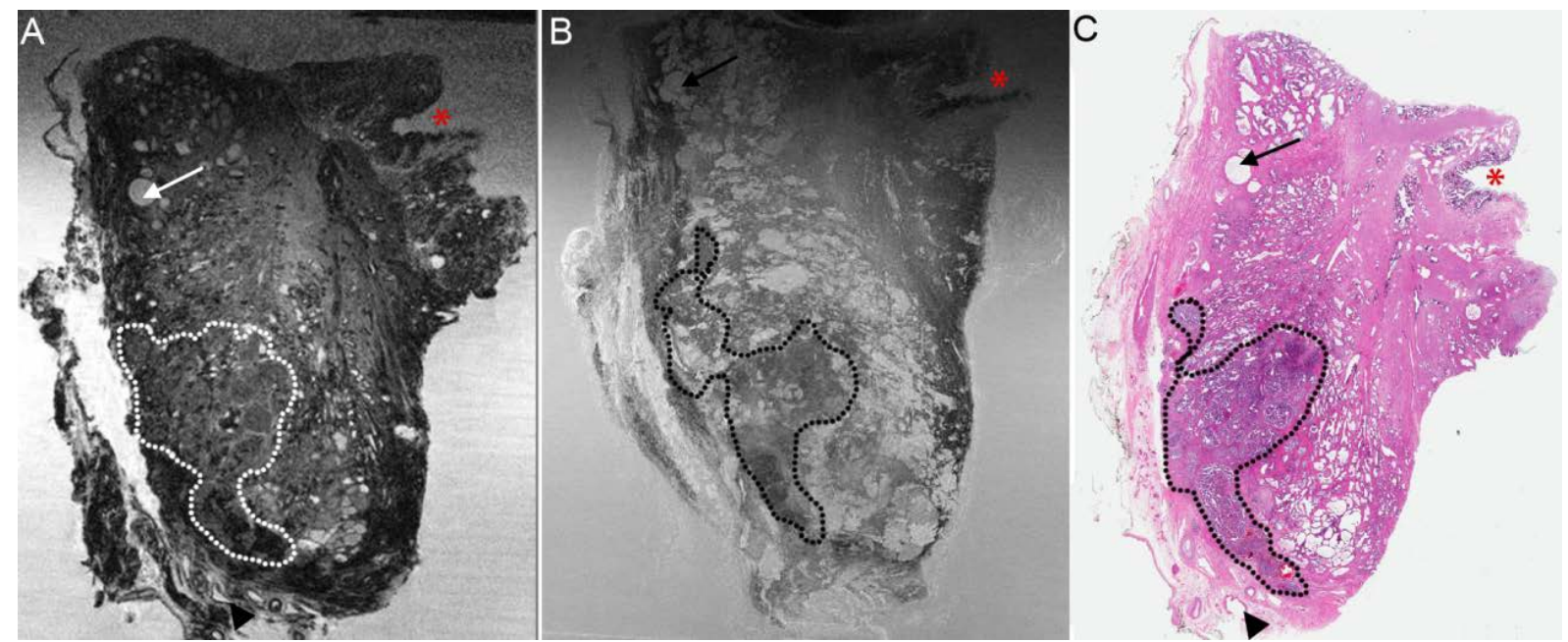

Figure 12 - Image en écho de gradient de 7 Tesla d'un échantillon obtenu à partir de la section de prostatectomie radicale robot avec un cancer de la prostate de haut grade

(A et $B$ ) Les images ont été obtenues à partir d'une acquisition tridimensionnelle à une résolution spatiale de $60 \times 60 \times 90 \mu \mathrm{m} 3$ en environ 3 heures (protocole G). Notez qu'à cette résolution, il est possible de discerner des agrégations spécifiques de cellules (cerclage en pointillé) montrant une tumeur et signalées comme signal faible et confirmées dans la lame colorée $\mathrm{H} \& \mathrm{E}$ correspondante $(\mathrm{C})$. Une projection d'intensité maximale à travers toute la pile d'images produit un ductogramme (B) qui met en évidence la zone de signal faible. La microarchitecture du tissu prostatique, y compris les sécrétions (flèches), les vaisseaux sanguins (tête de flèche) et l'urètre (étoile rouge) étaient facilement identifiable et très nettement délimitées sur cette imagerie de haute résolution. 
Sur la Figure 13, des coupes fraîches contenant un adénocarcinome prostatique provenant de trois échantillons de prostatectomie radicale ont également été obtenues à 7 Tesla avec une résolution de $60 \times 60 \times 90 \mu \mathrm{m}^{3}$ (protocole G). Ces échantillons ont ensuite été soumis pour évaluation histopathologique systématique et se sont révélés être des scores de Gleason $7(4+3)$ (Figure 13C), $8(4+4)$ (Figure 13F) et $9(4+5)$ (Figure 13I). Dans les trois images en 7T de section d'échantillons, la microarchitecture du tissu prostatique est délimitée avec précision, y compris les conduits, les glandes, les vaisseaux sanguins et le stroma. Les glandes bénignes présentaient des sécrétions luminales avec un hypersignal et des bords arrondis relativement lisses et réguliers contrairement à celles des zones tumorales qui révélaient en hyposignal et des canaux souvent mal définis. Les paramètres optimaux ont ainsi pu être atteints pour améliorer l'analyse diagnostique par imagerie du cancer de la prostate. Les images sont tout à fait comparables à celle des coupes histologiques à faible grossissement (X2). De plus, l'obtention de ductogrammes a permis d'augmenter la distinction entre tissu bénin et malin en renforçant l'identification de zone cancéreuse par la majoration d'hyposignal dans les zones de carcinome invasif. L'ensemble des caractéristiques comparatives du tissu prostatique visualisé selon les différents protocoles de paramètres IRM haute résolution à $3 \mathrm{~T}$ et $7 \mathrm{~T}$ pour l'évaluation du cancer de prostate chez l'homme est présenté dans le Tableau 4. 

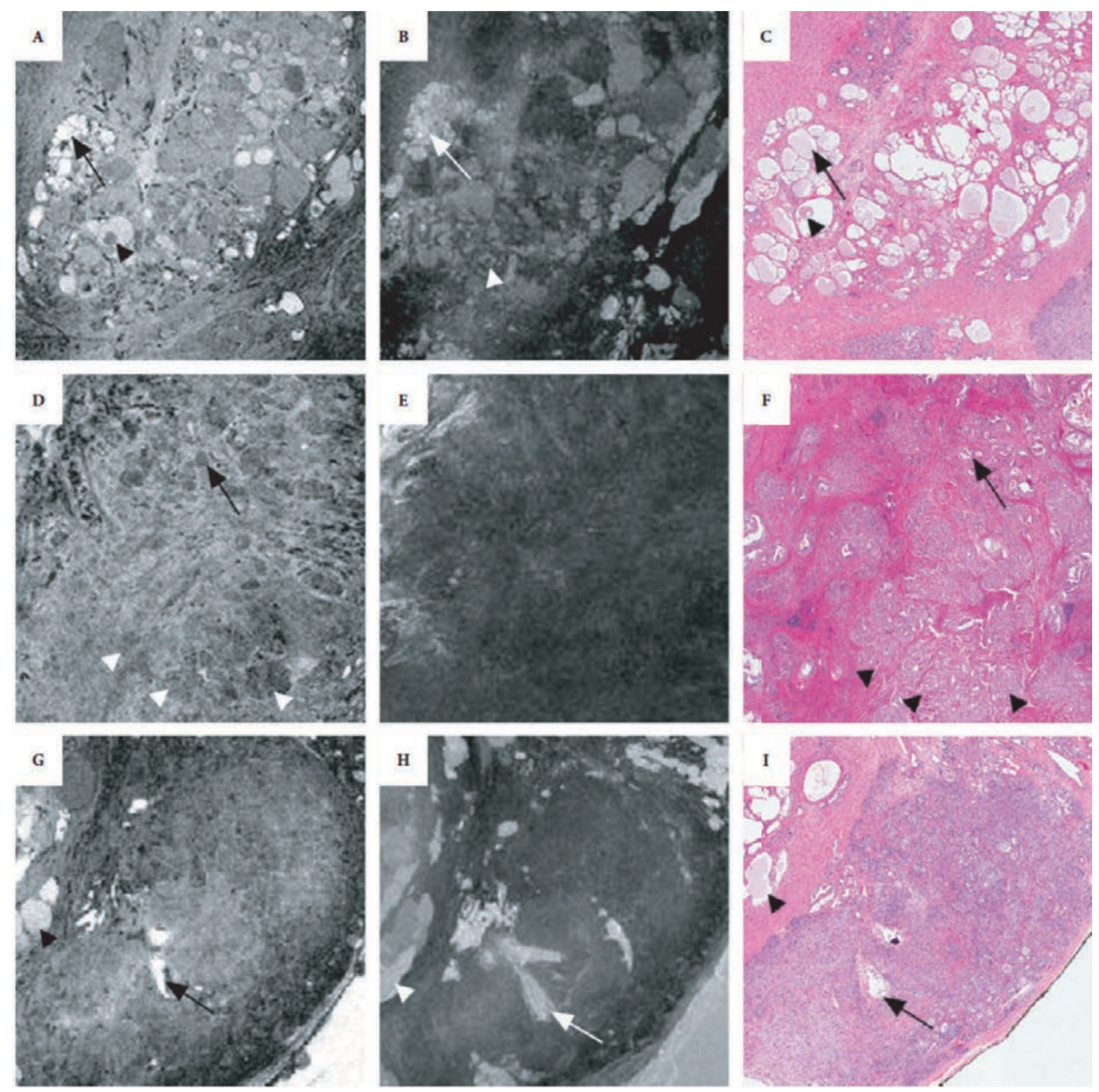

Figure 13 - Série d'images IRM 7T d'échantillons de sections de prostate issues d'une prostatectomie radicale robot chez un même patient révélant des détails microarchitecturaux de glandes bénignes et malignes à haute résolution spatiale de $60 \times 60 \times 90 \mu \mathrm{m} 3$ en 3 heures d'imagerie (protocole G).

(A-C) Coupe passant au niveau d'un nodule hyperplasique bénin. (A) les glandes bénignes avec des sécrétions luminales hyper-intenses (flèches) et des concrétions hypo-intenses (pointes de flèches). (B) Le canal met en évidence ces glandes bénignes et $(C)$ l'image $H$ \& $E$ correspondante confirme le nodule BPH avec des concrétions. Agrandissement : (A-C), zoom numérique $2 x$ des images $1 x$ originales.

(D-F) Section passant au niveau d'un adénocarcinome de score de Gleason $7(4+3)$. (D) Quelques petites glandes hypo-intenses bien délimitées (flèche) sont visibles dans le fond des glandes fusionnées / cribriformes (pointes de flèches). (E) Le ductogramme montre un manque de glandes bénignes hyper-intenses confirmé sur la coupe correspondante histologique $(F)$ avec des petites glandes fusionnées individuelles et plus grandes que les modèles Gleason 3 et 4 respectivement. Grossissement : (D-F), zoom numérique 2x des images $1 x$ originales.

(G-I) Section du spécimen passant au niveau de lésion d'adénocarcinome de score de Gleason $9(4+5)$. (G) Un nodule tumoral hypo-intense avec des glandes bénignes piégées (flèches) et adjacentes (flèches). $(H)$ le canal met en évidence les glandes bénignes hyper-intenses (flèches et pointes de flèche) dans le fond de nodule tumoral hypo-intense et (I) l'image H \& E correspondante confirme le nodule hypo-intense comme Gleason modèle 4 et 5 avec quelques glandes bénignes. Grossissement : (G-I), zoom numérique 2x des images $1 x$ 
Tableau 4 - Caractéristiques comparatives du tissu prostatique utilisant différents protocoles d'imagerie avec l'IRM 3 Tesla et 7 Tesla à haute résolution pour l'évaluation des adénocarcinomes prostatiques humains

\begin{tabular}{|c|c|c|c|}
\hline $\begin{array}{l}\text { Protocole } \\
\text { d'Imagerie }\end{array}$ & Caractéristiques architecturale tissulaire & Analyse du stade tumoral & Caractéristiques intraluminales glandulaire \\
\hline $\begin{array}{l}\text { A, B } \\
\text { (3 Tesla) }\end{array}$ & $\begin{array}{l}\text { Nodules lisses et arrondi d'adénome de prostate (Figure } 7 C \text { ) } \\
\text { Glandes de tailles moyennes et importantes avec un hyposignal } \\
\text { (Figures } 6 \mathrm{~B}, 7 \mathrm{C} \text { ) } \\
\text { Zones mal définies avec un signal hypointense observées dans la } \\
\text { zone périphérique correspondant au cancer de la prostate sur } \\
\text { l'H\&E correspondant (figures } 6 \mathrm{~B}, \mathrm{C} \text { ) }\end{array}$ & $\begin{array}{l}\text { Pas d'évaluation possible pour différencier avec précision le grade de } \\
\text { Gleason }\end{array}$ & $\begin{array}{l}\text { Des concrétions dans la lumière ductale de signal } \\
\text { hyperintense peuvent être visibles mais ne peuvent } \\
\text { pas être localisées avec précision (figure } 6 \mathrm{~B} \text { ) }\end{array}$ \\
\hline $\begin{array}{l}\text { C, D, E } \\
\text { (7 Tesla) }\end{array}$ & $\begin{array}{l}\text { Nodules lisses arrondis mieux définis de l'hyperplasie bénigne } \\
\text { (figure 6D) } \\
\text { Des structures glandulaires plus petites avec et sans hypersignal } \\
\text { dans leur lumière parfois identifiables (figure } 6 D \text { ) } \\
\text { Identification des limites structurelles micro-anatomiques des } \\
\text { vaisseaux et de l'urètre (figures } 6 D \& 8 \text { ) } \\
\text { Zone de signal hypointense superposable à la présence confirmée } \\
\text { de cancer de prostate }\end{array}$ & $\begin{array}{l}\text { Pas d'évaluation possible pour différencier avec précision le grade de } \\
\text { Gleason }\end{array}$ & $\begin{array}{l}\text { Des concrétions en hyposignal apparaissent dans les } \\
\text { lumières en hypersignal des canaux glandulaires } \\
\text { (figure 6D) }\end{array}$ \\
\hline $\begin{array}{l}\text { F, G } \\
\text { (7 Tesla) }\end{array}$ & $\begin{array}{l}\text { Nodules lisses arrondis mieux définis de l'hyperplasie bénigne } \\
\text { (figures 13A, B) } \\
\text { Des structures glandulaires plus petites avec et sans hypersignal } \\
\text { dans leur lumière clairement identifiables } \\
\text { Des glandes fusionnées / cribriformes avec un signal hypointense } \\
\text { délimitables (figure 13D) } \\
\text { La perte globale de l'architecture normale identifiable en cas } \\
\text { d'invasion massive (figure 13D) } \\
\text { Distinction impossible entre une lésion de PIN de haut grade et } \\
\text { une tumeur }\end{array}$ & $\begin{array}{l}\text { Les glandes individuelles et bien développées peuvent être } \\
\text { distinguées (grade } 3 \text { ) des glandes fusionnées et des placards de } \\
\text { cellules glandulaires (grade } 4 \text { ou } 5 \text { ) }\end{array}$ & $\begin{array}{l}\text { Des concrétions en hyposignal franc sont facilement } \\
\text { identifiable dans les lumières en hypersignal des } \\
\text { canaux glandulaires (figure 10A) }\end{array}$ \\
\hline
\end{tabular}




\section{j. Discussion}

Le but de cette étude était d'étudier différents protocoles de paramètres IRM hauterésolution sur un modèle ex vivo de prostatectomie radicale qui pourraient finalement permettre d'obtenir des diagnostics IRM in vivo à une échelle microscopique à condition de pouvoir les transposer. En pratique, deux grands ensembles de protocoles ont été réalisés. Le premier consistait à analyser des glandes entières de prostate en IRM 3T et $7 \mathrm{~T}$ selon des séquences habituelles mais à des résolutions spatiales plus élevées qu'en clinique grâce à l'application d'un résonateur à radiofréquence directement placé contre l'échantillon. Le second utilisait une IRM 7T pour générer un contraste optimum sur des échantillons de prostate de $5 \mathrm{~mm}$ d'épaisseurs, provenant de prostatectomie radicale robot, placés à l'état frais dans un dispositif conçu pour l'étude, selon un plan superposable aux coupes histologiques finales pour permettre une corrélation radio-histologique sans trouble rotatoire.

Il existe un grand nombre de méthodes bien connues pour l'imagerie paramétrique qui ont déjà été appliquées à la prostate, et plusieurs études traitent spécifiquement des comparaisons avec l'histologie de la prostatectomie radicale $[5,43,44]$ pour mieux comprendre comment les paramètres d'imagerie sont liés aux caractéristiques histologiques impliquées. II a été démontré dans une étude de corrélation prospective comparant des résultats d'IRM 1,5T et 3T avec l'anatomopathologie de prostatectomie radicale finale que la détection du cancer sur IRM multiparamétrique était influencée par de multiples facteurs tels que le score de Gleason, le volume, la localisation et l'architecture $(p<0,0001)$ mais pas la force du champ magnétique [45]. À ce jour, une grande partie des attentions sont seulement concentrées sur les résultats histologiques bruts autant que le volume de la tumeur, la macroarchitecture tissulaire 
et l'évaluation du score de Gleason par l'interprétation de l'intensité du signal et du contraste en IRM clinique. Sur la base de nos résultats, nous supposons que, avec une résolution spatiale croissante, en particulier grâce à l'utilisation de champs magnétiques plus élevés tels que 7 T ou 9,4 T [44], il sera possible de caractériser séparément le stroma, les canaux, le système vasculaire, les concrétions ou le carcinome grâce aux temps de relaxation T1 ou T2, aux coefficients de diffusion, autant que par les techniques de rehaussement dynamique de contraste ou l'amélioration des caractéristiques spectrales. À cet égard, nous avons démontré que I'IRM 7T peut délimiter avec précision la microarchitecture du tissu prostatique sur différents protocole T2.

Les résultats obtenus apportaient un haut niveau d'information sur le tissu prostatique au-delà des capacités actuelles des IRM cliniques. L'élévation du champ magnétique s'accompagnait d'un surplus de signal augmentant le niveau de détails visibles permettant l'établissement d'un diagnostic histo-radiologique construit sur l'analyse morphologique structurelle tissulaire permise par un renforcement du signal et contraste. A mesure que les techniques d'imagerie non invasive s'améliorent, l'identification des tissus pourrait devenir ainsi possible. Nous avons trouvé notamment que les glandes bénignes avaient des bords relativement lisses et étaient centrées autour d'une lumière harmonieuse en hypersignal avec parfois des concrétions hypointenses centrales sur les images FLASH. En revanche, les zones tumorales avaient des images d'écho de gradient de signaux hypo-intenses et pouvaient être délimitées distinctement en fournissant une estimation du volume tumoral. 
Fait marquant concernant particulièrement, une divergence a été rapportée dans la délimitation des contours des tumeurs en IRM 3T clinique par rapport à l'analyse histologique finale [46]. Dans notre étude, la plus haute résolution réalisable à 7 Tesla permettait d'établir une correspondance visuelle étroite avec l'histopathologie finale. De sorte que l'augmentation de signal permise par l'augmentation du champ magnétique pourrait apporter plus d'exactitude dans l'appréciation des rapports anatomiques, jusqu'ici sous-estimés par l'imagerie clinique.

La préservation de l'architecture tissulaire témoignait visualiser en IRM hauterésolution semblait corréler à l'absence de lésion maligne. Inversement, sa rupture faisait suspecter sa présence. En IRM haute résolution, une lésion tumorale prostatique se définissait par l'association de plusieurs éléments en hyposignal témoignant d'une structuration proche de l'analyse : prolifération glandulaire anarchique, contours mal-définis de glandes, disparition des lumières tubulaires.

Jusqu'à présent dans le contexte clinique DWI avec une carte ADC est la méthode d'IRM la plus utile pour prédire l'agressivité du cancer [47]. II est important de noter que l'identification d'un cancer cliniquement significatif défini par la présence de Gleason grade 4 ou 5 est essentielle à la prise de décision et aurait pu être estimée dans notre étude par examen des caractéristiques microscopiques de l'architecture glandulaire fournie par 7T IRM. Concrètement, on a pu distinguer des glandes bien définies de petite taille correspondant à des grades de Gleason 3 et des glandes mal définies plus grosses / fusionnées de grade 4 ou 5 de Gleason qui étaient en accord avec les avec les données préliminaires précédemment publiées [48]. 
La technique d'angiogramme appliquée au tissu prostatique permettant la réalisation de l'image représentée en Figure 10C, est la première réalisation de ce type à notre connaissance. Cette imagerie pourrait apporter l'opportunité d'études complémentaires sur le cancer de la prostate si la distorsion micro-anatomique révélée de la vascularisation prostatique est en rapport avec la taille et le grade tumoral. II pourrait également être utile d'étudier si la densité vasculaire dans une tumeur est en corrélation avec le comportement clinique, car la quantification de la densité vasculaire de l'image serait simple.

Les ductogrammes ont amélioré la distinction entre le tissu bénin et le tissu malin et ont permis de caractériser l'agressivité du cancer de prostate en mettant en évidence l'absence de structures canalaires normales (avec leurs sécrétions luminales en hypersignal) dans les zones de carcinome invasif. Cette technique avancée de posttraitement représente un nouveau développement qui n’a pas d'application en clinique. Par une variation de la projection d'intensité maximum permise par une séquence écho de gradient tridimensionnel au travers d'une pile d'images, on rapportait une variation de signal complémentaire avec un hypersignal intense révélant le cheminement sinueux des acini et canaux spécifiques du tissu glandulaire. En présence de cancer laminant la construction harmonieuse des canaux, on notait alors par cette technique un hyposignal renforcée dans la zone la distorsion tissulaire cancéreuse. Cette technique se rapproche du principe de la galactographie utilisée utilisé en sénologie en association des mammographies pour observer la distorsion des canaux mammaires et aider au diagnostic des papillomes intra-canalaires [49].

Nous avons déterminé que l'échelle de longueur pertinente pour la caractérisation correcte du cancer de prostate dans le contexte des caractéristiques normales de la 
prostate peut être proche d'une résolution spatiale de $60 \times 60 \times 90 \mu \mathrm{m}^{3}$. À cette résolution, les caractéristiques sont comparables à l'histopathologie (X 2).

Notre objectif dans cette étude était d'identifier un phénotype radiologique du cancer de prostate qui pourrait être utile à l'avenir. Bien que le nombre relativement faible de spécimens dans notre analyse limite nos conclusions, il est raisonnable de supposer qu'étant donné le progrès rapide des techniques d'imagerie radiologique, les images cliniques in vivo commenceront à s'approcher de la frontière histologique dans un avenir relativement proche. II semble évident à partir du présent travail que les radiologues évalueront des formes de contraste d'image considérablement différentes de celles qui sont maintenant acceptées afin d'interpréter correctement les images à haute résolution de la prostate.

Nos analyses ont souligné aussi certaines limites de la haute résolution. Notamment, l'augmentation de l'intensité de l'aimant s'accompagnait aussi d'apparition de nouvelles zones de perte de signal parasitant l'interprétation de l'imagerie. Ces pertes pourraient être expliquées par le mécanisme de susceptibilité magnétique des tissus qui correspond à la propre aimantation interne tissulaire induite par un champ magnétique B0. Ce phénomène de susceptibilité [50] constitue un frein à la performance de l'imagerie haute résolution. II est source de distorsion du champ magnétique entraînant phasage et déphasage de fréquences localisés et conduit à la perte de signal constatée qui augmente de façon linéaire à l'intensité du champ magnétique.

L'extrapolation de l'ensemble de ces résultats en situation clinique in vivo doit être prudente. Le protocole était conçu pour valider le principe de proportionnalité entre la 
haute résolution et l'augmentation du niveau d'information aidant à l'établissement du diagnostic de cancer de prostate. Ce modèle expérimental ne tenait pas compte de l'environnement anatomique de la prostate in situ dont la profondeur dans le pelvis entraînerait un RSB de moins bonne qualité et une potentielle perte d'information. 


\section{Modèle et Travaux expérimentaux 2}

II s'agit d'une étude transversale prospective non randomisée de performance diagnostique, comparant les résultats de l'IRM biparamétrique 7Tesla (test index) à l'histologie (test de référence) selon un modèle expérimental d'imagerie sur pièces opératoires de prostatectomie radicales robot pour cancer de prostate.

\section{a. Objectifs principal \& secondaires}

L'objectif était d'évaluer la performance diagnostique de l'IRM 7T biparamétrique (T2W, DWI) pour différencier le tissu malin du tissu sain sur des échantillons de prostate de patients opérés de prostatectomie en optimisant les paramètres de séquences IRM issus des travaux expérimentaux 1. Le test de référence standard était le résultat d'analyse histologique finale de la pièce opératoire.

Secondairement, les analyses réalisées comparaient la reproductibilité interobservateur et portaient sur la reconnaissance des microstructures anatomiques identifiées de l'imagerie de pièce fraîche par l'IRM 7T biparamétrique, en se basant sur les résultats lésionnels anatomopathologiques finaux.

\section{b. Cohorte d'étude}

Tous les patients successifs opérés pour un cancer localisé de la prostate par prostatectomie radicale élargie robot dans un seul centre étaient éligibles. La cohorte se compose de $n=18$ prostates complètes de patients consentants pris en charge dans le département d'urologie de Icahn. La durée de la période d'inclusion était de 9 mois. L'ensemble ce ces patients avait bénéficié d'un bilan préopératoire conventionnel incluant le dosage de leur PSA, au minimum une série de biopsies prostatique et une IRM de prostate multicentrique avec relecture systématique des images dans le centre 
de référence par une équipe d'uro-radiologiste dédiée. N'ont pu être retenus dans l'étude, les patients pour lesquels, des antécédents chirurgicaux ou de radiothérapie de prostate existaient. Ont été exclus les sujets dont les analyses d'imagerie des échantillons n'ont pu être réalisées par demande spécifique de l'opérateur, souhaitant privilégier l'analyse histologique standard sans délai d'imagerie intermédiaire, ou pour des raisons de dysfonctionnement technique des séquences IRM prévus.

\section{c. Consentement \& cadre réglementaire}

Après explication et récupération de leur consentement éclairé écrit (GCO\#14-0417) signé au préalable de leur intervention, les patients étaient inclus. Cette étude a été revue et approuvée par un comité de révision d'éthique institutionnel de Mount Sinai, (Study ID\# : HSM\#14-00056 - IRB approved). Ces travaux ont été menés chez des patients malades volontaires dans le cadre d'un programme de recherches plus largement étendu qui visaient à tester des nouvelles modalités de techniques d'imagerie avancées sur des échantillons humains de patients atteints de cancer de prostate. L'enjeu présenté aux candidats volontaires dans ce cadre de recherche était d'explorer de nouvelles modalités technologiques pour distinguer les cellules cancéreuses du reste des structures qui intra- et extra-prostatiques, pour identifier les nerfs érecteurs sinueux sur la capsule et pour en dissocier visuellement les vaisseaux associés.

L'étude a été ouverte une pour une durée de 1 an et demi de 2015 à 2016 sous la responsabilité de l'investigateur principal, Ashutosh Tewari, MD, chef de service d'Urologie de Mount Sinai Hospital, NYC, USA. Le consentement précisait que les tests étaient réalisés après chirurgie de prostatectomie radicale robot. L'imagerie concernait exclusivement le spécimen de prostate ex vivo. Aucun test n'était réalisé 
sur les patients eux-mêmes. II ne s'agissait que d'un seul examen d'imagerie par échantillon de prostate dès leur résection. Aucun médicament, ni aucun dispositif médical n'était utilisé pour cette étude. Consentir à cette étude ne prolongeait pas le séjour hospitalier, n'impliquait pas de visites médicales, ni d'hospitalisations ou de consultations externes supplémentaires. Un suivi standard était prévu comme toute autre personne qui bénéficiait de cette opération et qui n'était pas dans l'étude.

Aucun bénéfice individuel n'était attendu. Le fruit des recherches réalisées avait pour ambition d'aider à permettre une meilleure prise en charge peropératoire ultérieures si les résultats étaient satisfaisants et reproductibles. La signature des consentements engageait, par responsabilité, les patients à bien vouloir partager leurs informations médicales en lien avec leur maladie pendant une durée de 5 ans par l'intermédiaire de l'équipe de recherche à partir des dossiers médicaux patients.

Ils étaient prévenus des risques faibles inhérents à l'étude de perte d'informations protégées ou du risque d'altération ou de perte de leur échantillon prostatique du fait des manipulations ou du circuit de circulation de leur pièce opératoire. À tout moment, les patients pouvaient sortir de l'étude de leur plein gré sur simple demande signée sans devoir le motiver par une quelconque explication. Leur retrait était sans risque pour eux de rupture de soins ou de toute autre conséquence négative à risque de préjudice. Les patients, par leur signature, conformément à la loi fédérale sur la transférabilité et la responsabilité en matière d'assurance maladie (Health Insurance Portability and Accountability Act (HIPAA)), acceptait que les professionnels de santé concernés puissent avoir accès aux données médicales personnelles et les partager dans le cadre de l'étude.

Leur compagnie d'assurance devait être responsable de tous les coûts des soins cliniques standard pour le diagnostic et le traitement de leur état. Aucun coût ni 
paiement supplémentaire n'était prévu pour les patients ou leur assurance pour participer à cette étude. Les fonds pour mener cette recherche étaient apportés entièrement par le département d'Urologie de Icahn School of Medicine de Mont Sinai.

\section{d. Préparation, manipulations \& suivi des échantillons prostatiques pour imagerie}

Les patients bénéficiaient tous d'une prostatectomie radicale robotique selon la technique chirurgicale standard avec préservation neuro-vasculaire selon évaluation des risques individuels [51]. L'ensemble des interventions était mené au bloc opératoire de Mount Sinai Hospital, au 1468 Madison Ave, NYC, USA, par un seul opérateur chirurgien-urologue, Ashutosh Tewari, MD. Le protocole opératoire, l'identitovigilance et l'horodatage des pièces fraîches de prostatectomie étaient réalisés suivant les mêmes procédures décrites dans les travaux expérimentaux 1 (cf. IV d). La prostate fraîche placée dans un sac chirurgical puis extraite au travers d'un anneau rétracteur de plaies de taille $S$ de type Alexis ${ }^{\circledR}$ (Applied Medical, CA, USA), était réceptionnée directement en salle par le coordonnateur de recherche.

Le conditionnement de la pièce avait lieu secondairement dans la continuité en salle de «Frozen section » de Mount Sinai Hospital au-dessus du bloc opératoire. La pièce opératoire alors était mesurée, photographiée puis placée intégralement, y compris les vésicules séminales, dans un récipient en plastique avec un bouchon vissé hermétiquement. Son diamètre était de $5 \mathrm{~cm}$, adapté à la fois à celle du spécimen, limitant les compressions tissulaires par écrasement, et à celle du trou de l'aimant de l'IRM dans lequel il devait ensuite rentrer. Du papier-tissu pouvait être ajouté en fonction de la taille de la prostate pour immobiliser la pièce et limiter les risques d'artefact de mouvements induits par les vibrations de l'IRM en marche. 
Les pièces étaient orientées dans le récipient pour éviter des erreurs de lecture. Un repère, composé d'un tube en plastique long accolé à un second plus court, confectionné à partir de 2 cylindres de seringue de $2 c c$, était glissé conventionnellement le long du côté droit de chaque prostate. Le tube le plus long s'étendait de la base à l'apex et le plus court de la base à la portion moyenne de la glande. Ce repère, visible en hyposignal franc à l'IRM, servait de témoin systématique pour l'orientation de la pièce dans les 3 dimensions.

Le récipient en plastique était finalement rempli de sérum physiologique ou de liquide électronique Fluorinert ${ }^{\mathrm{TM}}$ FC-770 (3M, St.Paul, MN, USA). Ce fluide avait été choisi pour ses qualités d'isolant électrique dans le but d'améliorer les contrastes IRM. Avant la fermeture par le couvercle, les bulles d'air emprisonnés dans le montage étaient chassées par de légers mouvements de vibration manuels. Le récipient une fois recouvert de son couvercle vissé, pouvait être enveloppé d'un film en latex complémentaire - produit à base de gant chirurgical - pour assurer l'étanchéité complète du récipient, placé au cœur de l'IRM, pendant toute la durée d'imagerie. La Figure 14 représente l'ensemble de la préparation. 


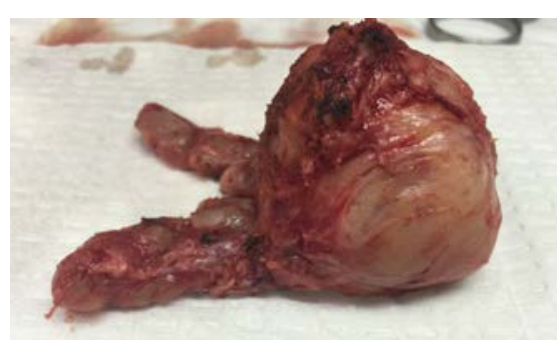

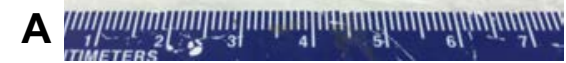

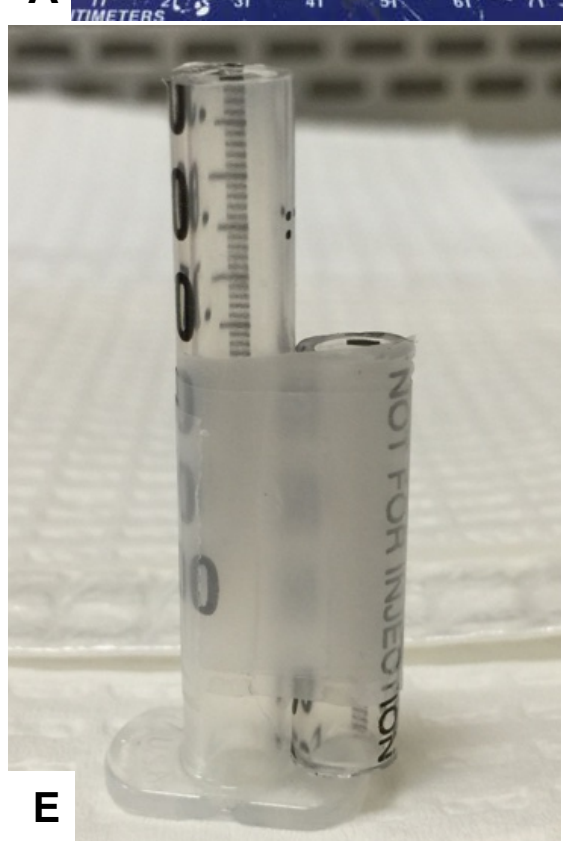

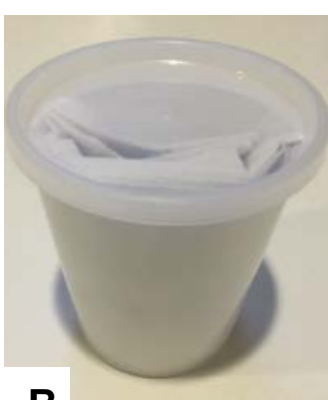

B

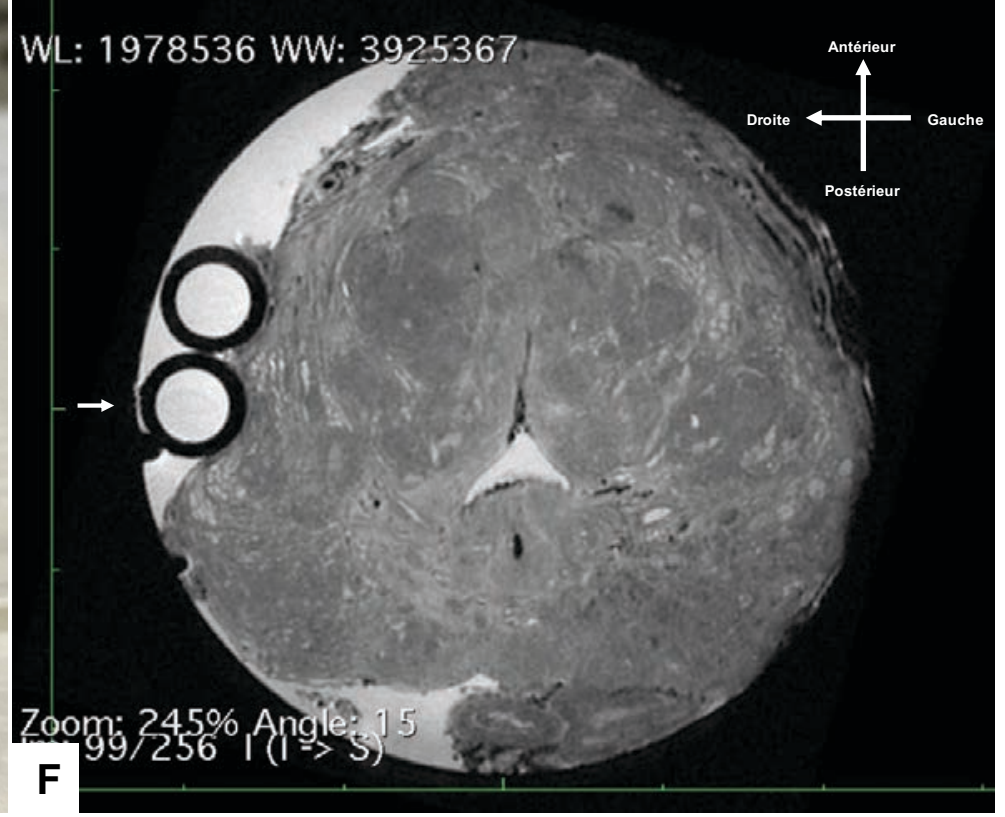

Figure 14 - Préparation \& orientation de l'échantillon de prostate

(A) La pièce fraîche était mesurée dans ces 3 axes. (B-D) On utilisait un récipient hermétique en plastique de $5 \mathrm{~cm}$ de diamètre pour contenir la glande prostatique entière. Le conteneur était rempli de sérum physiologique ou de Fluorinert ${ }^{\mathrm{TM}}$ FC-770 (3M, St.Paul, MN, USA). Du papier tissu était placé de part et d'autre de la prostatectomie pour limiter ses déplacements pendant l'imagerie. On reportait l'orientation sur le couvercle pour aider au positionnement dans le trou de l'IRM. (E) Un repère en plastique, composé de 2 tubes cylindriques de $5 \mathrm{~mm}$ de diamètre liés l'un à l'autre était confectionné pour chaque imagerie de spécimen. II était systématiquement placé à la droite de la prostate, sur toute sa longueur. Le tube le plus long atteignait l'apex et le plus court la portion moyenne de la glande. (F) Imagerie en coupe IRM 7T d'un échantillon orienté grâce au repère visible par 2 cercles en hyposignal (flèche blanche). La présence des 2 cylindres permet d'indiquer le niveau de coupe (ici entre la base et la portion moyenne) et le côté droit de la prostate. 


\section{e. Protocoles d'imagerie IRM 7T haute résolution}

Nous avons utilisé un système Bruker Biospec ${ }^{\circledR}$ 70/30 avec un scanner micro-IR 7T (Bruker Biospin, Billerica, MA, USA) pour réaliser l'imagerie IRM 7T des pièces fraîches complètes orientées après prostatectomie radicale. L'acquisition était réalisée grâce à une antenne à 4 canaux conçue initialement pour l'imagerie cérébrale de souris (Figure 15). Un fois le récipient placé dans l'antenne, après balayage du localisateur, l'échantillon était aligné manuellement dans le tunnel de l'IRM pour obtenir une distorsion optimale du signal.
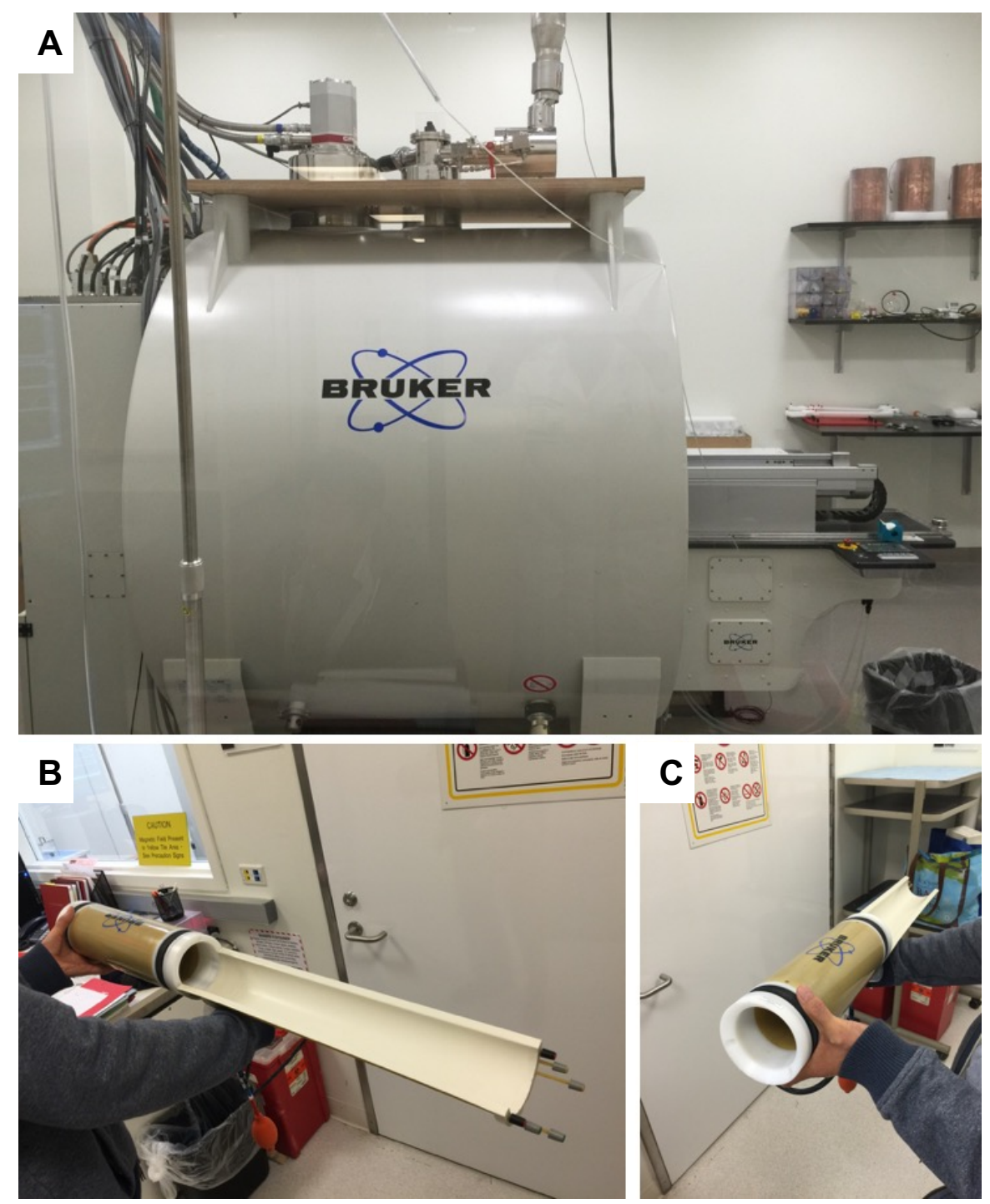

Figure 15 - Dispositif complet IRM 7T expérimental

(A) Système Bruker Biospec ${ }^{\circledR}$ 70/30 avec un scanner micro-IR 7T (Bruker Biospin, Billerica, MA, USA). (B-C) L'acquisition était réalisée grâce à une antenne à 4 canaux conçue initialement pour l'imagerie cérébrale de souris. 
Plusieurs protocoles IRM ont été initiés associant FLASH_3Dslab (T1) - TurboRARE3D (T2) - EPI-DTI (DWI). Les séquences T1 et principalement T2 ont été optimisée pour atteindre la résolution la plus proche de notre objectif défini par nos travaux préliminaires. Notre série ajoutait des gradients de diffusion dans la phase préparatoire des séquences d'imagerie. La séquence d'écho planar - écho de spin a été retenue pour limiter les artéfacts de mouvements. On a eu recours à une acquisition parallèle pour accroitre la qualité des images de diffusion en réduisant la durée de la séquence, le temps d'écho et certains artéfacts. Le protocole typique d'imagerie pondérée par la diffusion commençait par la production d'une image de base b0 obtenue avec tous les gradients de diffusion sensibles désactivés. Les gradients de diffusion étaient ensuite appliqués individuellement et dans différentes combinaisons pour produire un ensemble d'images de source sensibilisées à la diffusion dans diverses directions.

Les séquences étaient répétées en appliquant les gradients de diffusion dans au moins 3 directions de l'espace. Pour quantifier la diffusion, des images de TRACES-DW (images pondérées en diffusion globale) étaient calculées à partir de la séquence de diffusion (DTI) et obtenues en faisant la moyenne des trois cartographies ADC dans les 3 directions $x$, y et $z$. En utilisant la valeur moyenne des TRACES-DW (Dxx + Dyy $+D z z) / 3$, on réduisait la diffusivité multidirectionnelle à chaque point en un nombre unique pouvant considérer comme un coefficient de diffusion apparent consolidé $(A D C)$. La cartographie $A D C$ à orientation-dépendante correspondait donc à un ensemble d'images dérivés du TRACES-DW pour lesquels les effets T2 étaient soustraites. Les images TRACES-DW possédant une pondération T2 très importante, des lésions avec des valeurs T2 très courtes ou très longues aurait pu les faire apparaître faussement en hypo- ou en hypersignal. Les effets T2 étaient donc éliminer 
mathématiquement de l'image DW, créant une image paramétrique pure d'ADC. L'unité des images de TRACES-DW était de l'ordre de 1,0 X 10-3 mm²/s. L'ensemble des protocoles d'imagerie est présenté dans le Tableau 5. 
Tableau 5 - Protocoles de paramètres d'acquisition d'imagerie IRM 7T de séquences T1W, T2W, DW

\begin{tabular}{|c|c|c|c|c|c|c|c|c|c|c|c|c|c|c|}
\hline $\begin{array}{l}\text { Séquence } \\
\text { IRM }\end{array}$ & $\begin{array}{l}\text { Nombre } \\
\text { d'images }\end{array}$ & Pulse Seq & $A C Q$ & $\mathrm{TR}(\mathrm{ms})$ & TE (ms) & $\mathrm{CV}(\mathrm{cm})$ & $\begin{array}{c}\text { Matrice } \\
\text { Acquisition } \\
(\mathrm{cm})\end{array}$ & Flip Angle & $\begin{array}{l}\text { Épaisseur } \\
\text { Coupe } \\
(\mathrm{mm})\end{array}$ & B Value & $\begin{array}{c}\text { NEX (or } \\
\text { Av) }\end{array}$ & $\begin{array}{l}\text { ETL (or } \\
\text { Rare } \\
\text { factor) }\end{array}$ & $\begin{array}{c}\text { Temps } \\
\text { (hr:min:sec) }\end{array}$ & $\begin{array}{l}\text { Résolution } \\
\qquad(\mu \mathrm{m} 3)\end{array}$ \\
\hline T1W & 32 & Flash & $3 \mathrm{D}$ & 36,4 & 6,1 & 5 & $256 / 0 / 0 / 256$ & 15 & 1.563 & I & 1 & 1 & 00:16:09 & $195 \times 195 \times 1563$ \\
\hline T1W & 64 & Flash & $3 \mathrm{D}$ & 36,4 & 6,1 & 5 & $256 / 0 / 0 / 256$ & 30 & 0,781 & I & 1 & 1 & 00:10:09 & $195 \times 195 \times 781$ \\
\hline T1W & 256 & Flash & $3 \mathrm{D}$ & 36,4 & 15 & 5 & $256 / 0 / 0 / 256$ & 15 & 0,195 & I & 7 & 1 & 04:39:41 & $195 \times 195 \times 195$ \\
\hline T1W & 256 & Flash & $3 \mathrm{D}$ & 36,4 & 15 & 5 & $256 / 0 / 0 / 256$ & 15 & 0,195 & I & 3 & 1 & $01: 59: 32$ & $195 \times 195 \times 195$ \\
\hline $\mathrm{T} 2 \mathrm{~W}$ & 1 & RARE & $2 \mathrm{D}$ & 2000 & 127.28 & 5 & $504 / 0 / 0 / 1024$ & 180 & 0.75 & I & 16 & 24 & $00: 41: 51$ & $107 \times 107 \times 750$ \\
\hline $\mathrm{T} 2 \mathrm{~W}$ & 18 & RARE & $2 \mathrm{D}$ & 3878 & 92.57 & 5 & $504 / 0 / 0 / 1024$ & 180 & 0.750 & I & 40 & 18 & $01: 17: 47$ & $107 \times 107 \times 750$ \\
\hline $\mathrm{T} 2 \mathrm{~W}$ & 32 & TurboRARE & $3 \mathrm{D}$ & 1500 & 70 & 5 & $256 / 0 / 0 / 256$ & 180 & 2.656 & I & 1 & 16 & $00: 27: 27$ & $195 \times 195 \times 2656$ \\
\hline $\mathrm{T} 2 \mathrm{~W}$ & 32 & TurboRARE & $3 \mathrm{D}$ & 2000 & 43.4 & 5 & $256 / 0 / 0 / 256$ & 180 & 2.188 & I & 1 & 16 & $00: 50: 05$ & $195 \times 195 \times 2188$ \\
\hline $\mathrm{T} 2 \mathrm{~W}$ & 32 & TurboRARE & $3 \mathrm{D}$ & 1500 & 43.4 & 5 & $256 / 0 / 0 / 256$ & 180 & 2.188 & I & 3 & 16 & 00:38:48 & $195 \times 195 \times 2188$ \\
\hline $\mathrm{T} 2 \mathrm{~W}$ & 64 & TurboRARE & $3 \mathrm{D}$ & 1500 & 70 & 5 & $256 / 0 / 0 / 256$ & 180 & 0.938 & 1 & 4 & 16 & 01:42:48 & $195 \times 195 \times 938$ \\
\hline $\mathrm{T} 2 \mathrm{~W}$ & 64 & TurboRARE & $3 \mathrm{D}$ & 1500 & 70 & 6 & $256 / 0 / 0 / 256$ & 180 & 1.250 & I & 1 & 16 & ND & $273 \times 273 \times 1250$ \\
\hline $\mathrm{T} 2 \mathrm{~W}$ & 64 & TurboRARE & $3 \mathrm{D}$ & 1500 & 70 & 5 & $256 / 0 / 0 / 256$ & 180 & 1.094 & I & 1 & 16 & ND & $234 \times 234 \times 1094$ \\
\hline $\mathrm{T} 2 \mathrm{~W}$ & 64 & TurboRARE & $3 \mathrm{D}$ & 1500 & 70 & 5 & $384 / 0 / 0 / 384$ & 180 & 0.938 & 1 & 1 & 16 & 01:09:26 & $130 \times 130 \times 938$ \\
\hline $\mathrm{T} 2 \mathrm{~W}$ & 64 & TurboRARE & $3 \mathrm{D}$ & 1500 & 100 & 5 & $256 / 0 / 0 / 256$ & 180 & 1.094 & I & 1 & 16 & $00: 25: 53$ & $195 \times 195 \times 1094$ \\
\hline $\mathrm{T} 2 \mathrm{~W}$ & 256 & TurboRARE & $3 \mathrm{D}$ & 1500 & 70 & 5 & $256 / 0 / 0 / 256$ & 180 & 0.195 & I & 2 & 16 & $04: 25: 12$ & $195 \times 195 \times 195$ \\
\hline $\mathrm{T} 2 \mathrm{~W}$ & 256 & TurboRARE & $3 \mathrm{D}$ & 1500 & 43.4 & 5 & $256 / 0 / 0 / 256$ & 180 & 0.195 & I & 4 & 16 & 06:50:03 & $195 \times 195 \times 195$ \\
\hline $\mathrm{T} 2 \mathrm{~W}$ & 256 & TurboRARE & $3 \mathrm{D}$ & 1800 & 80 & 5 & $384 / 0 / 0 / 384$ & 180 & 0.195 & I & 4 & 16 & $12: 19: 10$ & $130 \times 130 \times 195$ \\
\hline DTI, DW & $32-28$ & PGSE-EPI & $2 \mathrm{D}$ & 3000 & 79 & 5 & $256 / 0 / 0 / 256$ & 180 & 0.5 & $\begin{array}{c}1250,16 \\
\text { directions } \\
2 b_{0}\end{array}$ & 2 & 16 & 03:00:00 & $390 \times 390 \times 500$ \\
\hline
\end{tabular}




\section{f. Analyses de l'imagerie}

Analyse morphologique qualitative. L'évaluation de la qualité des images d'échantillons de prostatectomie obtenues a été réalisée sur les séquences pondérées en T2W en 256 images, sur la diffusion (DWI) et la cartographie ADC. L'analyse a été menée en aveugle par 2 relecteurs indépendants. Le relecteur \#1 était urologue et le relecteur \#2 était uro-radiologue avec plus de 20 ans d'expérience en IRM. Aucun des deux relecteurs n'avait connaissance des données cliniques des patients, des comptes rendus IRM préopératoires ni des résultats anatomo-pathologiques finaux au moment de leur relecture. L'évaluation portait sur différents critères de jugement prédéfinis par séquences. On utilisait une échelle de "Likert » entre 1 et 5 niveaux pour caractériser les critères d'étude et nuancer le degré d'interprétation.

L'analyse en T2W reposait sur l'analyse de 3 critères morphologiques de microanatomie prostatique. Le premier critère correspondait à l'anatomie zonale, la délimitation de l'urètre et des bords de la prostate d'une ; le second à la délimitation des vésicules séminales et des canaux éjaculateurs ; le troisième à la délimitation des nodules d'adénome de prostate. L'échelle permettait de caractériser les critères de la façon suivante : 1 - non identifié ; 2 - diagnostic difficile ; 3 - diagnostic satisfaisant ; 4 - diagnostic facile ; 5 - diagnostic excellent.

L'analyse de la diffusion (DWI) portait sur 3 autres critères : distorsion, artéfacts et netteté d'image. L'échelle de score était la suivante : 1 - extrêmement médiocre ; 2 médiocre ; 3 -satisfaisant ; 4 -bon ; 5 - excellent. L'analyse de la cartographie ADC ne portait que sur un seul critère d'appréciation globale de la qualité sur 5 points. Le score maximum par relecteur pour l'imagerie d'un patient était donc sur 15 pour les 
images pondérées en T2W, sur 15 pour celles de diffusion (DWI) et sur 5 pour les cartographies ADC. Le score individuel d'évaluation qualitative morphologique portait donc au total sur 35 points. Un exemple de feuille d'analyse qualitative morphologique d'imagerie IRM 7T biparamétriques par patient est représentée dans le Tableau 6.

Tableau 6 - Évaluation morphologique IRM 7T biparamétrique (T2W : visualisation des structures anatomiques prostatique ; DWI (b16 ; b1250) ; cartographie ADC)

\begin{tabular}{|c|c|}
\hline & Relecteur \#2 \\
\hline & 1 à 5 \\
\hline \multicolumn{2}{|c|}{$\mathrm{T} 2 \mathrm{~W}$} \\
\hline \multicolumn{2}{|c|}{ Anatomie zonale / Urètre et délimitation du bord de la prostate } \\
\hline \multicolumn{2}{|c|}{ Délimitation des vésicules séminales et des canaux éjaculateurs } \\
\hline \multicolumn{2}{|c|}{ Délimitation des nodules d'adénome de prostate } \\
\hline Sous-TOTAL Score T2W & $/ 15$ \\
\hline
\end{tabular}

\begin{tabular}{|c|c|c|}
\hline \multicolumn{2}{|c|}{ DWI } & \\
\hline Distorsion & & \\
\hline Artéfact & & \\
\hline Netteté de l'image & $/ 15$ & $1 / 15$ \\
\hline Sous-TOTAL Score DWI
\end{tabular}

\begin{tabular}{|c|c|c|}
\hline \multicolumn{2}{|c|}{ ADC } & \\
\hline Qualité d'image globale & & 15 \\
\hline Sous-TOTAL Score ADC & $/ 5$ & \\
\hline
\end{tabular}

\begin{tabular}{|c|c|c|}
\hline \multicolumn{3}{|c|}{ ADC } \\
\hline $\begin{array}{c}\text { TOTAL Score individuel d'évaluation qualitative } \\
\text { morphologique }\end{array}$ & $/ 35$ & $/ 35$ \\
\hline
\end{tabular}


Analyse de susceptibilité lésionnelle tumorale. Au cours de la même séance, les images étaient relues une seconde fois uniquement par le relecteur \#2 pour détecter, localiser et décrire les foyers suspects de cancer de prostate. L'analyse portait pour chaque patient sur l'ensemble des séquences disponibles d'imagerie IRM biparamétrique 7T qui avait été obtenue pour chaque pièce fraîche de prostatectomie radicale. L'interprétation se faisait simultanément sur les mêmes niveaux de coupes de séquences différentes grâce à un logiciel de relecture (OsiriX DICOM viewier ; Pixmeo, Genève Suisse) capable d'afficher toutes les séries de façon synchronisée, et de mettre un pointeur sur toutes les séries en temps réel pour analyser rapidement la sémiologie de la zone suspecte. Un schéma de lecture était respecté, passant en revue de façon indépendante les trois principaux compartiments prostatiques comme suit: zone périphérique $(\mathrm{ZP})$, zone de transition $(\mathrm{ZT})$ et stroma fibromusculaire antérieur (SFMA).

En cas de lésion suspecte détectée, chaque anomalie était reportée sur schéma de sectorisation standardisée dans l'un des 27 secteurs préconisés par conférence de consensus [52], et évaluée selon un score standardisé par séquence. Un maximum de 3 lésions distinctes parmi les plus grandes étaient identifiées. Les lésions étaient classées pour chaque patient de 1 à 3, par ordre décroissant de taille exprimée en mm et mesurée selon leur plus grand axe sur les coupes transversales en T2W. Le score lésionnel était évalué comme suit en T2W (0 : isointense, 1 : hypo-intense) ; en DWI de valeur b élevée $(0$ : isointense, 1 : hyperintense) ; sur la cartographie ADC (0 : isointense, $1:$ hypointense). Pour chaque anomalie de signal établie sur chacune des séquences, un score de perceptibilité complétait l'interprétation. II était basé sur le contraste entre la lésion suspecte et le tissu prostatique sain de voisinage sur chacune 
des séquences en T2W, en DWI $\left(b=1250 \mathrm{~s} / \mathrm{mm}^{2}\right)$ et sur la cartographie ADC. On scorait selon une échelle de "Likert" de 1 à 5 niveaux pour caractériser les anomalies (1 - à peine visible ; 2 - assez visible ; 3 - moyennement visible ; 4 - bien vue ; 5 - très bien et nettement définie). Enfin, un score de perceptibilité global était calculé en réalisant la moyenne des scores de perceptibilité par séquence. Une fiche d'évaluation lésionnelle individuelle est représentée en Figure 16.

Lesion assessment -mark the tumor in the prostatic map
\begin{tabular}{|l|c|c|c|}
\hline $\begin{array}{l}\text { T2W Se: } \\
\text { Lesion \# }\end{array}$ & 1 & 2 & 3 \\
\hline Size mm $\times \mathrm{mm}$ & & & \\
\hline $\begin{array}{l}\text { No lesion (0) } \\
\text { Lesion (1) }\end{array}$ & & & \\
\hline $\begin{array}{l}\text { If lesion - assess } \\
\text { lesion conspicuity } \\
(1-5)\end{array}$ & & & \\
\hline
\end{tabular}

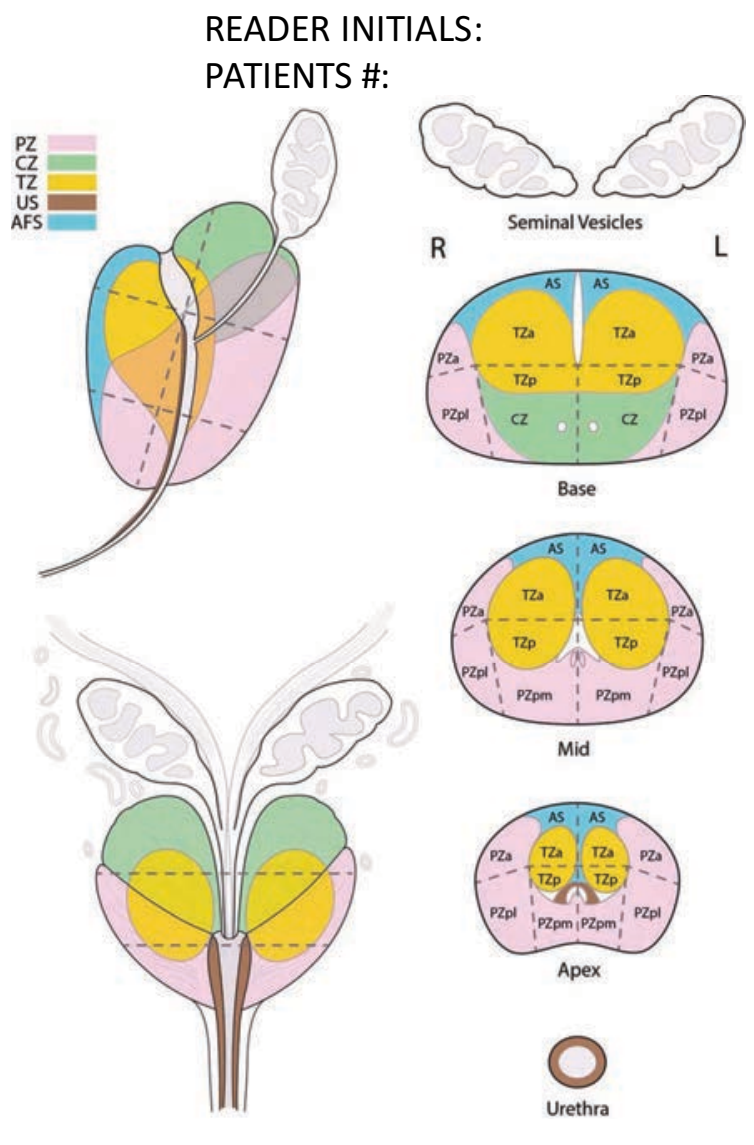

Figure 16 - Fiche d'évaluation lésionnelle prostatique

Analyse quantitative. L'étude était complétée par la mesure de l'intensité du signal au niveau d'une tumeur par comparaison au tissu sain prostatique. Un relecteur plaçait en référence des ROls au sein d'une zone de tissus d'apparence saine de la zone 
périphérique (ZP) et de la zone de transition (ZT) sur les séquences de diffusion (DWI) de valeur $b$ faible sur deux coupes différentes et les projetaient sur les niveaux de coupes correspondants en cartographie ADC. Secondairement, des ROls étaient placées sur la tumeur détectées la plus volumineuse. Il était ainsi mesuré le score ADC lésionnel minimum, moyen et la déviation standard.

\section{g. Analyses histologiques}

Après imagerie nocturne, la prostate était soumise au département d'anatomopathologie et placée dans une solution de formol à $10 \%$ pendant un jour. Après quoi, les surfaces externes étaient encrées comme présenté en Figure 17 avec les références couleurs suivantes $:$ rouge $=$ antérieur droit, vert $=$ droit postérieur, bleu $=$ antérieur gauche, noir = gauche postérieur. Le sommet était réséqué dans un plan perpendiculaire (antérieur à postérieur) et soumis pour analyse histologique en bloc de droite à gauche. Le bord du col de la vessie était aussi sectionné perpendiculairement (antérieur à postérieur) et soumis en bloc de droite à gauche. Des sections représentatives étaient enfin soumises (environ 98\% du spécimen) séquentiellement d'apical à basal et placées dans des cassettes ajustées par quadrant. Les tranches de section était ancrée au violet pour permettre l'orientation. La relecture était ensuite réalisée de façon conventionnelle par un uro-pathologiste dédiée.

\section{h. Analyses morphométriques de comparaison anatomo-clinique}

Au cours de la relecture histologique, les lésions cancéreuses étaient entourées manuellement sur les coupes en verre, avec leur score de Gleason respectifs. Dans le même temps, sur un document représentant une cartographie de coupes 
transversales de prostate, selon le nombre réalisé par pièce fraîche, les lésions étaient reproduites par l'anatomo-pathologiste suivant sa relecture de lames. Au maximum, on rapportait 3 lésions avec une numérotation de la plus importante (lésion index) à la plus petite. Les résultats de l'analyse de susceptibilité lésionnelle tumorale IRM étaient ensuite comparés par niveau de coupe correspondant selon une analyse visuelle. Cette comparaison ne prenait pas en compte les lésions histologiques de moins de 5cc, ni les envahissements des vésicules séminales. Cette analyse de concordance permettait de comparer chaque foyer lésionnel identifié à l'IRM aux résultats histologiques définitif. La concordance diagnostique était appréciée à plusieurs niveaux : présence tumorale dans la glande, par côté de la glande et par sextant. 

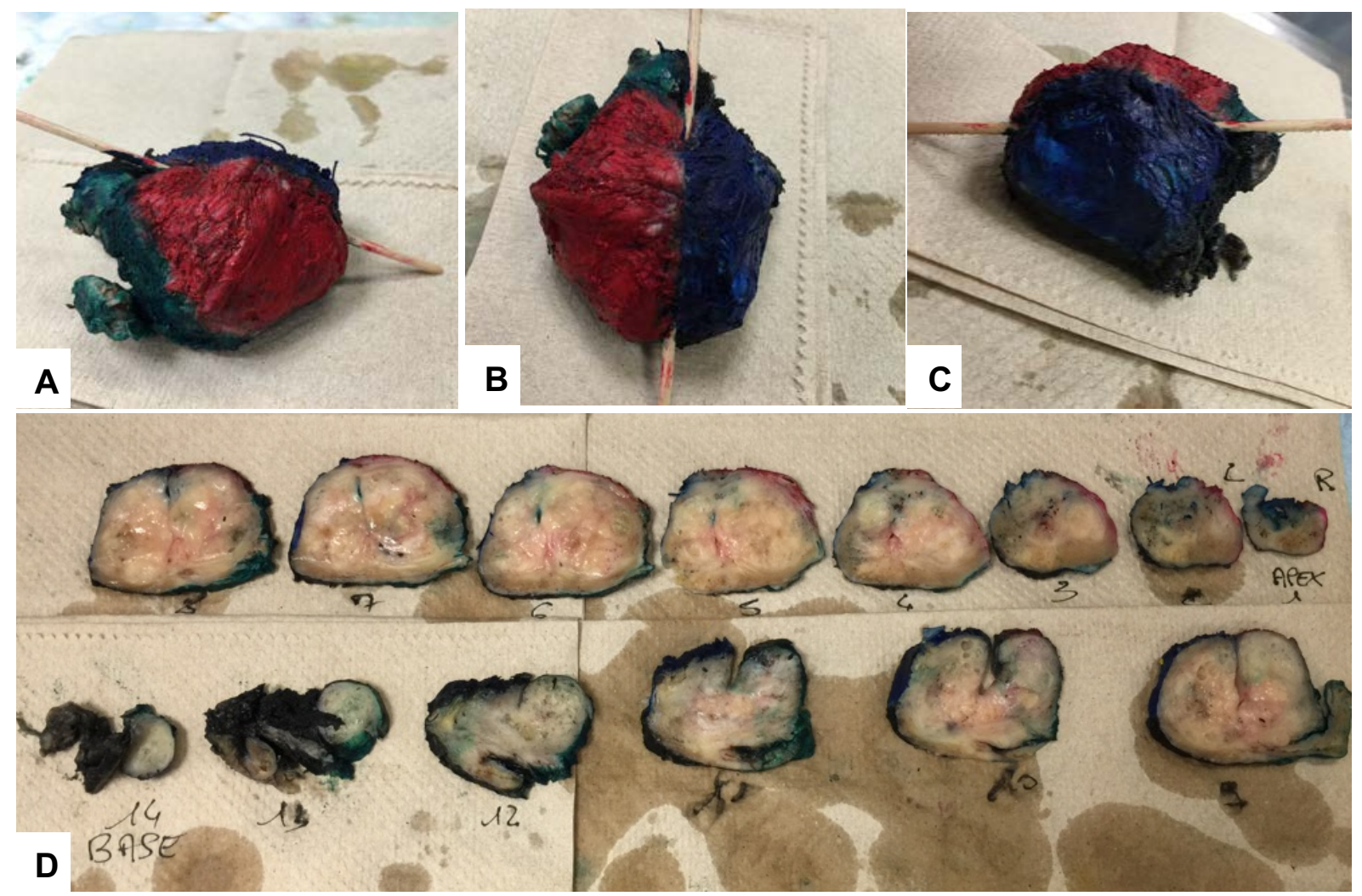

Figure 17 - Analyse histologique des échantillons de prostate

L'ancrage de la pièce avait lieu après $24 \mathrm{~h}$ passée dans le formol $10 \%$, à la suite de l'imagerie. La surface externe de l'organe était ancrée en rouge en antérieur droit $(A)$, en bleu en antérieur gauche $(C)$ depuis la partie la plus antérieure, jusqu'à la face postérieure qui était vert (postérieur droit) et noire (postérieure gauche). L'ensemble de la prostate était ensuite coupé dans un plan perpendiculaire à sa face postérieure de puis l'apex jusqu'à sa base et répartie par tranche numérotées $(D)$ pour préparer le travail de corrélation. Les pièces étaient ensuite coupées en demi ou en quadrant, en fonction de leur taille avec un ancrage violet sur les tranches de section. 


\section{i. Analyses statistiques}

Analyse de la qualité des images. La comparaison de l'interprétation de la qualité des images IRM 7T par les deux relecteurs indépendants en aveugle portait sur les 4 scores suivants : score global de qualité de la séquence en T2W, score global de la séquence en diffusion (DWI), score global de la cartographie ADC et score individuel d'évaluation qualitative morphologique correspondant à la somme des 3 scores précédents. Nous avons étudié la relation entre les interprétations des relecteurs grâce à l'utilisation d'indicateurs de corrélation et de concordance. La corrélation permettait d'étudier le lien entre deux distributions. La concordance, permettait d'étudier la capacité pour les deux relecteurs à fournir la même réponse. Pour chacun de ces deux axes d'étude, nous avons utilisé à la fois une approche graphique et numérique.

Les corrélations pour les différents critères étaient analysées grâce aux coefficients de corrélation de Spearman et aux droites de régression simple. La corrélation de Spearman permettait de mesurer la dépendance statistique non paramétrique entre deux variables.

La concordance était évaluée par le coefficient de corrélation intra-classe (ICC) et les graphiques de Bland-Altman [53]. L'ICC mesurait I'homogénéité des interprétations au sein des classes (ici les relecteurs) d'une variable continue par rapport à la dispersion de ces interprétations entre les classes. Les graphiques de Bland-Altman croisaient en ordonnée la différence d'interprétation des deux relecteurs (\#1 - \#2) avec en abscisse la moyenne de leurs interprétations (\#1 + \#2 / 2). 
Analyse des performances diagnostiques de I'IRM 7T. Les performances diagnostiques intrinsèques (sensibilité et spécificité) et extrinsèques (valeurs prédictives positives et négatives) de l'IRM étaient appréciées à la fois pour l'ensemble des lésions et pour les lésions indexes seulement. Chacune des imageries de prostate étaient analysées par secteur, selon 6 secteurs (base, médian, apex de droite et de gauche). Pour chacun d'entre eux, la suspicion lésionnelle IRM était comparée au gold standard de l'analyse histologique finale. En complément, la corrélation radiohistologique était appréciée par secteur et par ensemble de secteurs par le calcul du coefficient de corrélation kappa. 


\section{j. Résultats}

\section{Population \& échantillon d'étude}

Au global, 18 patients ont été sélectionnés consécutivement pour l'étude. Deux ont refusé de signer le consentement. Quatre ont été exclus pour des raisons techniques ou par manque de données. En particulier, un patient n'a pu bénéficier d'IRM 7T de sa pièce de prostatectomie radicale sur demande de l'opérateur qui souhaitait réaliser des biopsies extemporanées du spécimen pour vérifier l'absence de marge sur le lit de résection contre-indiquant l'imagerie IRM 7T. Les 3 autres cas ont été exclus a posteriori de I'IRM 7T pour perte d'acquisition des données IRM 7T, absence de séquence en 256 images ou artefact de mouvements. Pour ce dernier, il s'agissait d'une erreur de préparation du spécimen qui n'avait pas été assez fixé dans son container. Pour ce cas, l'analyse des images en diffusion (DWI) rendait compte de vibrations importantes de la pièce. Cela s'expliquait par le fait que la diffusion (DWI) qui utilise des gradients plus forts étaient alors responsables des microvibrations plus importantes à l'origine de la perte de netteté constatée. A noter que le dernier cas de la série (patient \#18) n'a pas eu de séquence de DWI ni de cartographie ADC mais une séquence $\mathrm{T} 2 \mathrm{~W}$ seule de qualité qui a été conservée pour analyse. La constitution de la cohorte d'étude est représentée dans le diagramme de flux en Figure 18. 


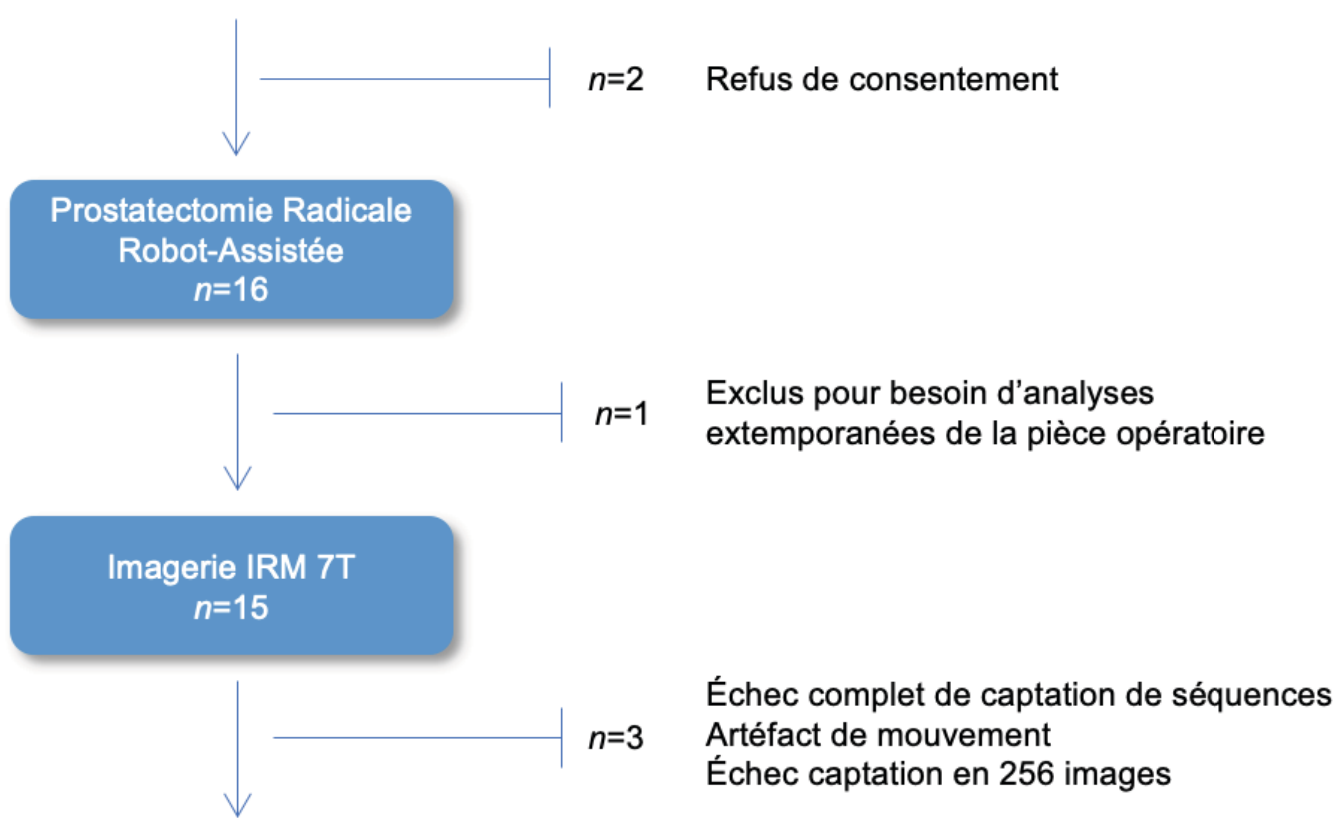

\section{Analyses}

morpho. \& lésionnelles $n=12$

Figure 18 - Diagramme de flux des patients de l'étude

Au total, l'étude portait sur 12 imageries biparamétriques IRM 7T post-opératoires de pièces de prostatectomies radicales de patients âgés de 64 ans en moyenne. Le descriptif précis des caractéristiques de la population d'étude est présenté dans le Tableau 7. Aucun des patients n'avait bénéficié d'un traitement antérieur de son cancer de prostate. Tous rapportaient une faible gêne urinaire (score IPSS moyen : 10/35) sans impact sur leur qualité de vie (score iQoL moyen : 2/6). Leur fonction sexuelle évaluée sur les 4 dernières semaines était globalement satisfaisante sur les 5 domaines analysés (score IIEF 15 moyen : 42/75). L'ensemble des patients a pu bénéficier d'une chirurgie d'exérèse prostatique complète robot-assistée avec 
technique de préservation neuro-vasculaire de grade 1,2 et 3 dans $38 \%, 38 \%$ et $23 \%$, respectivement [54].

Sur le plan oncologique, il s'agissait majoritairement de patients à risque intermédiaire de D'Amico $(n=9,75 \%)$. Dix d'entre eux avaient une tumeur T1c non palpable à l'examen clinique, découverte sur élévation de PSA. Le taux de PSA médian était de la cohorte était de $12 \mathrm{ng} / \mathrm{ml}(\min 2,3-\max 286)$. Aucune maladie ganglionnaire, $\mathrm{ni}$ métastatique n'était présente dans l'échantillon. En moyenne, 4 biopsies par patient étaient positives avec un $60 \%$ d'envahissement moyen par carotte. Les lésions étaient de score de Gleason $6(n=3,25 \%), 7(n=7,58 \%)$, et $>7 \quad(n=2,17 \%)$. Les tumeurs étaient localisées le plus fréquemment à l'apex (droit $67 \%$, gauche $50 \%$ ) et en base gauche $(50 \%)$.

A l'IRM préopératoire, 10 patients présentaient au moins une lésion significativement suspecte décrite comme telle sur l'imagerie clinique. Chaque anomalie de signal a pu être localisée et évaluée selon le score standardisé PI-RADS sauf une non scorée. On rapportait pour les lésions index de ces patient un score PI-RADS 3, 4 et 5 dans 1 (10\%), $4(40 \%)$ et $4(40 \%)$ cas, respectivement. Sur au moins 2 imageries de patients, une effraction capsulaire (T3a) était déterminée et 1 cas était suspect d'envahissement des vésicules séminales (T3b). Le volume prostatique moyen mesuré à l'imagerie était relativement modéré, autour de $30 \mathrm{ml}$, entre 14 et $46 \mathrm{ml}$. 
Tableau 7 - Caractéristiques cliniques \& histologiques préopératoires des patients de l'étude

Caractéristiques préopératoires

Variables

Nombre de patient, $n$

Âge moyen, années

64

IMC moyen, $\mathrm{kg} / \mathrm{m} 2$

28

Antécédent de traitement de cancer de prostate, $n$

0

PSA initial, $\mathrm{ng} / \mathrm{ml}$

Moyenne

12

Médiane

64,2

Min - Max

12,0

Stade cT, $n(\%)$

cT1c

$2,3-286,0$

cT2a

$10(84)$

cT2b

$0(0)$

cT2c

$1(8)$

$1(8)$

Stade $\mathrm{cN}, n(\%)$

cNO

12 (100)

$\mathrm{cN} 1$

$0(0)$

Stade M, $n(\%)$

MO

M1

$0(0)$

Score de Gleason, $n(\%)$

$<7$

$=7$

$>7$

2 (17)

1er grade de Gleason, $n(\%)$

9 (75)

3

3 (25)

4

$0(0)$

4 (34)

Biopsies positives par patient, moyenne (\%)

Pourcentage d'envahissement moyen d'une carotte, \%

urcentage d'envahissement max, \%

$7(58)$

Localisation des biopsies positives, $n(\%)$

Base droite

Base gauche

Médian droit

Médian gauche

$4(33)$

Apex droite

8 (67)

Apex gauche

$6(50)$

Classification de D'Amico, $n$ (\%)

Risque faible

Risque intermédiaire

Risque élevé

2 (17)

IRM préopératoires positives, $n$ (\%)

$10(84)$

Score PI-RADS, n (\%)

PI-RADS 3

PI-RADS 4

$4(40)$

PI-RADS 5

$4(40)$

Extension extra capsulaire à l'IRM, $n(\%)$

2 (17)

Envahissement des vésicules séminales à l'IRM, $n$ (\%)

Volume prostatique moyen à I'IRM, ml

30

IPSS, moyenne

10

iQol, moyenne

2

IIEF15, moyenne

42 


\section{Analyses histologiques finales \& suivi biologique des patients}

L'analyse a porté sur 12 échantillons de prostate d'un volume final médian de $42 \mathrm{ml}$, allant de 24 à $70 \mathrm{ml}$. La majorité des échantillons présentaient un remaniement adénomateux important dans $77 \%$ des cas associé à des lésion inflammatoires aigues dans $46 \%$ des cas. Les analyses rapportent aussi la présence de PIN de haut grade dans $46 \%$ des cas.

Au niveau tumoral, la taille moyenne des lésions index était mesurée à $1,3 \mathrm{~cm}$ de diamètre. En moyenne, $17 \%$ des glandes prostatiques étaient envahies par des lésions d'adénocarcinome prostatiques. Neuf cancers (75\%) étaient classés pT2, 1 (8\%) pT3a avec un net franchissement capsulaire et 2 (17\%) pT3b avec envahissement des vésicules séminales. Une $(8 \%)$ de ces deux lésions pT3b, présentait une marge positive (R1). Le reste de la population d'étude ( $n=11,92 \%)$, avait bénéficié d'une exérèse complète en marge saine, RO. Pour tous les patients, le stade histologique final est plus élevé que le stade préopératoire. Par ailleurs, aucun des curages réalisés n'a rapporté de ganglion envahi $(n=12,100 \%$ de $\mathrm{pN} 0)$. Tous les résultats postopératoires sont décrits dans le Tableau 8.

Sur le plan de l'analyse histopronostique, la majorité des tumeurs étaient évaluées avec un score de Gleason 7 dans $75 \%(n=9)$ des cas. Dans $50 \%$ des cas $(n=6)$, le score de Gleason final était identique à celui de l'analyse des échantillons sur les biopsies initiales. Dans 2 cas (17\%), on rapportait un score de Gleason plus élevé (upgrading de 17\%) et dans 4 cas, un score plus faible (downgrading de $33 \%$ ). 
Les taux de PSA à 1 mois post-opératoires étaient tous inférieurs à $0,02 \mathrm{ng} / \mathrm{ml}$ sauf 1 cas à $0,96 \mathrm{ng} / \mathrm{ml}$. Le succès chirurgical initial était donc estimé à $92 \%$. À 3 mois, puis à 12 mois, pour l'ensemble des patients, le taux de PSA était inférieur à 0,02 ng/ml. Aucun traitement complémentaire n'a été initié. Le taux d'échec thérapeutique a 1 an était donc de 0 .

Tableau 8 - Analyses histologiques et suivi biologique postopératoires des patients

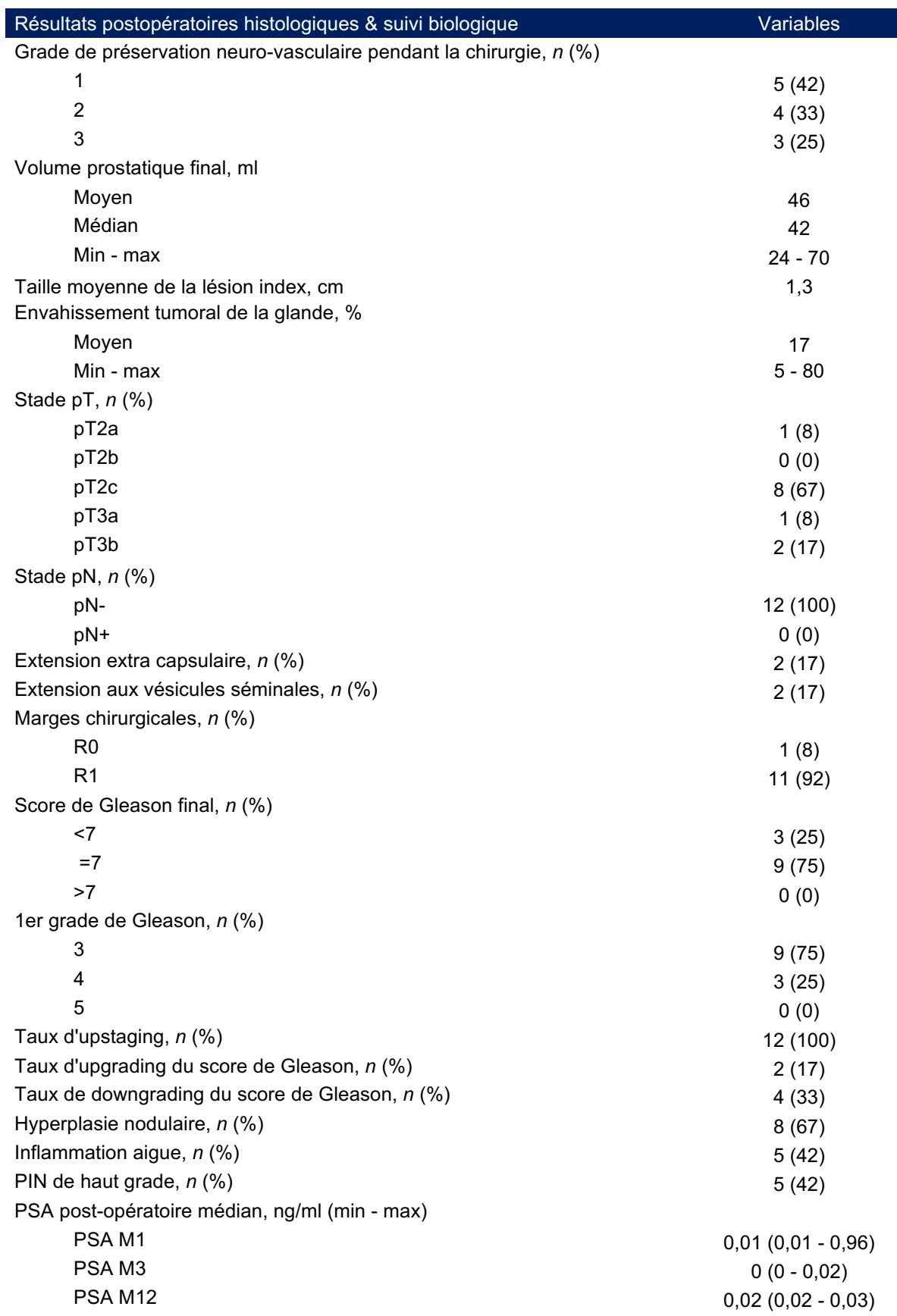




\section{Analyse morphologique qualitative de I'IRM 7T et concordance inter-relecteur}

L'analyse morphologique qualitative par scoring multicritère réalisée par les 2 relecteurs indépendants en aveugle, a porté sur 11 des 12 échantillons de la cohorte. L'absence de la séquence biparamétrique complète du dernier patient (\#18) empêchait l'évaluation chiffrée des séquences de diffusion (DWI) et de cartographie ADC pour ce patient. Au global, l'évaluation de la qualité des images IRM 7T par chacun des deux relecteurs étaient très satisfaisante pour les séquences en T2W. Le score global moyen de 12/15 était équivalent pour les 2 relecteurs. L'imagerie T2W en 256 images leur permettait à chacun d'identifier avec un haut niveau de discrimination tous les critères micro-anatomiques morphologiques recherchés. Le T2W était évalué de très haute qualité avec une résolution spatiale elle-même de grande qualité mais une résolution en contraste moins bonne. Le score moyen pour chacun des 3 critères d'analyse de qualité de la séquence T2W était de 4/5. Les séquences anatomiques T2W offraient aux relecteurs la possibilité de bien distinguer les pourtours de la glande, l'ensemble de l'anatomie zonale de McNeal, de suivre les contours de l'urètre, ceux des vésicules séminales, des canaux éjaculateurs et de délimiter précisément les bords des nodules d'hyperplasie prostatique.

Globalement la qualité des images en diffusion (DWI) était jugée moins bonne par les 2 relecteurs que celle de l'imagerie en T2W. De surcroît la séquence de diffusion ne couvrait pas toute la prostate. Le gain de résolution spatial ne permettait pas d'obtenir une imagerie de diffusion satisfaisante. Le score moyen de qualité de diffusion pour les 2 relecteurs est de 8,5/15. Les 3 critères d'analyse de la diffusion étaient affectés. Les artefacts étaient qualifiés comme fréquents par les 2 relecteurs et source de perturbations pour l'interprétation de signal. Les scores d'évaluation témoignaient 
aussi d'un phénomène de distorsion important. La netteté des images était jugée médiocre à modérée. L'ensemble dégradait la valeur du score global final de la séquence de diffusion (DWI). L'évaluation de la qualité de la cartographie ADC était en revanche bonne avec un score global moyen de qualité pour l'ADC de 4/5, équivalent pour les 2 relecteurs.

La concordance d'interprétation de lecture entre les 2 relecteurs complétait l'analyse. Seule l'évaluation de la qualité de l'imagerie en T2W présentait une corrélation significative. Le coefficient rho de Spearman était de 0,75 ( $p=0,004)$ (Tableau 9).

Tableau 9 - Corrélation de Spearman pour mesurer la dépendance statistique non paramétrique entre les évaluations des 2 relecteurs en fonction des séquences IRM 7T

$\begin{array}{lcc}\text { Séquences } & \text { Rho Spearman } & p \text { value } \\ \text { T2W } & 0,755 & 0,004 \\ \text { DWI } & 0,330 & 0,321 \\ \text { ADC } & 0,388 & 0,238 \\ \text { OVERALL } & 0,396 & 0,228\end{array}$

La régression linéaire était proche de la diagonale pour le scoring de cette seule séquence en T2W comme le montre la Figure 19. II n'y avait pas de corrélation entre les réponses des 2 relecteurs pour les scores de toutes les autres séquences: diffusion (DWI), cartographie ADC et score de qualité global (OVERALL) qui intégrait le score de chacune des séquences. Les régressions linéaires présentées en Figure 18 pour DWI, ADC et OVERALL montraient toutes la même tendance sans corrélation. L'analyse du comportement de lecture révélait que le relecteur \#1 avait tendance à sous-estimer, par rapport au relecteur \#2, les valeurs des résultats de faibles valeurs en leur attribuant un score plus bas. Les interprétations entre les relecteurs se rejoignaient pour les scores plus élevés. Ce résultat témoignait de l'existence d'une 
relation entre les distributions sans, pour autant, conclure à une proportion d'accord pour tous les niveaux de valeur.

La valeur du coefficient de corrélation intra-classe (ICC) était significative uniquement pour les scores de la séquence en T2W avec un taux de 0,61 ( $p=0,008)$. L'ensemble des autres coefficients intra-classe n'était pas significatif comme rapporté dans le Tableau 10. Ce résultat témoignait de l'existence d'un haut degré de concordance pour l'analyse des séquences en T2W.

Tableau 10 - Analyse de la concordance de relectures par calcul du coefficient de corrélation intra-classe appliqué pour l'interprétation de chacune des séquences IRM 7T

$\begin{array}{lcc}\text { Séquences } & \text { ICC } & p \text { value } \\ \text { T2W } & 0,61 & 0,008 \\ \text { DWI } & 0,35 & 0,130 \\ \text { ADC } & 0,37 & 0,097 \\ \text { OVERALL } & 0,33 & 0,130\end{array}$

En complément, la représentation graphique de Bland-Altman, en Figure 20 présentait une excellente concordance globale entre les deux évaluations des relecteurs. A noter cependant que les valeurs présentes en dehors des limites de l'intervalle de confiance correspondaient toutes à des scores faibles, confirmant les résultats présentés précédemment sur les courbes de régression linéaire. 


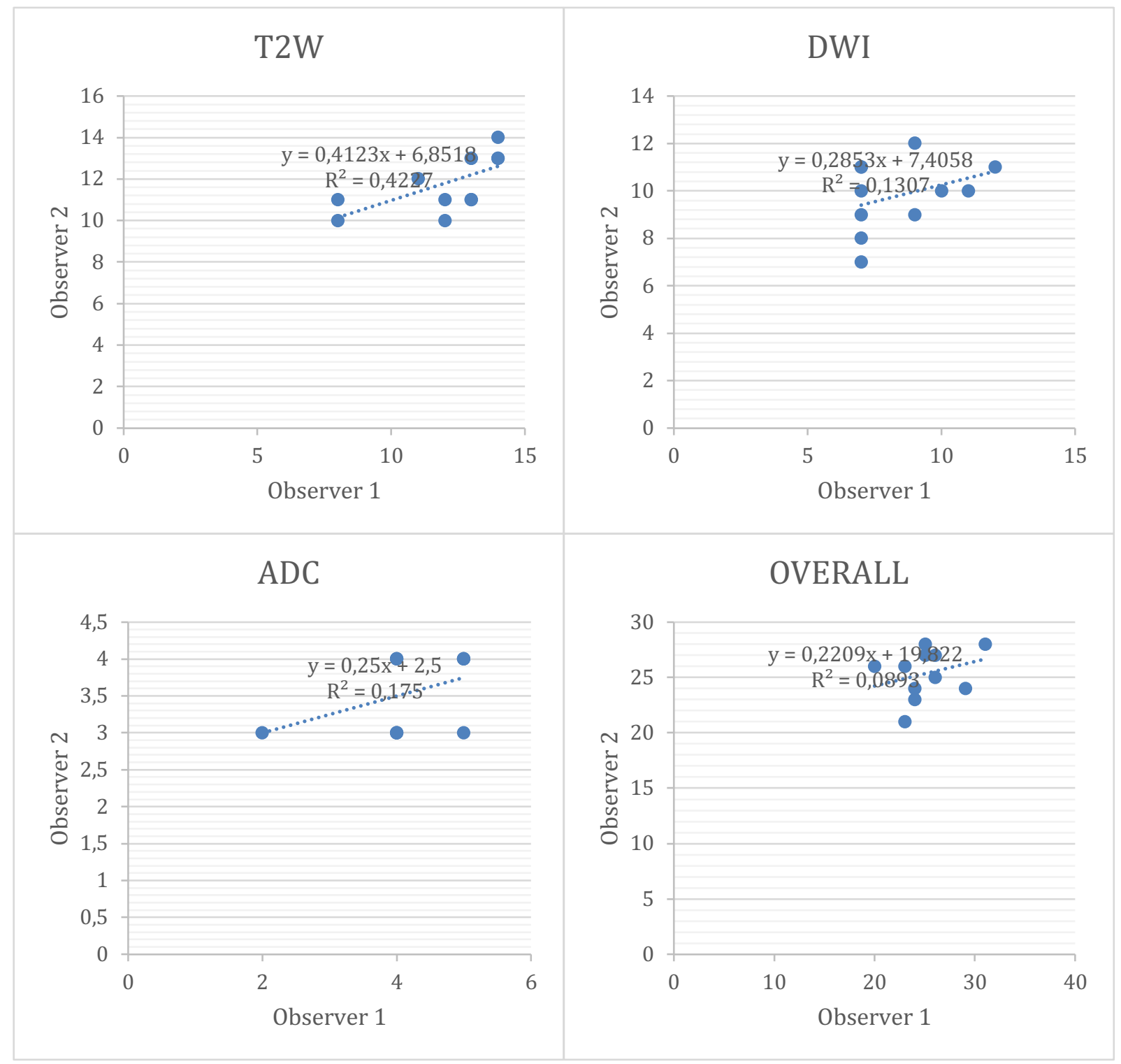

Figure 19 - Corrélation des interprétations de la qualité des imageries IRM 7T par régression linéaire

L'analyse portait sur la comparaison des données qualitatives de l'interprétation des 4 scores suivants : score global T2W, score global DWI, score ADC et score individuel d'évaluation qualitative morphologique. La régression linéaire en $\mathrm{T} 2 \mathrm{~W}$ est proche de la diagonale. 
T2W

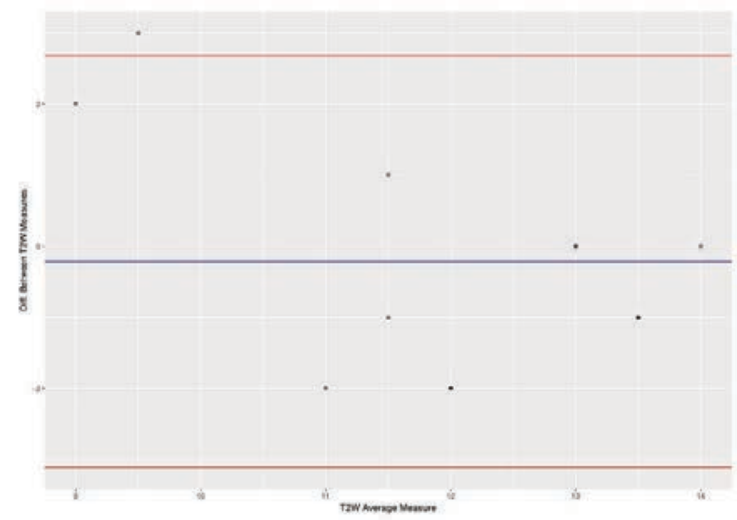

$\mathrm{ADC}$

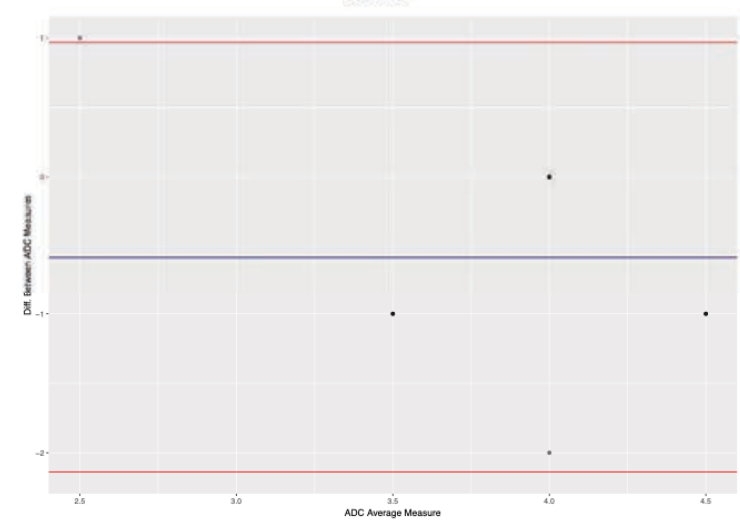

DWI

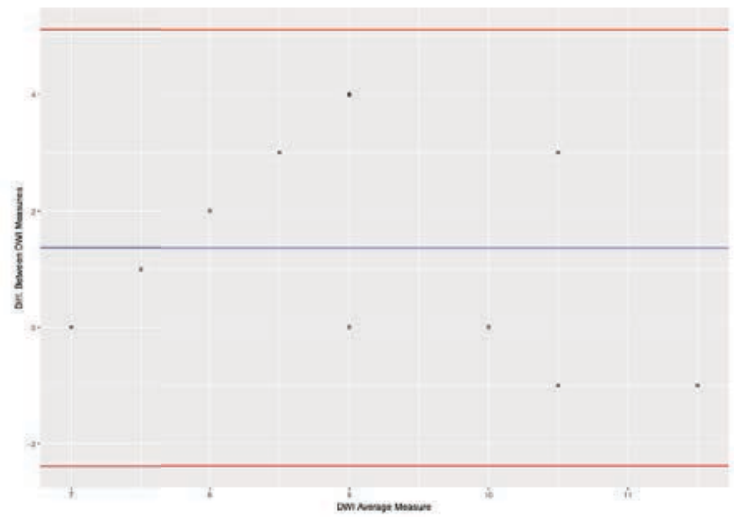

OVERALL

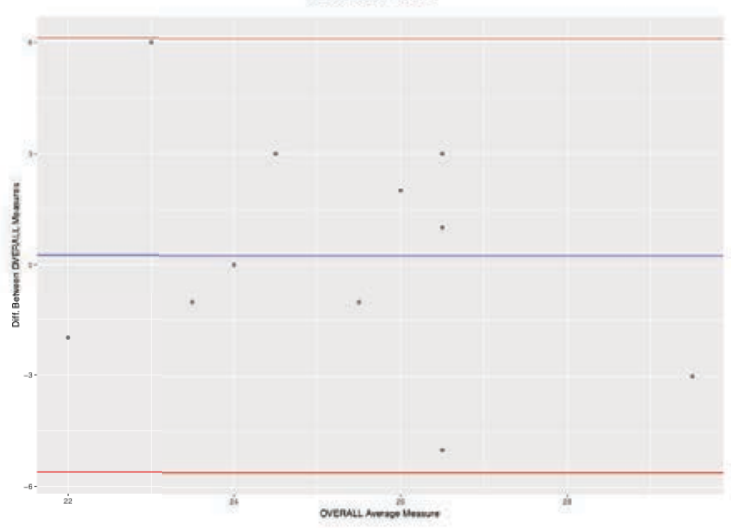

Figure 20 - Concordance des interprétations de qualité des imageries IRM 7T selon Bland-Altman

On remarque une excellente concordance globale entre les relecteurs. Les réponses en dehors des limites de l'intervalle de confiance s'appliquent toutes pour des scores de faibles valeurs pour lesquelles il y a peu de concordance. 
Au global, la qualité de l'imagerie biparamétrique obtenue permettait aux relecteurs de suivre le schéma de lecture systématique recommandé en IRM. II était possible de passer en revue de façon indépendante les 3 principaux compartiments de la prostate, à savoir : la zone périphérique (ZP), la zone de transition (ZT) et le SFMA. Ces zones étaient toutes pleinement identifiables en IRM 7T sur chacune des séquences, et plus particulièrement en T2W. La Figure 21 rend compte de l'adaptation du schéma de partitionnement de la prostate, issu de la conférence de consensus PREDICT [55], représenté ici sur des coupes de séquence anatomique en T2W. On note que les repères radio-anatomiques usuellement utilisés étaient tous identifiables en IRM 7T. Cette cartographie en 27 secteurs permettant de localiser précisément et de façon conventionnelle toutes les lésions suspectées était applicable à ce modèle d'imagerie haute résolution. Le haut niveau de signal obtenu avec l'IRM 7T permettait d'assurer une orientation dans les différents plans selon les recommandations habituelles. Le recours au référentiel commun en 27 secteurs pour la localisation des lésions et la description plus globalement de la glande prostatique était réalisable sans aucun aménagement des conventions existantes définies en IRM clinique actuelle. 

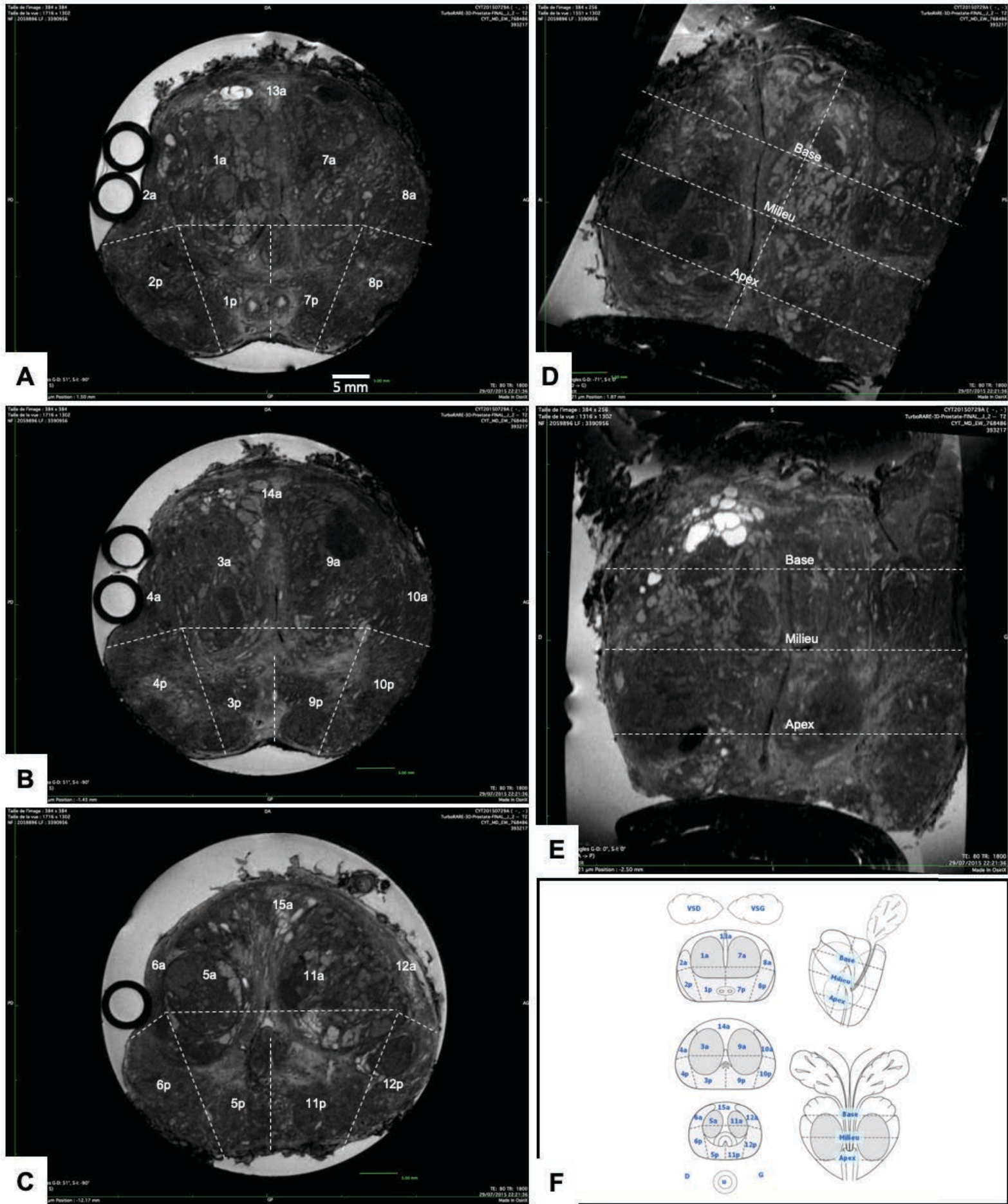

Figure 21 - Coupes IRM 7T cartographiées selon le schéma de partitionnement de la prostate recommandé par la conférence de consensus PREDICT (Londres, 2010) [55] 
Sur la Figure 22, on peut suivre avec précision le trajet de l'urètre prostatique. II naît de la base de la glande au niveau du col vésicale, au sein de la zone antérieure du SFMA, en zone 13a. Le SFMA apparaît sur cette imagerie en hyposignal modéré. A noter que la série d'image ne fait pas apparaître constamment cette zone antérieure de façon clairement identifiable. On distingue la coupe urétrale identifiable à sa forme triangulaire à base postérieure. Il est en franc hyposignal T2W sur l'imagerie quand sa lumière est occluse. II prend une direction oblique au centre de la zone de transition, en bas et en avant. A la portion moyenne de la glande sa base repose sur le bord supérieur de des deux zones postéro-médianes périphériques selon un trajet presque horizontal jusqu'à l'apex prostatique très reconnaissable dans le plan sagittal. Le veru montanum apparaît dans le plan sagittal à la l'union des deux-tiers proximaux et du tiers distal de l'urètre, légèrement plus en hypersignal en décrivant une forme nodulaire. La couche musculaire lisse qui constitue le plan profond sous-muqueux de l'urètre est décrite comme une plage harmonieuse en hypersignal homogène qui circonscrit l'ensemble de la filière urétrale prostatique longitudinalement, bien identifiable dans le plan sagittal (disque blanc en Figure 22 (D)). 


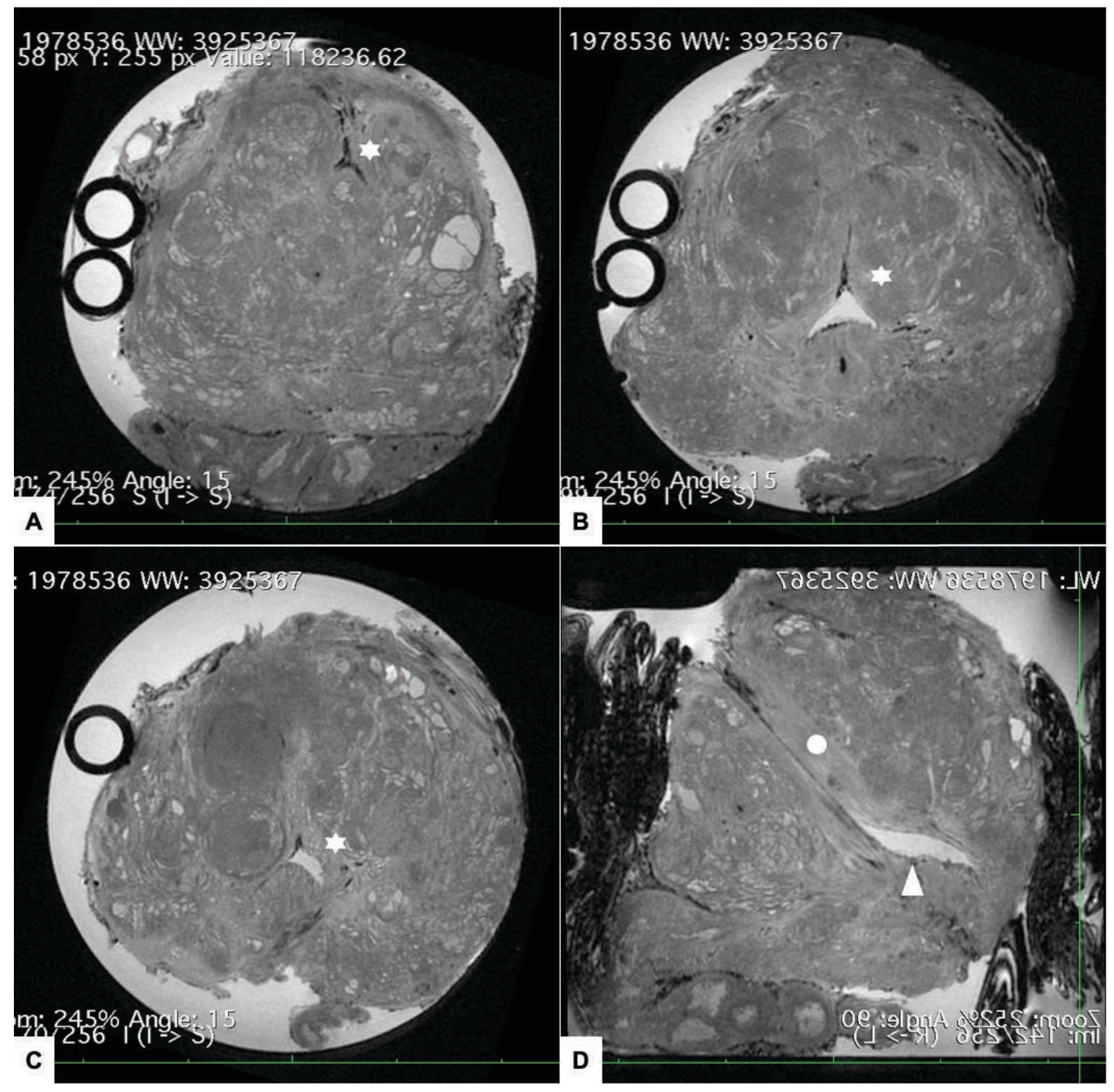

Figure 22 - Coupes IRM 7T présentant le trajet de l'urètre

II s'agit d'une série d'images de pièce de prostatectomie radicale, TR $1500 \mathrm{~ms}$, TE $80 \mathrm{~ms}$, de champ visuel de $5 \times 5$ $\mathrm{cm}$ avec une épaisseur 0,195, réalisé en $4 \mathrm{~h} 25 \mathrm{~min}$ à une résolution spatiale de $195 \times 195 \times 195 \mu \mathrm{m} 3$. L'urètre (étoile) apparaît sur les coupes frontales de la base $(A)$ à la portion moyenne de la glande (B) jusqu'à l'apex (C) avec une lumière en hypersignal, un fin liséré crénelé en hyposignal profond suivant les contours nets de la muqueuse urétrale. En coupe sagittale, il suit un trajet oblique en avant depuis son origine jusqu'au veru montanum (triangle blanc) où il adopte une direction plane horizontale jusqu'à l'apex prostatique. Dans toute sa longueur, il est circonscrit de plage en hypersignal longilignes (disque blanc) correspondant à sa musculature lisse sousmuqueuse. 
La Figure 23 représente une image d'IRM 7T en séquenceT2W, avec les paramètres suivant TR 1500, TE 70, CV $5 \mathrm{~cm}$, pour une capture en 256 images permettant d'obtenir une résolution de $195 \times 195 \times 195$ en 6 h50 de temps d'acquisition. Cette série d'imagerie était noté $4 / 5$ par les 2 relecteurs concernant le critère d'analyse d'identification de l'anatomie zonale de McNeal et des limites de l'urètre, avec un score global multicritère en T2W de 13/15. II s'agissait d'une image ayant parmi les meilleurs scores d'évaluations des relecteurs. Elle offrait une visualisation très précise de l'architecture histologique urétrale ici représentée sur une coupe transversale entre la portion moyenne et apicale de la prostate. On distinguait très nettement les différentes couches histologiques constitutives de l'urètre qui se superposent à une échelle inframillimétrique. On rapportait une discordance axial-sagittal dans la reconnaissance de ces couches pariétales urétrales.

Sur le zoom réalisé dans la zone d'intérêt (carré en ligne pointillé blanc), on reconnaît la muqueuse, en hyposignal franc T2, avec son urothélium (U) sans distinction possible de la lamina propria. On peut suivre très nettement le plissement de l'urothélium en l'absence de distension de ses parois par manque d'urine dans la lumière en hypersignal de l'urètre. Les couches musculeuses internes, moyennes, externes et l'adventice de la sous-muqueuse sont toutes confondues (étoile blanche). Elles apparaissent en hypersignal modéré contrastant nettement avec la muqueuse en hyposignal franc. Un signal plus hétérogène caractéristique du tissu prostatique de la zone de jonction et de la zone périphérique marque la fin de cette structure. 


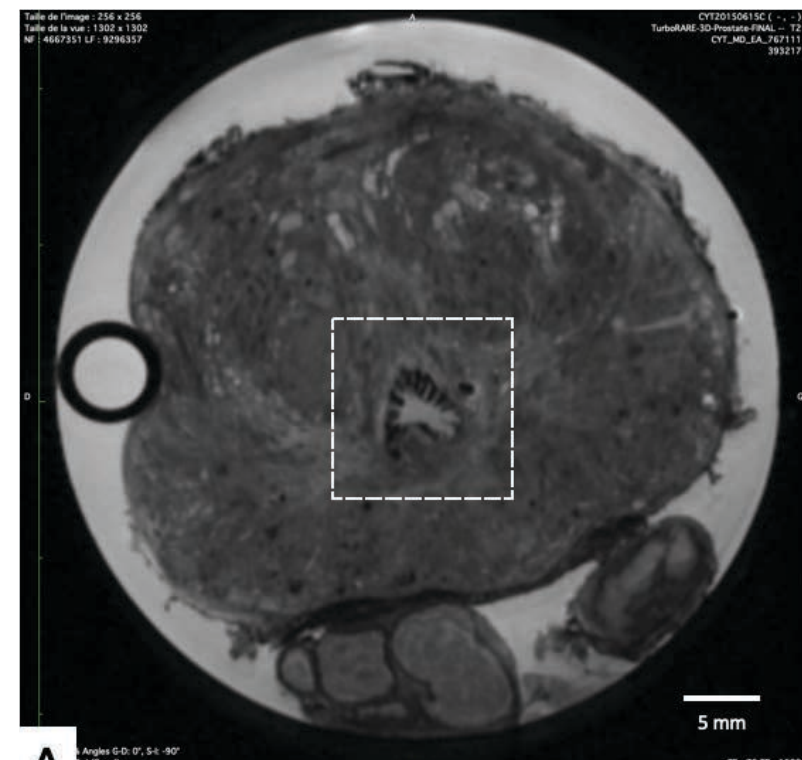

A

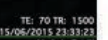

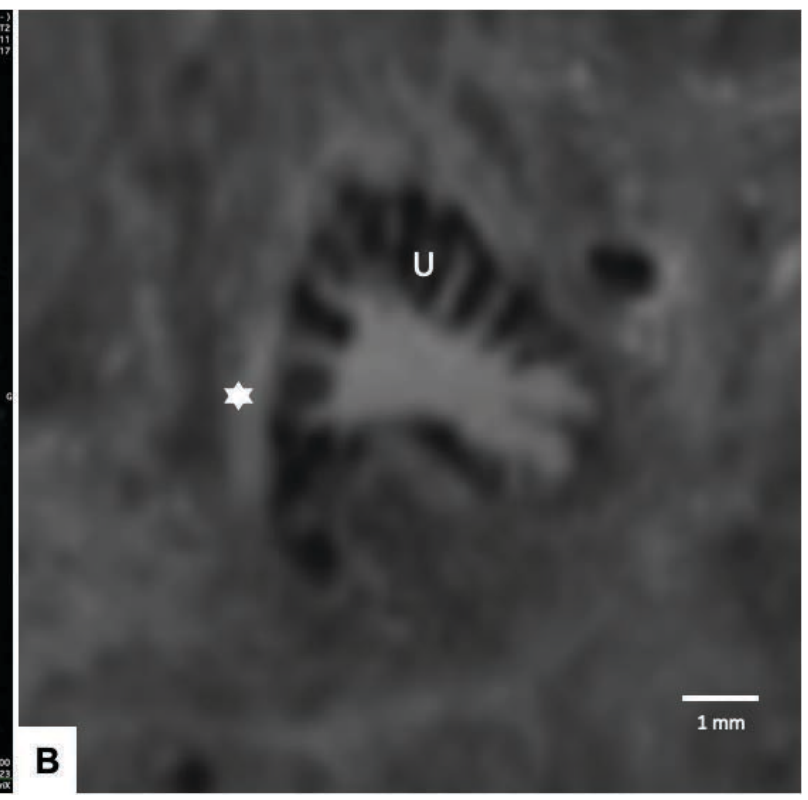

Figure 23 - Coupes IRM 7T de la micro-anatomie urétrale

II s'agit d'une image issue d'une séquence T2W de 256 images avec un TR 1500, TE 70, CV $5 \mathrm{~cm}$, offrant une résolution de $195 \times 195 \times 195$ en 6 h50 de temps. En (A), l'image passe en coupe transversale au niveau de la portion moyenne/apicale de la prostate. Le carré blanc en ligne pointillé encadre la région d'intérêt qui est zoomé en (B). L'urothélium est identifiable avec une précision infra-milimétrique. En hyposignal profond, on remarque le plissement de la muqueuse urothéliale $(U)$. Les sous-couches concentriques musculaires apparaissent en hypersignal (étoile) sur une épaisseur de $0,5 \mathrm{~mm}$.

Les canaux éjaculateurs apparaissaient très distinctement sur la totalité des imageries en T2W. Plusieurs coupes sont représentées en Figure 24. On pouvait suivre leur naissance depuis les vésicules séminales à la base prostatique. Les canaux éjaculateurs sont très reconnaissables, ils sont centrés par une lumière en hypersignal légèrement crénelé dans sa portion basale la plus charnue. Ils sont entourés d'un anneau de forme oblongue en hyposignal modéré T2. Ils évoluent côte à côte, de part et d'autre d'une ligne verticale qui passe en coupe transversale par le centre de l'urètre. Ils poursuivent leur trajet oblique en haut et en avant pour se jeter dans l'urètre prostatique, au niveau du veru montanum. 


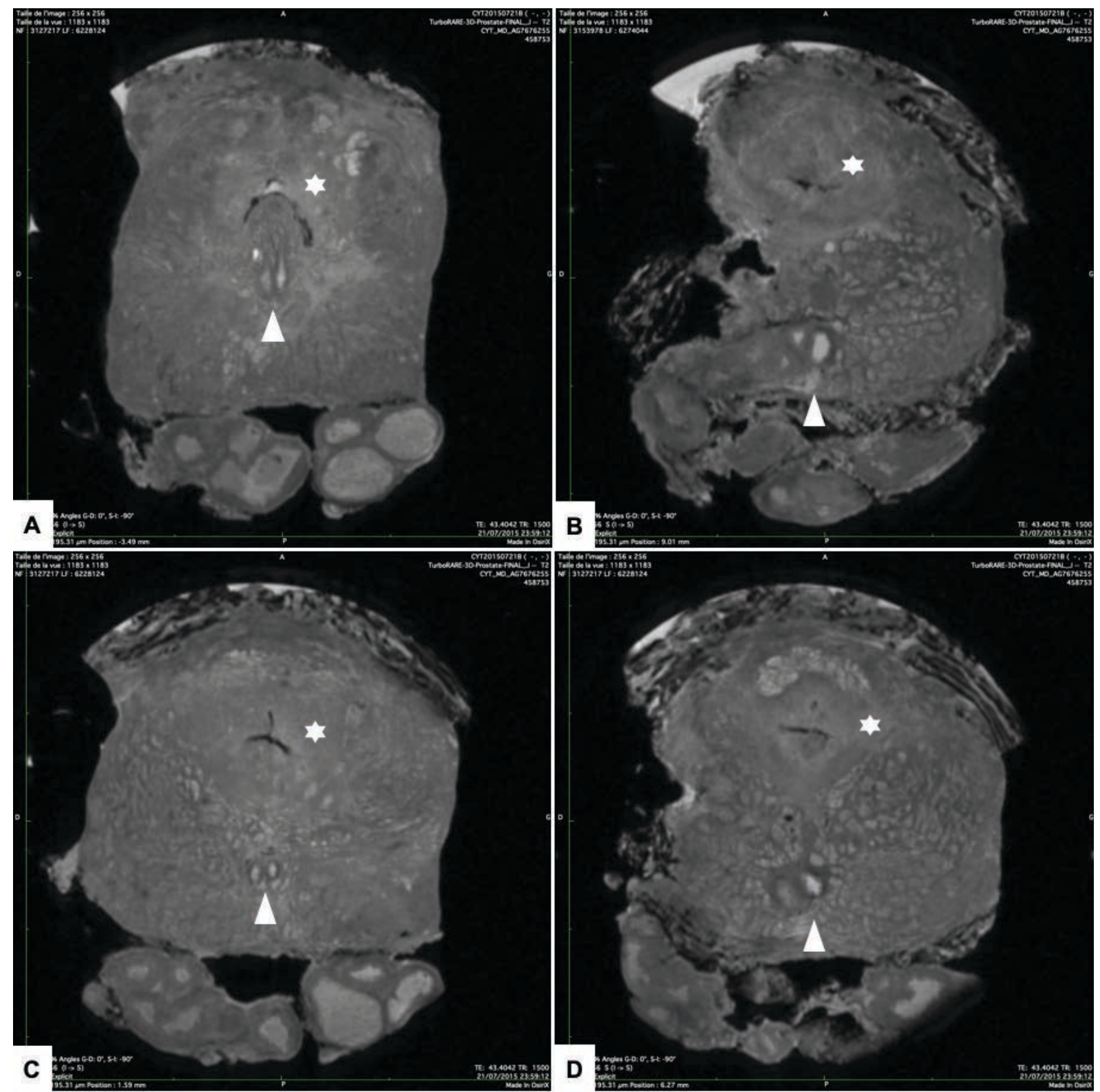

Figure 24 - Coupes IRM 7T présentant plusieurs coupes des canaux éjaculateurs

II s'agit d'une série d'images de pièce de prostatectomie radicale, TR $1500 \mathrm{~ms}$, TE 43 ms, de champ visuel de $5 \times 5$ $\mathrm{cm}$ avec une épaisseur 0,195, réalisé en $6 \mathrm{~h} 50 \mathrm{~min}$ à une résolution spatiale de $195 \times 195 \times 195 \mu \mathrm{m} 3$. On peut suivre le trajet des canaux éjaculateurs depuis leur naissance au niveau des vésicules séminales jusqu'à leur extrémité distal dans le canal de l'urètre (étoile blanche). Ils sont très facilement identifiables sur toutes les coupes. Ils sont centrés par un hypersignal intense et entouré d'un contour en hyposignal profond et homogène (pointe blanche). 


\section{Analyse de susceptibilité lésionnelle IRM, performance \& concordance radio-}

histologique

L'analyse de corrélation a porté sur les 12 cas représentés dans la Figure 25. Au total, 22 foyers suspects de cancers de prostate, de $10 \mathrm{~mm}$ en moyenne de grand axe, étaient rapportés sur 12 séries d'imageries IRM 7T de pièces de prostatectomies radicales fraîches dont 11 bénéficiaient d'imageries bi-paramétriques (T2W, DWI et ADC). On identifiait donc en moyenne 2 foyers suspects par prostate. Précisément, l'analyse comprenaient 12 lésions indexes, 7 lésions secondaires et 3 lésions tertiaires de $13 \mathrm{~mm}, 6 \mathrm{~mm}$ et $5 \mathrm{~mm}$, de grand axe respectivement. Le foyer le plus volumineux mesurait $25 \mathrm{~mm}$ et la plus petit $4 \mathrm{~mm}$. L'ensemble des caractéristiques des foyers suspects de malignité en IRM 7T a été analysé et présenté dans le Tableau 11.

Tableau 11 - Analyse des foyers suspects de lésions tumorales à I'IRM 7T

$\begin{array}{lcccc}\text { Lésions suspectes à l'IRM 7T } & \text { Toutes } & \text { Index } & \text { Secondaire } & \text { Tertiaire } \\ \text { Nombre de foyers suspects, } n & 22 & 12 & 7 & 3 \\ \text { Taille, cm } & 10 & 14 & 6 & 5 \\ \quad \text { Moyenne } & 7 & 14 & 6 & 5 \\ \quad \text { Médiane } & 4-25 & 7-25 & 5-8 & 4-5 \\ \quad \text { Min - max } & 20(91) & 11(92) & 6(86) & 3(100) \\ \text { Hypointense T2, } \mathrm{n}(\%) & 13(59) & 7(59) & 6(86) & 0(0) \\ \text { Hyperintense en DWI, } \mathrm{n}(\%) & 18(82) & 10(83) & 6(86) & 2(67) \\ \text { Restriction ADC, } \mathrm{n}(\%) & & & & 3 \\ \text { Score de perceptibilité lésionnelle, moyenne } & 3 & 3 & 2 & 1 \\ \quad \text { en T2W } & 2 & 2 & 2 & 1 \\ \quad \text { en DWI } & 3 & 3 & 3 & 2 \\ \quad \text { en ADC } & & & & 2 \\ \text { Score de perceptibilité lésionnelle global } & 3 & 3 & 2 & 1-2 \\ \quad \text { Moyenne } & 2 & 3 & 2 & 2-4 \\ \quad \text { Médiane } & 1-5 & 2-5 & & \end{array}$



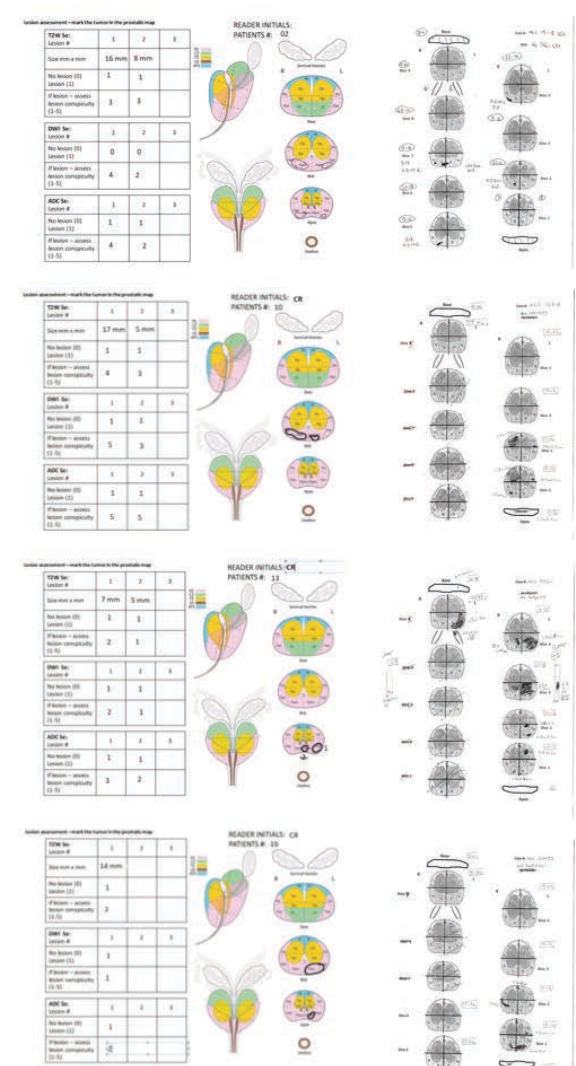
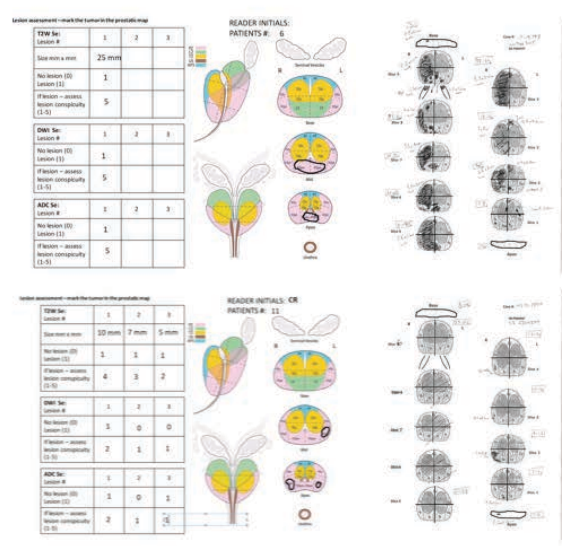

$-5=$
-40
-40
$-4=$

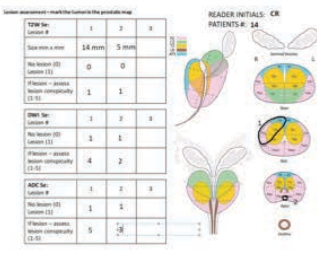

$5=$
-15
-95
$-9=$
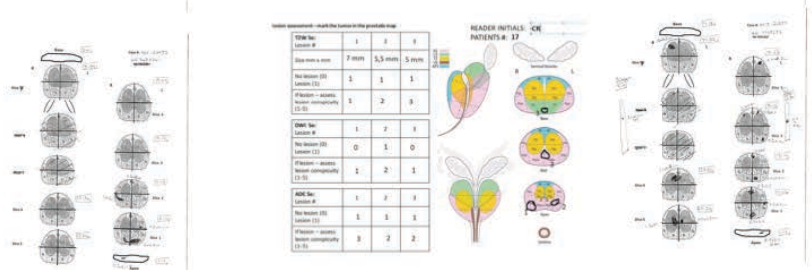
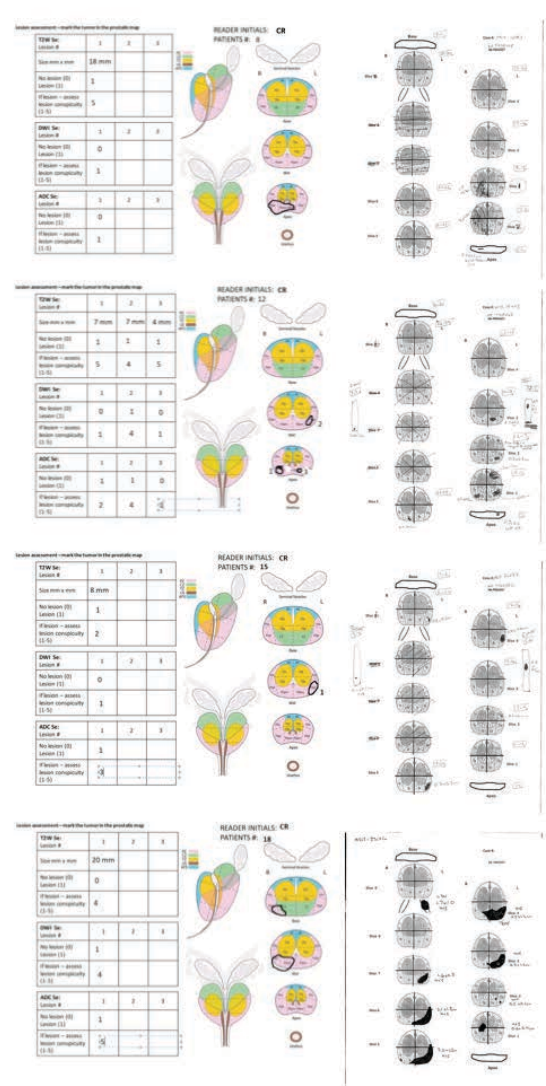

\section{Figure 25 - Schémas d'étude de corrélation radio-histologique à l'aveugle}

Le schéma de gauche était complété par l'uro-radiologue, en l'absence de toute information sur le patient. Le relecteur représentait sur le schéma des coupes prostatiques la localisation de la zone suspectée pour cancer de prostate. II devait caractériser en plus l'évaluation de la lésion selon les séquences IRM 7T. Le schéma de droite représente la cartographie complète réalisée par l'anatomopathologiste. II reportait sur un nombre de coupes de prostate proportionnelle à sa volume la localisation précise et les rapports anatomique de la lésion, en renseignant sur son grade. 
Globalement, on notait que majoritairement, dans $91 \%(n=20)$ des cas, les lésions décrite comme suspectes étaient hypo T2 avec dans $89 \%(n=18)$ une restriction de I'ADC par rapport au tissu environnant. L'intensité de signal en diffusion semblait moins affectée au niveau de ces foyers suspects où l'on ne rapportait un hypersignal en DWI que dans seulement $59 \%(n=13)$ des cas. Les foyers étaient classés comme moyennement visibles avec un score moyen et médian de perceptibilité lésionnel global de $3 / 5$ et $2 / 5$ respectivement. Seuls les foyers des lésions indexes les plus volumineuses en T2W étaient jugées comme bien visibles avec une médiane de score de perceptibilité de $4 / 5$.

Tous les foyers suspects de cancer de prostate à l'imagerie IRM 7T ne correspondaient pas à des lésions malignes confirmées par histologie finale. Les analyses définitives des échantillons de prostatectomies ont identifié 25 lésions d'adénocarcinomes prostatiques significatifs (>0,2 cc), soit 2 en moyenne par prostate. Les indicateurs de performance diagnostique de I'IRM 7T pour la détection et la localisation des tumeurs, toute lésion confondue, étaient les suivants : sensibilité (Se) : 70\%, spécificité (Sp): 80\%, valeur prédictive positive (VPP) : 79\% et valeur prédictive négative (VPN) : 72\%. Ces tests s'amélioraient dans l'ensemble, sauf pour la sensibilité, quand on considérait uniquement la détection et la localisation de la lésion index par prostate avec les résultats suivants : Se : $65 \%, \mathrm{Sp}: 92 \%$, VPP : 79\% et VPN : $85 \%$.

Les coefficients kappa révélaient des résultats assez disparates autant entre l'ensemble des lésions et la lésion indexe, qu'entre les différents quadrants. Les plus grandes corrélations rapportées pour l'ensemble des lésions étaient atteintes en base, 
notamment à droite et en médian droit. Pour les lésions indexes les meilleurs scores étaient atteints en médian droit et gauche, avec plus de réserve sur le médian global, et sur la prostate gauche en particulier l'apex gauche. L'ensemble des résultats de l'étude de corrélation par secteur et des tests de performances IRM 7T est présenté dans le Tableau 12.

Tableau 12 - Analyse de corrélation radio-histologiques \& performances diagnostiques IRM 7T

$\begin{array}{lcc} & \text { Toutes lésions } & \text { Lésions indexes } \\ \text { Nombre total de lésions histologiques finale, } n & 25 & 12 \\ \text { Moyenne par prostate } & 2 & n a \\ \text { Médiane par prostate } & 2 & n a \\ \text { Corrélation toutes lésions par secteur, kappa [IC95] } & & 0,4[-0,154 ; 0,954] \\ \text { Hémiprostate droite } & 0,429[-0,165 ; 1,000] & 0,833[0,525 ; 1,000] \\ \text { Hémiprostate gauche } & 0,226[-0,166 ; 0,618] & 0,429[-0,165 ; 1,000] \\ \text { Base } & 0,571[0,082 ; 1,000] & 0,471[-0,035 ; 0,976] \\ \text { Médian } & 0,389[-0,303 ; 1,000] & 0,471[-0,035 ; 0,976] \\ \text { Apex } & 0,429[-0,165 ; 1,000] & 0[n a] \\ \text { Base droite } & 0,625[0,027 ; 1,000] & -0,125[-0,303 ; 0,053] \\ \text { Base gauche } & 0,25[-0,372 ; 0,872] & 0,824[0,498 ; 1,000] \\ \text { Médian droit } & 0,833[0,525 ; 1,000] & 0,625[0,155 ; 1,000] \\ \text { Médian Gauche } & 0,273[-0,254 ; 0,799] & 0,333[-0,200 ; 0,867] \\ \text { Apex droit } & 0,143[-0,403 ; 0,688] & 0,625[0,155 ; 1,000] \\ \text { Apex gauche } & 0,471[-0,035 ; 0,976] & \\ \text { Performances diagnostiques de I'IRM } 7 \text { T, } \% & & 65\end{array}$

Le déficit de corrélation lors l'interprétation en aveugle, reposait dans certains cas, sur la considération d'anomalie de signal classés à tort comme non significatif par le relecteur. L'étude de corrélation du cas \#6 notamment, montrait que la lésion était très sous-évaluée à l'analyse de l'imagerie. Seulement 4 secteurs sur 6 étaient rapportés par le relecteur comme suspects d'envahissement tumoral. L'erreur était due à la présence de trop nombreuses anomalies de signal en hypo T2, avec hypersignal en diffusion et une forte restriction ADC qui faisaient conclure à des images construites compte-tenu de l'envahissement jugé trop important. Ce cas est présenté sur la Figure 
26 qui correspondait à l'imagerie d'une volumineuse lésion de score de Gleason 7 $(4+3)$. Ici sur la coupe en partie moyenne, la tumeur se développait au dépend de l'ensemble du lobe prostatique droit, de façon depuis le SFMA jusqu'à la ZC en épargnant partiellement la ZP droite. Cette zone présente des caractéristiques suspects en imagerie avec un hyposignal franc en T2W, un hypersignal en diffusion et une restriction $A D C$ intense. Toutefois la localisation jonctionnelle et antérieure moins fréquente pour les tumeurs, l'existence de nodules d'hyperplasie prostatique bilatéraux parasitant la lecture ont fait conclure à tort à un faux négatif sur la zone positive pour le cancer de prostate.

La corrélation pouvait être très élevée sur des analyses de cas comme le montre la Figure 27. Dans ce cas \#15, la zone de suspicion tumorale définie par le relecteur est superposable à la localisation cancéreuse validée par l'histologie finale. La concordance était parfaite pour une lésion de $8 \mathrm{~mm}$ postéro-latérale gauche en hypo signal T2 avec une restriction ADC modéré, sans altération sur la séquence en diffusion. C'est dans ce cas, principalement, l'analyse de la séquence anatomique en T2 avec la concordance de plusieurs critères morphologiques dans la zone postérolatérale (hypo T2 et plage de signal homogène par rapport au tissu sain adjacent) qui font caractériser par le relecteur la lésion comme suspecte, bien que le signal ne soit pas modifié en diffusion. 

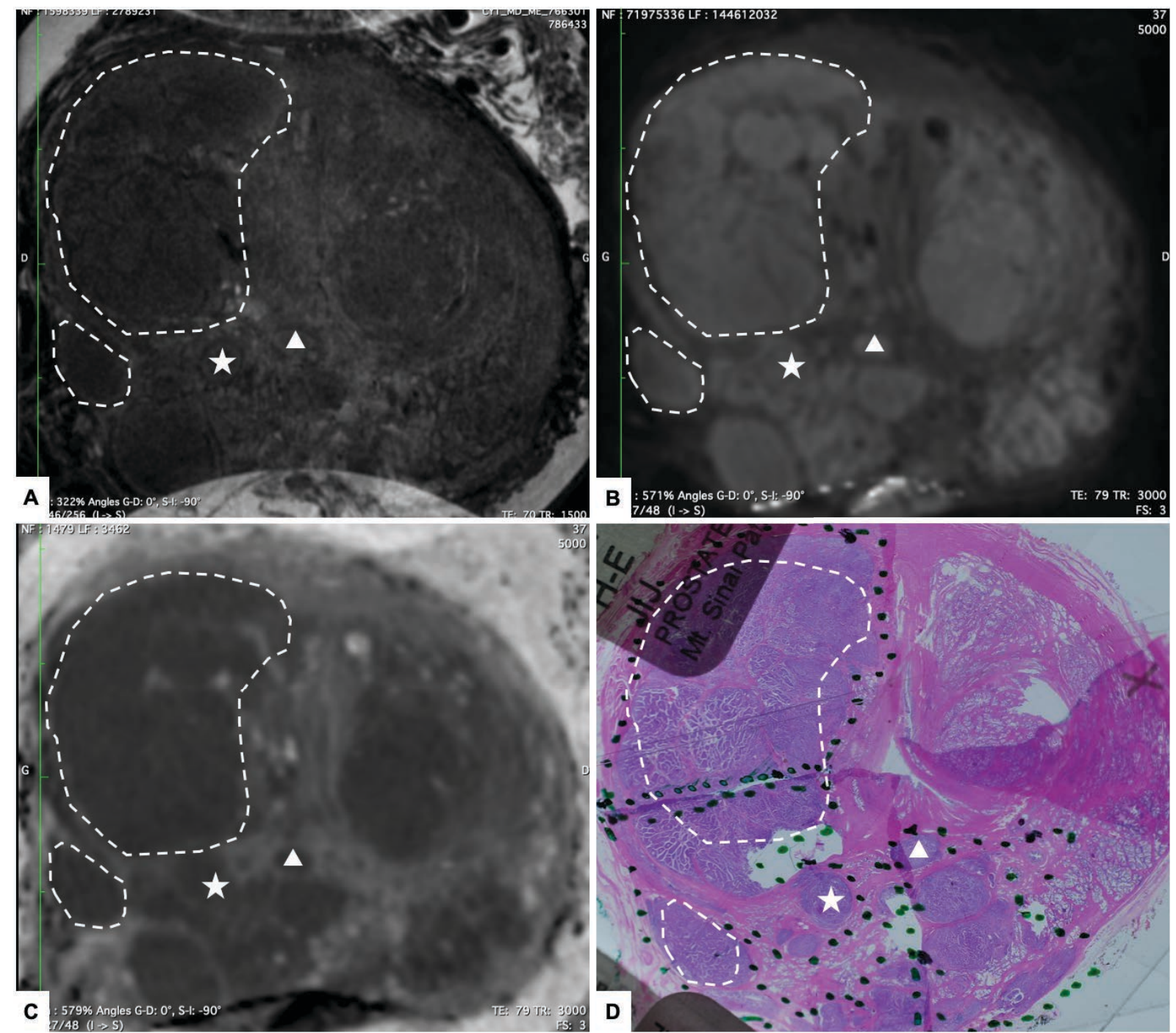

\section{Figure 26 - Imagerie IRM 7T biparamétrique d'une volumineuse lésion prostatique de score de Gleason 7}

Homme de 64 ans, obèse avec un IMC à $34 \mathrm{~kg} / \mathrm{m} 2$, présentant initialement un PSA très élevé à $286 \mathrm{ng} / \mathrm{ml}$ avec un toucher rectal suspect T2c sur une prostate adénomateuse de 62g. L'IRM 7T biparamétrique de la pièce opératoire de prostatectomie radicale montre un envahissement très étendu de la partie droite de la glande depuis la base jusqu'à l'apex qui dans sa partie antérieure mesure $2,5 \times 1,5 \mathrm{~cm}$. L'interprétation initiale en aveugle de l'IRM 7T avait sous-évalué la tumeur car le volumineux envahissement caractéristique en hypoT2, hyperdiffusion avec une restriction ADC qui concerne plus de la moitié de la glande avait fait conclure à tort à une anomalie de signal non significative. On note après analyse histologique finale une parfaite superposition des zones tumorale sur toutes les séquences avec la coupe histologique correspondante. Le diagnostic la présence d'une tumeur pT3bNOMxR0 de score de Gleason $7(4+3)$ avec cette extension extracapsulaire aux vésicules séminales non représentées sur l'imagerie ; PSA indosable à 1 an. Cet exemple illustre à la fois la superposition parfaite en carte de géographie des lésions IRM 7T superposables avec celles de la coupe histologique finale. 

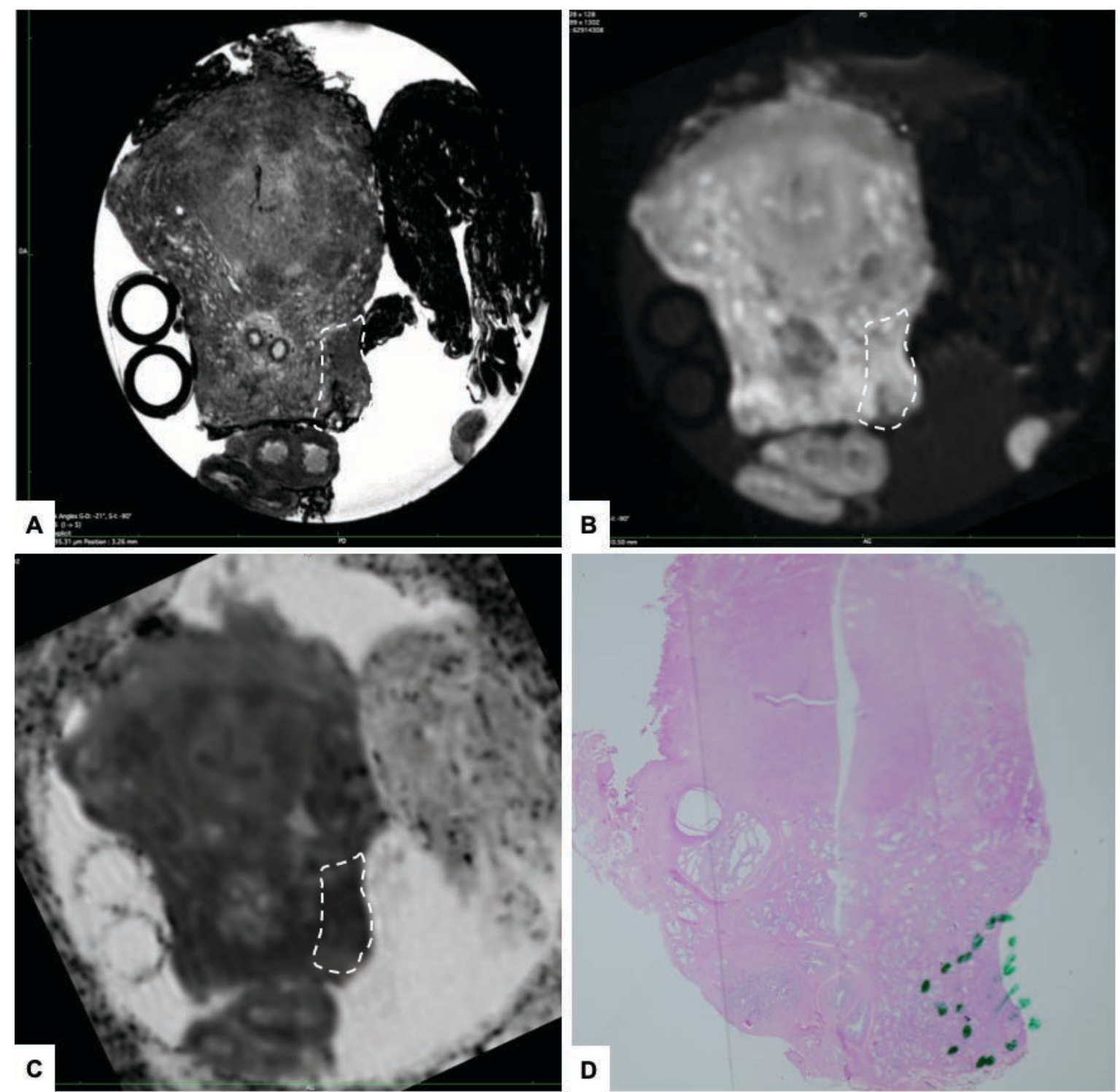

Figure 27 - Imagerie IRM 7T biparamétrique d'une volumineuse lésion prostatique de score de Gleason 7

Homme de 59 ans, obèse avec un IMC à $33 \mathrm{~kg} / \mathrm{m} 2$, sans antécédent, avec un PSA préopératoire de $3 \mathrm{ng} / \mathrm{ml}$ sur un toucher rectal normal T1c. II présentait un cancer de la prostate localisé pT2c de score de Gleason $7(3+4)$ infracentimétrique postéro-latérale gauche. Sur ce cas \#15, la corrélation était très élevée avec une superposition exacte du secteur suspecté par le relecteur avec la zone envahie par le cancer sur l'histologie finale. La lésion est en classé par le relecteur en hyposignal T2 avec un score de perceptibilité de $2 / 5$, non visible en diffusion et avec une restriction modérée en ADC, scoré $3 / 5$. 


\section{Sémiologie descriptive de lésions IRM 7T confirmées à I'histologie finale}

Les lésions de la zone périphérique postérieure. Les lésions de la zone périphérique postérieure comme celle de la Figure 28 sont identifiables sur les séquences anatomiques en T2W par l'association de plusieurs critères morphologiques caractéristiques. On notait la présence d'un hyposignal relatif (pas d'hypersignal) aux limites précises par rapport au tissu bénin de voisinage. Les tumeurs prenaient souvent une forme nodulaire à bord convexe avec un signal homogène contrastant nettement avec le tissu sain. La microstructure acineuse disparaissait au dépend d'une homogénéité de signal en plage soulignant la compacité du signal sans spot d'hypersignal tubuloacinaire.

La lésions de la zone de transition. L’hyper cellularité élevée de la zone de transition, volontiers remaniée par les processus adénomateux multinodulaires, diminue le coefficient de diffusion apparent de façon moins importante que ne l'entraîne la présence d'une lésion d'adénocarcinome. La Figure 29 rend compte d'une lésion de la zone de transition détectable par ses anomalies de signal en hypo T2W concordant en diffusion avec un hypersignal et une restriction significative de l'ADC sur la même zone. La diffusion, dans notre série, semble de moins apport que les autres séquences. L'asymétrie droite/gauche sur le pourtour nodulaire des lésions adénomateuse est très évocateur de la présence d'un processus expansif malin. La topographie lésionnelle superposable sur les différentes séquences, dépassant très nettement les limites de la capsule et surpassant les limites cerclées du liséré des nodules bénins, bien visible sur les séquences en T2W, constituent des signes non équivoques de lésions malignes. 
Le lésions du stroma fibromusculaire antérieur. Le SFMA est classiquement en hyposignal. Dans notre série, les lésions antérieures apparaissaient néanmoins avec un hyposignal légèrement profond que le tissu que le tissu sain. La Figure 30 rend compte de cette sémiologie. Les critères fonctionnels concordants étaient très informatifs. Notamment, la restriction significative de l'ADC et l'hypersignal en diffusion très marqué sur ces lésions renforçait l'évaluation diagnostique en faveur d'une forte probabilité lésionnelle. 


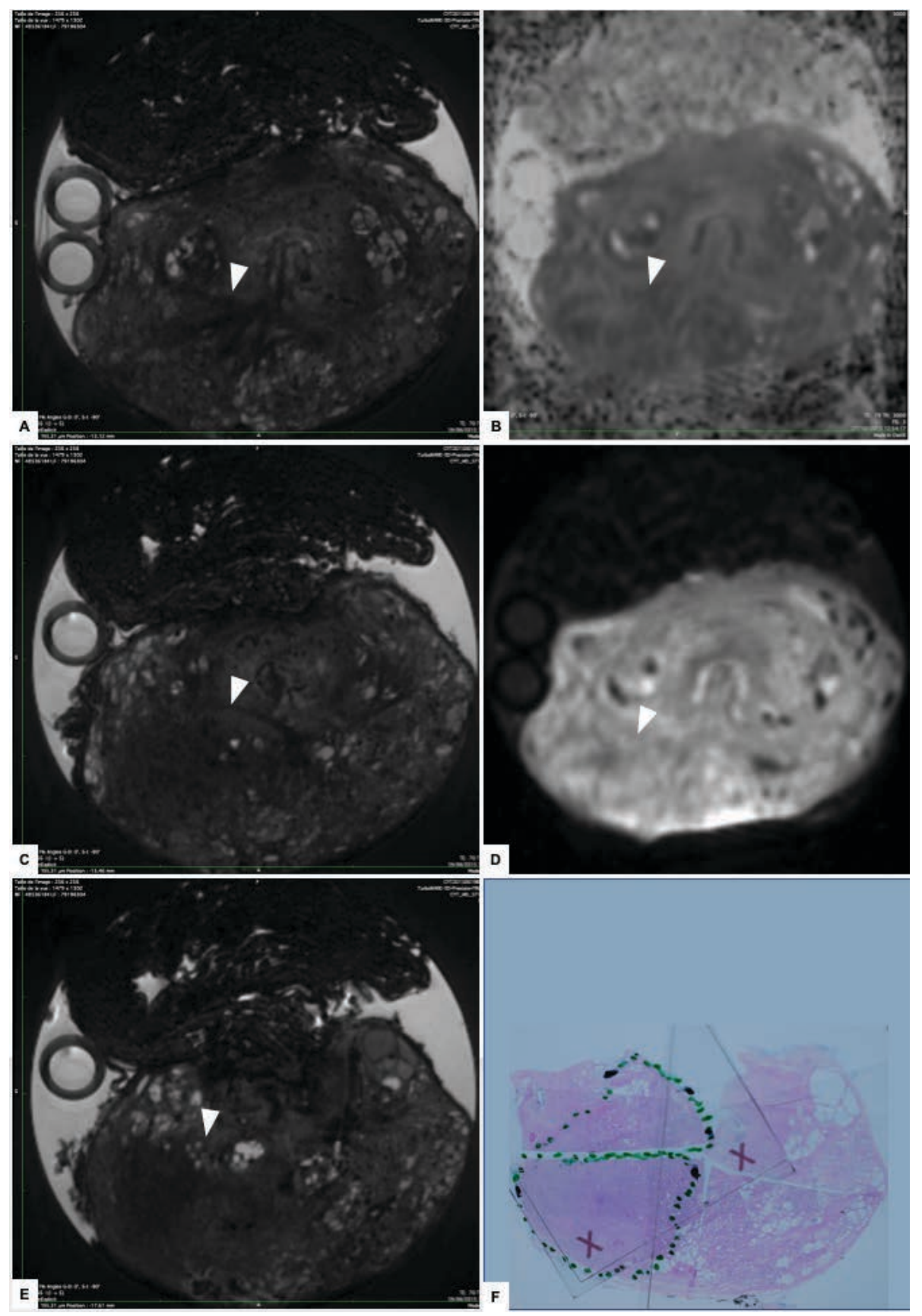

Figure 28 - Imagerie IRM 7T biparamétrique d'une lésion postérieure prostatique de score de Gleason 7

Homme de 60 ans avec un PSA initial à $4,8 \mathrm{ng} / \mathrm{ml}$, T1c, sur une prostate de petit volume de $33 \mathrm{~g}$. L'IRM 7T biparamétrique de la pièce opératoire de prostatectomie radicale montre une lésion de la zone de périphérique envahissant environ $10 \%$ de la prostate de $1,7 \mathrm{~cm}$ de grand axe. Elle est détectable et bien délimitée sur les 3 coupes successives en séquence T2 avec un franc hyposignal. La zone suspecte de tumeur est positive à l'analyse histologique finale qui confirme la présence d'un adénocarcinome pT2. Les autres séquences ne sont pas très informatives avec sur les coupes équivalentes une lésion en iso-signal. 

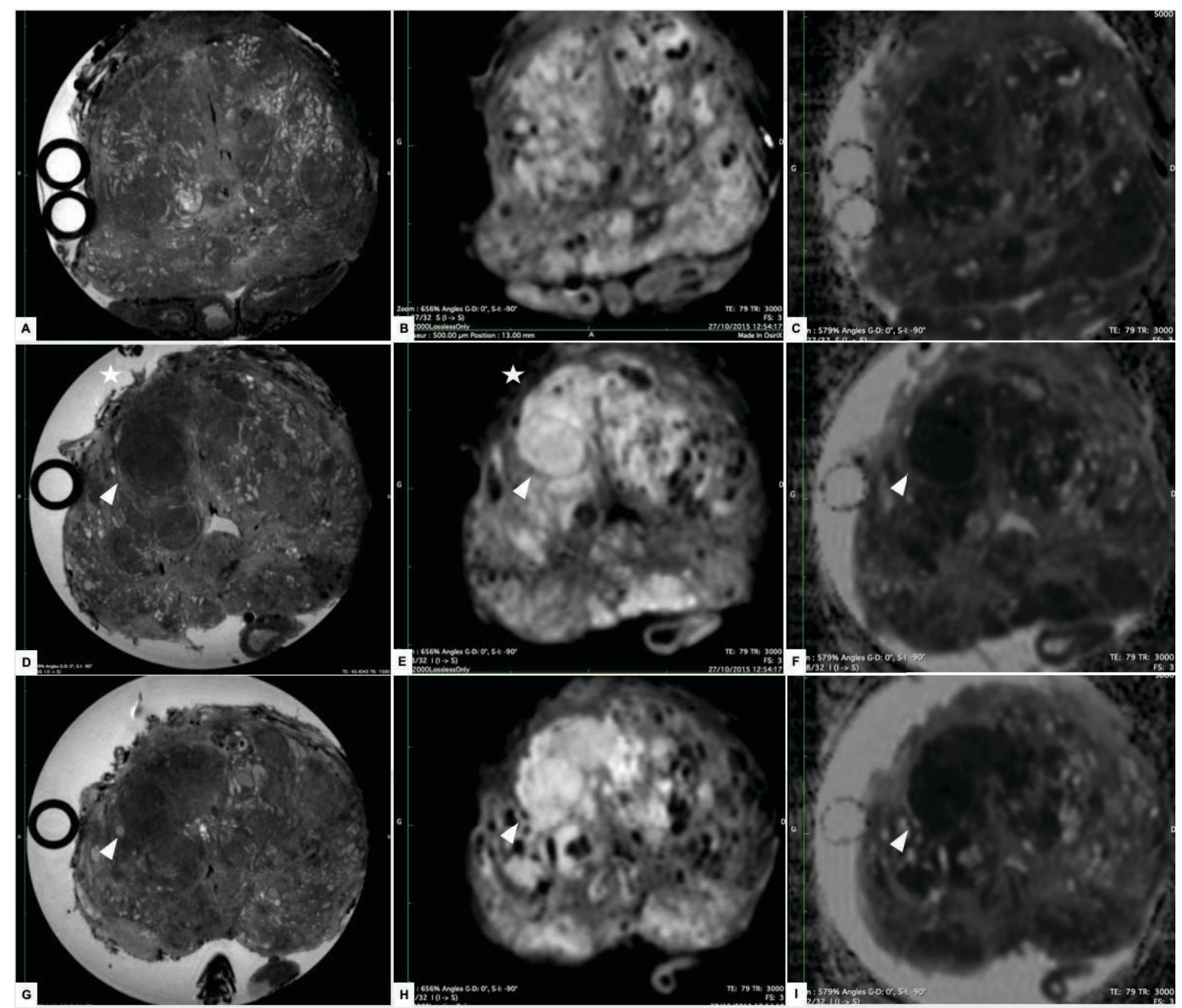

Figure 29 - Imagerie IRM 7T biparamétrique d'une lésion de zone de transition de score de Gleason 6

Homme de 79 ans avec un PSA initial à $6 \mathrm{ng} / \mathrm{ml}$, T1c, sur une prostate adénomateuse de 52g. L'IRM 7T biparamétrique de la pièce opératoire de prostatectomie radicale montre une lésion de la zone de transition de taille significative $1,5 \times 1,2 \mathrm{~cm}$. Elle est détectable et bien délimitée sur les 3 séquences avec un franc hyposignal en $\mathrm{T} 2 \mathrm{~W}$, un hypersignal très net en diffusion $(\mathrm{DWI})$ avec une cellularité élevée diminuant le coefficient de diffusion apparent (restriction de l'ADC). L'adénome multinodulaire antérieur perturbe peu l'interprétation, la lecture asymétrique droite/gauche à contours plus limité à droite avec une sémiologie concordante sur les 3 séquences rend le diagnostic plus aisé. La tumeur présente une extension extra-prostatique antérieure droite en marge négative (étoile blanche). L'analyse de la marge est bien contrôlée sur les images en diffusion. Le diagnostic est confirmé par l'analyse histologique final s'agissant d'une tumeur pT3aNOMxR0 de score de Gleason 6 avec cette extension extracapsulaire focale; PSA indosable à 1 an. Cet exemple illustre la sémiologie radiologique d'une lésion de faible risque de la zone de transition avec complémentarité des séquences nécessaire pour poser le diagnostic. 

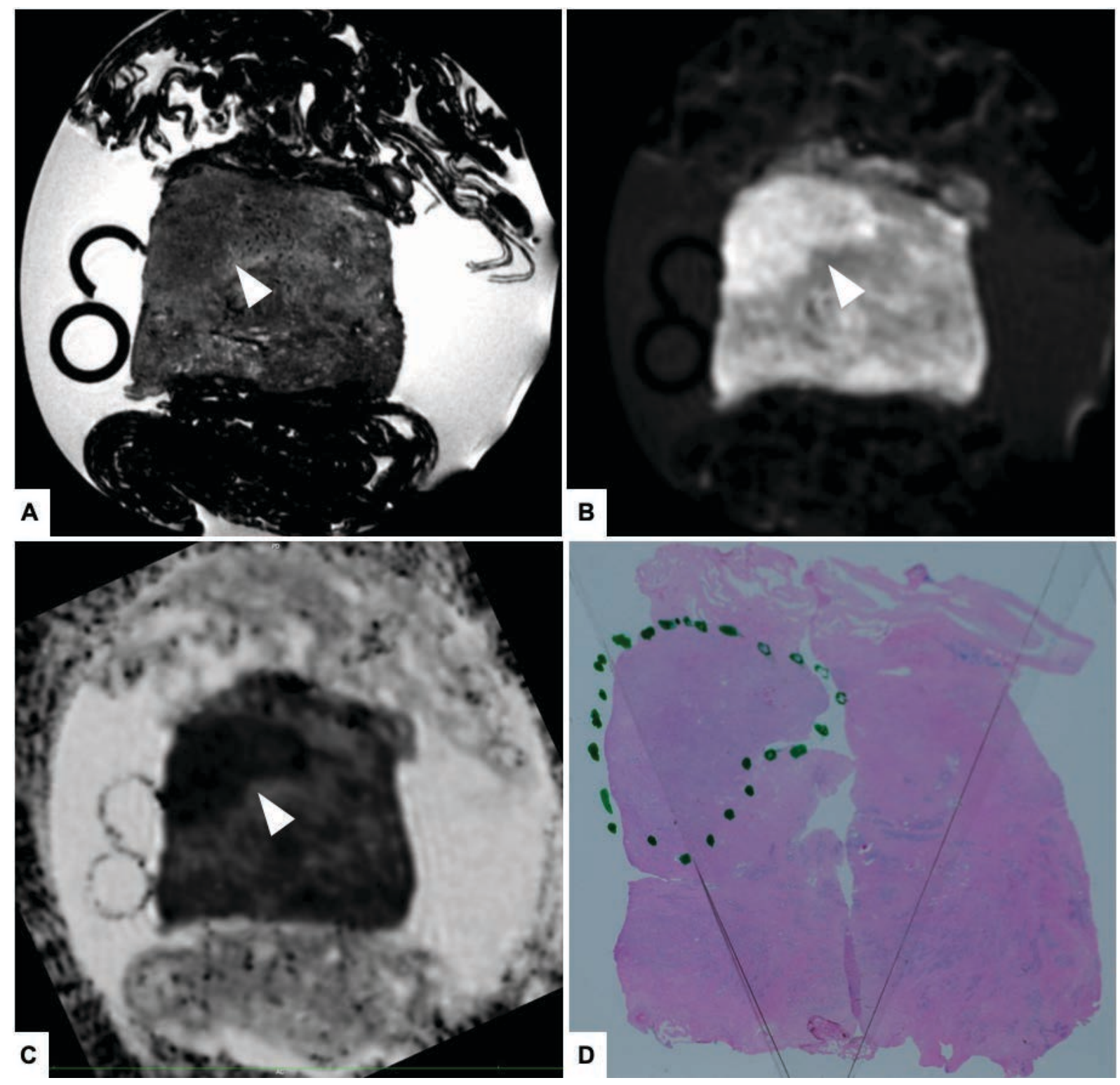

Figure 30 - Imagerie IRM 7T biparamétrique d'une lésion antérieure prostatique de score de Gleason 7

Homme de 49 ans avec un PSA initial à $14 \mathrm{ng} / \mathrm{ml}$, T1c, sur une prostate de taille normale de $24 \mathrm{~g}$. L'IRM 7T biparamétrique de la pièce opératoire de prostatectomie radicale montre une lésion de la zone antérieure et transitionnelle de l'apex. Elle mesurait $1,3 \mathrm{~cm}$ de grand axe. Elle est détectable et bien délimitée sur les 3 séquences avec un hyposignal en T2W, un hypersignal très net en diffusion (DWI) et une restriction importante de l'ADC. La topographie lésionnelle est en tout point superposable avec l'analyse histologique finale. Le diagnostic confirmait la présence d'une lésion adénocarcinomateuse pT3cNOMxR0 de score de Gleason $7(4+3)$. 


\section{k. Discussion}

A ce jour, I'IRM multiparamétrique utilisée en pratique clinique courante pour diagnostiquer le cancer de la prostate manque de puissance et rate $13 \%$ [10] de lésions significatives dans les meilleures séries. L'ensemble de notre travail consistait à développer des nouveaux moyens pour dépasser cette limite et augmenter les performances diagnostiques IRM. Parmi les mesures d'optimisation de performance de I'IRM, nous avions retenu de travailler à l'amélioration de la résolution spatiale. Notre étude s'attachait à évaluer le comportement d'une IRM 7T expérimentale en utilisant un modèle ex vivo d'imagerie de pièces fraîches de prostatectomies radicales pour cancer après exérèses chirurgicales robot-assistées réalisées dans un seul centre. Le RSB plus élevé de l'IRM 7T offrait la possibilité d'explorer plusieurs nouveaux mécanismes de contraste intéressants pour l'évaluation diagnostique du cancer de prostate. Suite à plusieurs optimisations de séquence, nous avons choisi de réaliser des séries d'imagerie biparamétriques en T2W, DWI et ADC.

Ces travaux s'appuyaient sur une première série d'analyses effectuées antérieurement sur des coupes d'échantillons de prostates humaines. Il avait alors étét établi que la résolution spatiale cible de $60 \times 60 \times 60 \mu \mathrm{m}^{3}$, obtenue selon certains paramétrages, permettait d'apporter un niveau d'information radiologique équivalent à celui obtenu en histologie avec un grossissement X2 nécessaire pour le diagnostic anatomo-pathologique du cancer de prostate. En transposant les paramétrages de nos premiers travaux sur une nouvelle plateforme d'IRM $7 \mathrm{~T}$, nous avons réalisé l'imagerie d'une nouvelle série de 18 spécimens complets. La qualité des images était appréciée par 2 relecteurs indépendants en aveugle. Les performances diagnostiques de l'IRM 7T étaient évalués par un radiologue expert pour le diagnostic du cancer de 
prostate. Les analyses ont permis de définir les bases d'une nouvelle radio-sémiologie en IRM 7T du tissu prostatique selon les zones d'étude conventionnelles et la nature du tissu bénin ou malin.

La qualité des images obtenues à des résolutions maximum en T2W de 195 X 195 X $195 \mu \mathrm{m}^{3}$ en 4 h30 environ a été jugée dans l'ensemble très bonne avec un score de qualité hautement concordant entre les 2 relecteurs. Notre méthodologie d'analyse statistique de concordance et de corrélation reposant sur le recours à la fois numérique et graphique de 2 séries de tests différents et concluant à un même niveau de résultats, a rendu d'autant plus robustes nos conclusions. La qualité obtenue à cette résolution d'image permettait notamment de distinguer tous les détails structurels tissulaires prostatique à une échelle infra-millimétrique, confirmant nos premiers travaux expérimentaux [48]. Cette précision de lecture était permise grâce à l'apport de signal complémentaire, conséquence des réglages d’optimisation de séquence et d'augmentation de l'aimant. Le fait qu'il a été noté qu'il y ait moins de corrélation dans la distribution des valeurs de faibles scores entre les deux relecteurs pourrait venir de la différence d'expérience importante qu'il existait entre le relecteur \#1 - urologue - et le relecteur \#2 - radiologue -. Cet écart pourrait aussi s'expliquer par le caractère novateur de la nouvelle sémiologie 7T, plus facilement appréhendable et interprétable par un relecteur expert.

Les performances diagnostiques obtenues n'atteignaient cependant pas dans notre série des valeurs supérieures à l'IRM classique de pratique clinique courante. Notamment, la VPN pour toutes les lésions et pour les lésions indexes étaient de 75 et $82 \%$ respectivement. Ces résultats étaient inférieurs aux prévisions espérées et à 
ce que laissait présager la qualité des imageries obtenues. L'absence d'imagerie de perfusion, habituellement recommandé pour l'analyse et non substituable, concourait sans doute à la diminution de la performance générale pour l'analyse de la prostate périphérique. Mais le déficit de performance s'expliquait également par l'augmentation importante de la résolution spatiale qui est perturbatrice puisqu'elle permettait de mieux analyser le contenu tumoral au détriment de la résolution en contraste. Ce frein à l'interprétation a déjà été constaté dans le passage des machines 1,5T à 3T. En effet de la même manière, l'amélioration rapportée de la qualité des images en 3T et encore plus en 7T (images plus fines, contraste excellent, séquences moins bruités) se faisait au prix d'une sensibilité plus importante aux artéfacts, notamment aérique (contrainte de notre modèle), et d'une augmentation du nombre d'anomalie de signal. Dès lors, les critères sémiologiques classiquement retenus ne correspondaient plus à l'ensemble des critères nécessaires d'analyser pour identifier et caractériser en particulier les zones suspectes de cancer.

La résolution spatiale impressionnante du $\mathrm{T} 2 \mathrm{~W}$ rendait parfois les tumeurs isointenses. Le diagnostic était fait sur l'impression visuelle de compacité ce qui induisait des erreurs d'interprétation de lecture par omission. Le RSB était amélioré et ne pouvait être mis en cause dans les problématiques d'interprétation. Nos résultats présentent une première série de constats et de fondamentaux pour lire une IRM 7T. De là, le dogme de l'hyposignal T2 systématique, notamment en zone périphérique, pourrait être remis en cause si nos résultats se confirmaient avec une plus grande série. La diffusion obtenue en IRM 7T semblait aussi assez décevante en soi car la plupart des lésions étaient peu visibles. La cartographie ADC, en revanche, était très contributive et nettement supérieure à la diffusion brute. 
Sur cette base, il serait nécessaire de réinventer une sémiologie d'imagerie haute résolution avec des outils de traitement différents notamment pour améliorer la résolution en contraste et analyser la texture des images. Classiquement en IRM, on s'intéresse au signal, puis aux catégories linéaires et radiaires. Dans la sémiologie conventionnelle d'analyse des lésions, on considère ces différents niveaux d'analyses par zone. Pour la zone périphérique par exemple, on recherche principalement la concordance entre des critères morphologiques et des critères fonctionnels en imagerie multiparamétrique. En cas de concordance, la suspicion lésionnelle est forte et inversement. Selon nos résultats, les critères morphologiques d'identification du cancer de prostate semblaient prendre une place importante parmi les outils diagnostiques. En particulier, la compacité du signal et la forme nodulaire étaient des signes que l'on retrouvait fréquemment dans nos analyses lésionnelles en cas de cancer confirmé.

La faiblesse du nombre d'échantillons fragilisait les résultats et leur interprétation. La puissance de l'analyse dans notre étude est trop petite pour émettre une conclusion à ce stade. Mais I'IRM semblait pour autant révéler de bonnes performances, spécialement en termes de spécificité. A noter que les résultats pour les lésions indexes étaient relativement comparables. Les corrélations kappa étaient aussi difficiles à interpréter en raison du nombre restreint de sujets. La disparité entre les quadrants et les cibles (totales ou indexes) ainsi que les larges intervalles de confiance, en témoignaient. 
La corrélation radio-histologique avait été réalisée en utilisant seulement une grille de comparaison par cadrant selon 6 secteurs (base, médian, apex à droite et à gauche). Le schéma de partitionnement de la prostate recommandé par la conférence de consensus PREDICT (Londres, 2010) [55], en 27 secteurs, ne pouvait pas être appliqué à l'analyse des lésions histologiques. Cette cartographie est pourtant bien celle qui fait référence aujourd'hui en soins courant. Mais l'interprétation bidimensionnelle par coupe, selon ces 27 secteurs, des lésions histologiques identifiées est complexe et source fréquente de biais rapportés dans les études corrélatives. Nous n'avons pas pu dépasser cette contrainte. Nous avons tenté de limiter l'écueil de transposition bidimensionnelle des lésions histologiques par l'usage d'une technique de report sur coupe dont le nombre était laissé à la discrétion de l'anatomopathologiste de référence de notre étude en fonction du volume de prostate qu'il appréciait. Ce système demeurait imparfait et sans doute source de biais d'analyse dans les tests de corrélation et le calcul de performance. Nous n'avons pas non plus analysé la différence diagnostique entre la partie postérieure et antérieure. 


\section{Perspectives de développement et d'applications cliniques}

Ce travail a porté sur des modèles expérimentaux d'imagerie ex vivo sur tissus prostatiques et glandes entières en IRM 7T pour dépasser la résolution spatiale de la pratique clinique courante, et améliorer la détection tumorale. Ces résultats serviront de base au développement de diverses applications et à la translation future de l'imagerie actuelle vers l'IRM 7T in vivo chez l'homme.

Des perspectives immédiates s'ouvrent d'abord sur des possibilités d'exploitation de notre technique en imagerie haute résolution. L'imagerie de la glande entière avant préparation pourrait constituer une étape préalable d'analyse et d'identification tissulaire pour l'anatomo-pathologiste. L'IRM 7T pourrait aider à diagnostiquer et à caractériser le cancer de prostate, mais aussi à fournir des informations complémentaires au-delà des possibilités actuelles. Notamment, l'analyse tridimensionnelle par imagerie IRM 7T morphologique pré-étude macro, permettrait la caractérisation d'une marge positive chirurgicale en apportant une analyse lésionnelle avant la coupe histologique avec une étude plus précise de la longueur de la marge.

En soins courants, la transposition de nos paramètres de séquences sur des plateformes IRM 7T existantes et adaptées à l'imagerie humaine in vivo - de type Magnetom 7T (Siemens Healthcare, Erlangen, Germany) - nécessite plusieurs tests et aménagements préalables de nos protocoles. Des premiers résultats sur témoins sains ont été publiés [56] sans qu'ils puissent démontrer, à ce stade, d'un niveau de qualité équivalent au nôtre du fait, sans doute, d'une dégradation importante du RSB. La situation anatomique pelvienne de la prostate représente une contrainte qui conduit à cette perte de signal. Des adaptations sont donc nécessaires en considérant que 
temps d'acquisition et haute résolution d'image sont très interdépendants. En pratique clinique, un compromis devra être trouvé entre les deux pour optimiser les paramètres de séquences IRM 7T chez l'homme.

Mais compte tenu de nos résultats et de la rapidité d'avancement des techniques d'imagerie radiologique, il est raisonnable de supposer que les images IRM 7T de patients in vivo approcheront la frontière de la résolution proche de l'histologique à l'avenir. Les radiologues évalueront des formes de contraste d'images différentes de celles actuellement acceptées pour interpréter des images à haute résolution de prostate comme celles que nous avons réalisées. Une nouvelle grille de lecture et une sémiologie IRM 7T doivent être décrites pour permettre cette analyse de niveaux de signal riches de millions de données, non perceptibles par l'œil humain seul.

Pour écrire et valider cette sémiologie IRM 7T, il convient de poursuivre l'étude corrélative radio-histologique des anomalies de signal avec l'histologie finale en utilisant exclusivement des coupes histologiques numérisées selon une épaisseur et un plan identique à ceux de l'imagerie. Le mieux serait de pouvoir comparer images IRM 7T avec des coupes correspondantes numérisées pour améliorer la comparabilité et constituer ainsi un ensemble de données normalisées de référence, utilisable pour entraîner une recherche algorithmique qui constituerait une voie de développement majeure de l'IRM 7T. Des programmes d'assistance diagnostique par intelligence artificielle (IA) pourraient alors être envisagés avec cette nouvelle imagerie et cette néo-sémiologie IRM 7T. L'IA et l'apprentissage automatique (machine learning (ML)) qui progressent dans différents domaines d'applications médicales et particulièrement en imagerie [57], pourraient être appliqués à nos résultats. Des progrès récents dans 
I'IA / ML sont significatifs dans le secteur de l'analyse d'images à tous les niveaux, de la microscopie à la radiologie depuis la publication de la classification ImagNet [5859]. Nos résultats, qui témoignent de la possibilité d'obtenir une imagerie haute résolution avec un niveau de différence de signal à l'échelle du millimètre, concourent au développement de l'analyse d'imagerie IRM assistée par IA / ML pour exploiter son potentiel. 


\section{Conclusions}

La qualité d'une image IRM dépend de plusieurs facteurs dont la résolution spatiale, le contraste, le bruit et les artefacts. Notre série de travaux consistait à développer plusieurs modèles expérimentaux pour tester différents paramètres d'optimisation de séquence IRM pour augmenter la résolution spatiale. Grâce à la réduction de la taille des voxels en jouant sur la dimension de la matrice, le champ visuel et l'épaisseur de la coupe, nous avons obtenu des résolutions de $60 \times 60 \times 90 \mu \mathrm{m}^{3}$ et $130 \times 130 \times 195$ $\mu \mathrm{m}^{3}$, sur des coupes et glandes entières de prostatectomies fraîches, respectivement. Ces niveaux de résolution étaient comparables à ceux obtenus en analyse histologique finale de pièces opératoires avec un grossissement X2. Ils permettaient notamment de distinguer des éléments de la microarchitecture prostatique (canaux, vaisseaux...). La précision d'analyse des micro-structures tissulaires étaient renforcée par l'amélioration associée du contraste des images obtenues. Les optimisations de séquences réalisées rendaient l'étude micro-anatomique des tissus plus aisés, notamment en T2W, grâce à la perception de différents niveaux de signal entre les structures tissulaires à une échelle microscopique. En fin de compte, la qualité des images réalisées a permis de caractériser de façon reproductible, au moyen de différentes séquences d'IRM bi-paramétriques, la présence de cancer de prostate sur les modèles d'étude. L'ambition de nos séries d'analyses était d'apporter, outre les preuves de leur faisabilité, les fondements sémiologiques radiologique d'une imagerie haute résolution et d'identifier les paramètres de protocole IRM à modifier pour plus de performance en résolution spatiale d'imagerie clinique. 


\section{Références}

1. Hricak H, Choyke PL, Eberhardt SC, Leibel SA, Scardino PT. Imaging prostate cancer: a multidisciplinary perspective. Radiology 2007, 243: 28-53.

2. Cornud F, Villers A, Mongiat-Artus $P$, Rebillard $X$, Soulie $M$, les membres du sous-comité « Prostate » du CCAFU. [Magnetic resonance imaging and prostate cancer]. Prog Urol. nov 2008;18(10):621-33.

3. Barentsz JO, Richenberg J, Clements R, Choyke P, Verma S, Villeirs G, et al. ESUR prostate MR guidelines 2012. European Journal of Radiology, 2012, 22: 746-757.

4. Ahmed HU, Kirkham A, Arya M, Illing R, Freeman A, Allen C, et al. Is it time to consider a role for MRI before prostate biopsy? Nature Reviews, Clinical Oncology 2009, 6: 1997- 206.

5. Turkbey B, Mani H, Shah V, Rastinehad AR, Bernardo M, Pohida T, et al. Multiparametric 3T prostate magnetic resonance imaging to detect cancer: histopathological correlation using prostatectomy specimens processed in customized magnetic resonance imaging based molds. J Urol. nov 2011;186(5):1818-24.

6. Cornud F, Flam T, Chauveinc L, Hamida K, Chrétien Y, Vieillefond A, et al. Extraprostatic spread of clinically localized prostate cancer: factors predictive of pT3 tumor and of positive endorectal MR imaging examination results. Radiology. juill 2002;224(1):203-10.

7. Rozet F, Hennequin C, Beauval J-B, Beuzeboc P, Cormier L, Fromont-Hankard G, et al. Recommandations françaises du Comité de Cancérologie de l'AFU - Actualisation 2018-2020 : cancer de la prostate. Progrès en Urologie. 2018 Nov 1;28(12, Supplement):S79-130.

8. Sciarra A, Barentsz J, Bjartell A, Eastham J, Hricak H, Panebianco V, et al. Advances in magnetic resonance imaging: how they are changing the management of prostate cancer. European Urology, 2011, 59: 962-977.

9. Rouvière $\mathrm{O}$, Hartman RP, Lyonnet D. Prostate MR imaging at high-field strength: evolution or revolution? Eur Radiol. févr 2006;16(2):276-84.

10. Baco E, Rud E, Eri LM, Moen G, Vlatkovic L, Svindland A, et al. A Randomized Controlled Trial To Assess and Compare the Outcomes of Two-core Prostate Biopsy Guided by Fused Magnetic Resonance and Transrectal Ultrasound Images and Traditional 12-core Systematic Biopsy. Eur Urol. janv 2016;69(1):149-56.

11. Barret E, Turkbey B, Puech P, Durand M, Panebianco V, Fütterer JJ, et al. Update on the ICUD-SIU consultation on multi-parametric magnetic resonance imaging in localised prostate cancer. World J Urol. 12 juill 2018;

12. Jéhannin-Ligier K, Dantony E, Bossard N, Molinié F, Defossez G, Daubisse-Marliac L, Delafosse P, Remontet L, Uhry Z. Projection de l'incidence et de la mortalité par cancer en France métropolitaine en 2017. Rapport technique. Saint-Maurice : Santé publique France, 2017. 80 p

13. Chaumard-Billotey et al. 17th ECCO, 38th ESMO, 32th ESTRO, Amsterdam, 27 September- 1 October 2013; poster 417

14. Mouraviev V, Villers A, Bostwick DG, Wheeler TM, Montironi R, Polascik TJ. Understanding the pathological features of focality, grade and tumour volume of early-stage prostate cancer as a foundation for parenchyma-sparing prostate cancer therapies: active surveillance and focal targeted therapy. BJU Int. 2011 Oct;108(7):1074-85.

15. Postma R, de Vries SH, Roobol MJ, Wildhagen MF, Schröder FH, van der Kwast TH. Incidence and follow-up of patients with focal prostate carcinoma in 2 screening rounds after an interval of 4 years. Cancer. 2005 Feb 15;103(4):708-16. 
16. Stamey TA, Freiha FS, McNeal JE, Redwine EA, Whittemore AS, Schmid HP. Localized prostate cancer. Relationship of tumor volume to clinical significance for treatment of prostate cancer. Cancer. 1993 Feb 1;71(3 Suppl):933-8.

17. Epstein JI, Walsh PC, Carmichael M, Brendler CB. Pathologic and clinical findings to predict tumor extent of nonpalpable (stage T1c) prostate cancer. JAMA. 1994 Feb 2;271(5):368-74.

18. Harnden P, Naylor B, Shelley MD, Clements H, Coles B, Mason MD. The clinical management of patients with a small volume of prostatic cancer on biopsy: what are the risks of progression? A systematic review and meta-analysis. Cancer. 2008 Mar 1;112(5):971-81.

19. Schröder FH, Hugosson J, Roobol MJ, Tammela TLJ, Ciatto S, Nelen V, et al. Prostate-cancer mortality at 11 years of follow-up. N Engl J Med. 15 mars 2012;366(11):981-90.

20. Andriole GL, Crawford ED, Grubb RL 3rd, Buys SS, Chia D, Church TR, et al. Prostate cancer screening in the randomized Prostate, Lung, Colorectal, and Ovarian Cancer Screening Trial: mortality results after 13 years of follow-up. J Natl Cancer Inst. 18 janv 2012;104(2):125-32.

21. Hugosson J, Carlsson S, Aus G, Bergdahl S, Khatami A, Lodding $P$, et al. Mortality results from the Göteborg randomised population-based prostate-cancer screening trial. Lancet Oncol. août 2010;11(8):725-32.

22. Ahmed HU, El-Shater Bosaily A, Brown LC, Gabe R, Kaplan R, Parmar MK, et al. Diagnostic accuracy of multi-parametric MRI and TRUS biopsy in prostate cancer (PROMIS): a paired validating confirmatory study. Lancet. 25 2017;389(10071):815-22.

23. Walz J. The «PROMIS » of Magnetic Resonance Imaging Cost Effectiveness in Prostate Cancer Diagnosis? Eur Urol. janv 2018;73(1):31-2.

24. Puech $P$, Villers A, Ouzzane A et al (2014) Prostate cancer: diagnosis, parametric imaging and standardized report. Diagn Interv Imaging 95(7-8):743-752

25. Weinreb JC, Barentsz JO, Choyke PL et al (2016) PI-RADS prostate imaging-reporting and data system: 2015, Version 2. Eur Urol 69(1):16-40

26. Villers A, Lemaitre L, Haffner J, Puech P. Current status of MRI for the diagnosis, staging and prognosis of prostate cancer: implications for focal therapy and active surveillance. Curr Opin Urol. mai 2009;19(3):274-82.

27. Villers A, Puech P, Mouton D, Leroy X, Ballereau C, Lemaitre L. Dynamic contrast enhanced, pelvic phased array magnetic resonance imaging of localized prostate cancer for predicting tumor volume: correlation with radical prostatectomy findings. J Urol. déc 2006;176(6 Pt 1):2432-7.

28. Yoo S, Kim JK, Jeong IG. Multiparametric magnetic resonance imaging for prostate cancer: A review and update for urologists. Korean J Urol. juill 2015;56(7):487-97.

29. Tay KJ, Gupta RT, Brown AF, Silverman RK, Polascik TJ. Defining the Incremental Utility of Prostate Multiparametric Magnetic Resonance Imaging at Standard and Specialized Read in Predicting Extracapsular Extension of Prostate Cancer. Eur Urol. 2016;70(2):211-3.

30. Kobus T, Hambrock T, Hulsbergen-van de Kaa CA, Wright AJ, Barentsz JO, Heerschap A, et al. In vivo assessment of prostate cancer aggressiveness using magnetic resonance spectroscopic imaging at 3 T with an endorectal coil. Eur Urol. nov 2011;60(5):1074-80.

31. Hambrock T, Somford DM, Huisman HJ, van Oort IM, Witjes JA, Hulsbergen-van de Kaa CA, et al. Relationship between apparent diffusion coefficients at 3.0-T MR imaging and Gleason grade in peripheral zone prostate cancer. Radiology. mai 2011;259(2):453-61. 
32. Moore CM, Robertson NL, Arsanious N, Middleton T, Villers A, Klotz L, et al. Image-guided prostate biopsy using magnetic resonance imaging-derived targets: a systematic review. Eur Urol. janv 2013;63(1):125-40.

33. Guichard G, Larré S, Gallina A, Lazar A, Faucon H, Chemama S, et al. Extended 21-sample needle biopsy protocol for diagnosis of prostate cancer in 1000 consecutive patients. Eur Urol. août 2007;52(2):430-5.

34. Pokorny MR, de Rooij M, Duncan E, Schröder FH, Parkinson R, Barentsz JO, et al. Prospective study of diagnostic accuracy comparing prostate cancer detection by transrectal ultrasound-guided biopsy versus magnetic resonance (MR) imaging with subsequent MR-guided biopsy in men without previous prostate biopsies. Eur Urol. juill 2014;66(1):22-9.

35. Ahmed HU, Freeman A, Kirkham A, Sahu M, Scott R, Allen C, et al. Focal therapy for localized prostate cancer: a phase I/II trial. J Urol. avr 2011;185(4):1246-54.

36. Donaldson IA, Alonzi R, Barratt D, Barret E, Berge V, Bott S, et al. Focal therapy: patients, interventions, and outcomes--a report from a consensus meeting. Eur Urol. avr 2015;67(4):771-7.

37. Marshall S, Taneja S. Focal therapy for prostate cancer: The current status. Prostate Int. juin 2015;3(2):35-41.

38. Marliere F, Puech P, Benkirane A, Villers A, Lemaitre L, Leroy X, et al. The role of MRI-targeted and confirmatory biopsies for cancer upstaging at selection in patients considered for active surveillance for clinically low-risk prostate cancer. World J Urol. août 2014;32(4):951-8.

39. Van den Bergh RCN, Ahmed HU, Bangma CH, Cooperberg MR, Villers A, Parker CC. Novel tools to improve patient selection and monitoring on active surveillance for low-risk prostate cancer: a systematic review. Eur Urol. juin 2014;65(6):1023-31.

40. Ouzzane A, Renard-Penna R, Marliere F, Mozer P, Olivier J, Barkatz J, et al. Magnetic Resonance Imaging Targeted Biopsy Improves Selection of Patients Considered for Active Surveillance for Clinically Low Risk Prostate Cancer Based on Systematic Biopsies. J Urol. août 2015;194(2):350-6.

41. Léautaud A, Marcus C, Ben Salem D, Bouché O, Graesslin O, Hoeffel C. [Pelvic MRI at 3.0 Tesla]. J Radiol. mars 2009;90(3 Pt 1):277-86.

42. Bath KG, Voss HU, Jing D, Anderson S, Hempstead B, Lee FS, et al. Quantitative intact specimen magnetic resonance microscopy at 3.0 Tesla. Magnetic Resonance Imaging 2009, 27: 672-680.

43. Zhan Y, Feldman M, Tomaszewski J, Davatzikos C, Shen D. Registering histological and MR images of prostate for image-based cancer detection. Med Image Comput Comput Assist Interv 2006;9:620-8.

44. Fan X, Haney CR, Agrawal G, Pelizzari CA, Antic T, Eggener SE, et al. High-resolution MRI of excised human prostate specimens acquired with 9.4T in detection and identification of cancers: validation of a technique. Journal of Magnetic Resonance Imaging 2011, 34:956-961.

45. Bratan F, Niaf E, Melodelima $C$ et al. Influence of imaging and histological factors on prostate cancer detection and localisation on multiparametric MRI: a prospective study. Eur Radiol 2013; 23: 2019-29

46. Anwar M, Westphalen AC, Jung AJ et al. Role of endorectal MR imaging and MR spectroscopic imaging in defining treatable intraprostatic tumor foci in prostate cancer: quantitative analysis of imaging contour compared to whole-mount histopathology. Radiother Oncol 2014; 110: 303-8

47. Uribe CF, Jones EC, Chang SD, Goldenberg SL, Reinsberg SA, Kozlowski P. In vivo 3T and ex vivo 7T diffusion tensor imaging of prostate cancer: correlation with histology. Magn Reson Imaging 2015; 33: $577-83$ 
48. Durand M, Robinson BD, Aronowitz E, Tewari AK, Ballon DJ. High-resolution magnetic resonance imaging of prostatectomy specimens: a promising tool for virtual histology. Eur Urol. nov 2012;62(5):938-40.

49. Hou MF, Huang TJ, Liu GC. The diagnostic value of galactography in patients with nipple discharge. Clin Imaging. avr 2001;25(2):75-81

50. Hoult DI, Phil D. Sensitivity and power deposition in a high-field imaging experiment. J Magn Reson Imaging. juill 2000;12(1):46-67.

51. Srivastava A, Chopra S, Pham A, Sooriakumaran P, Durand M, Chughtai B, et al. Effect of a Riskstratified Grade of Nerve-sparing Technique on Early Return of Continence After Robot-assisted Laparoscopic Radical Prostatectomy. European Urology. 1 mars 2013;63(3):438-44.

52. Dickinson L, Ahmed HU, Allen C, Barentsz JO, Carey B, Futterer JJ, et al. Magnetic resonance imaging for the detection, localisation, and characterisation of prostate cancer: recommendations from a European consensus meeting. Eur Urol. 2011 Apr;59(4):477-94.

53. Ranganathan $P$, Pramesh CS, Aggarwal R. Common pitfalls in statistical analysis: Measures of agreement. Perspect Clin Res. déc 2017;8(4):187-91.

54. Tewari AK, Srivastava A, Huang MW, Robinson BD, Shevchuk MM, Durand M, et al. Anatomical grades of nerve sparing: a risk-stratified approach to neural-hammock sparing during robot-assisted radical prostatectomy (RARP). BJU Int. 2011 Sep;108(6 Pt 2):984-92.

55. Dickinson L, Ahmed HU, Allen C, Barentsz JO, Carey B, Futterer JJ, et al. Magnetic resonance imaging for the detection, localisation, and characterisation of prostate cancer: recommendations from a European consensus meeting. Eur Urol. 2011 Apr;59(4):477-94.

56. Maas MC, Vos EK, Lagemaat MW, Bitz AK, Orzada S, Kobus T, et al. Feasibility of T2 -weighted turbo spin echo imaging of the human prostate at 7 tesla. Magn Reson Med. 2014 May;71(5):1711-9.

57. Fogel AL, Kvedar JC. Artificial intelligence powers digital medicine. npj Digital Medicine. 14 mars 2018;1(1):5.

58. Krizhevsky, I. Sutskever, and G. E. Hinton, "ImageNet classification with deep convolutional neural networks," in Proc. Advances Neural Information Processing Systems, 2012, pp. 1097-1105.

59. Al diagnostics need attention. Nature. 15 2018;555(7696):285. 


\section{Articles publiés dans le cadre de la thèse}

Durand M, Jain M, Robinson B, Aronowitz E, El Douahy Y, Leung R, et al. Magnetic resonance microscopy may enable distinction between normal histomorphological features and prostate cancer in the resected prostate gland. BJU Int. 2017;119(3):414-23.

Barret E, Durand M, éditeurs. Technical Aspects of Focal Therapy in Localized Prostate Cancer [Internet]. Paris: Springer-Verlag; 2015. Disponible sur: //www.springer.com/us/book/9782817804835

Rastinehad AR, Durand M. A comparison of magnetic resonance imaging and ultrasonography (MRI/US)-fusion guided prostate biopsy devices: too many uncontrolled variables. BJU Int. avr 2016;117(4):548-9.

Barret E, Turkbey B, Puech P, Durand M, Panebianco V, Fütterer JJ, et al. Update on the ICUD-SIU consultation on multi-parametric magnetic resonance imaging in localised prostate cancer. World J Urol. 12 juill 2018. 